\title{
MUON COLLIDER TASK FORCE REPORT
}

C.Ankenbrandt, Y.Alexahin, V.Balbekov, E.Barzi, C.Bhat, D.Brommelsiek, A.Bross, A.Burov, A.Drozhdin, D.Finley, S.Geer, N.Gelfand, E.Gianfelice-Wendt, M.Hu, A.Jansson, C.Johnstone, J.Johnstone, Vl.Kashikhin, V.Kashikhin, M.Lamm, V.Lebedev, N.Mokhov, C.Moore, A.Moretti, D.Neuffer, K.-Y.Ng, M.Popovic, I.Rakhno, V.Shiltsev, P.Spentsouris, A.Striganov, A.Tollestrup, A.Valishev, A.Van Ginneken, K.Yonehara, C.Yoshikawa, A. Zlobin

FNAL

J.Norem

$A N L$

J.S.Berg, J.C.Gallardo, R.Gupta, H.Kirk, R.Palmer, R.Fernow, P.Wanderer $B N L$

A.Bogacz, Y.-C.Chao, Y.Derbenev, R.A.Rimmer

$J L A B$

G.Sabbi, P.Ferracin, S.Caspi, M.Zisman

$L B N L$

R.Abrams, K.Beard, R.P.Johnson, M.A.Cummings, S.A.Kahn, S.Korenev, D.Newsham, T.J.Roberts

Muons Inc.

D.B.Cline, Y.Fukui , A.Garren

UCLA

G.Hanson, A.Klier

UC Riverside

L.M.Cremaldi, D.J.Summers

University of Mississippi

\begin{abstract}
A summary is given of results from the first year of Muon Collider Task Force activities, together with a description of an updated longer-term R\&D plan. In particular, this report summarizes Muon Collider ring and ionization cooling channel design and simulation studies, progress towards testing a high-pressure RF cavity in a beam at the Muon Test Area, Helical Cooling Channel design and simulation studies, progress towards a fourcoil helical solenoid test, design considerations for a 6D cooling experiment, and High Temperature Superconductor magnet and conductor studies for a high field solenoid at the end of a Muon Collider cooling channel.
\end{abstract}




\section{Executive Summary}

Muon Colliders offer a possible long term path to lepton-lepton collisions at center-ofmass energies $\sqrt{s}_{\mathrm{s}} \geq 1 \mathrm{TeV}$. In October 2006 the Muon Collider Task Force (MCTF) proposed a program of advanced accelerator $R \& D$ aimed at developing the Muon Collider concept. The proposed R\&D program was motivated by progress on Muon Collider design in general, and in particular, by new ideas that have emerged on muon cooling channel design. The scope of the proposed MCTF R\&D program includes muon collider design studies, helical cooling channel design and simulation, high temperature superconducting solenoid studies, an experimental program using beams to test cooling channel RF cavities and a 6D cooling demonstration channel. The first year of MCTF activities are summarized in this report together with a brief description of the anticipated FY08 R\&D activities.

Muon Collider Design Studies: An important goal for the MCTF activity is, within the next two to three years, to establish one or more realizable muon collider parameter set(s), and hence identify the performance goals for the muon cooling channel. Past physics studies have shown that a Muon Collider with $\sqrt{\mathrm{s}}_{\mathrm{s}}=1 \mathrm{TeV}$ to a few TeV requires a minimum luminosity of $\sim 10^{34} \mathrm{~cm}^{-2} \mathrm{~s}^{-1}$ to probe with adequate sensitivity the physics beyond the Standard Model. Two design strategies are being investigated to achieve this luminosity. In the "high-emittance" strategy the muons of each sign are packaged in the minimum number of bunches (one per cycle) and the emittance goal corresponds to the beam-beam limit. In the "low-emittance" strategy the muons are packaged into many bunches with much lower transverse emittances. To establish the feasibility of the highand/or low-emittance schemes it is necessary to establish the existence of corresponding Collider Lattice designs that assume realistic parameters for magnets, rf, etc. Ring studies this year explored various lattices, paying attention to chromatic correction and momentum acceptance. The designs for the high-emittance lattice are close to achieving the desired momentum acceptance and dynamic aperture, although further studies are needed. However, new ideas are needed to achieve a ring design that corresponds to the low emittance collider scheme.

Cooling Channel Design and Simulation: In addition to different collider design strategies (low- or high-emittance), different cooling channel technologies are also being explored. Ionization cooling reduces the beams transverse phase space. To achieve 6D cooling requires also mixing the longitudinal and transverse degrees of freedom, which can be achieved using a helical arrangement of solenoids. Two such schemes are being pursued. The "Guggenheim" scheme has been developed over the last couple of years by the NFMCC. Its proponents are also members of the MCTF, and are continuing to further develop the Guggenheim design and understand its performance and limitations. The newer Helical Cooling Channel (HCC) scheme was proposed by members of Muons Inc. Since it is newer, the HCC design studies are less well advanced, and have been a focus of attention during the first year of MCTF activities. During this year HCC simulation studies have begun to explore various ways of integrating RF cavities into the channel, and also begun to explore the design of a high-field HCC section at the end of the channel which enables lower emittances to be achieved. The results of these studies are promising and, although many details still need to be understood, both HCC and Guggenheim channels could in principal be used to achieve the emittances required for 
the "high-emittance" Muon Collider scheme. The "low emittance" scheme will require something extra, beyond either an HCC or a Guggenheim channel. The additional cooling idea that is being explored has been called Phase Ionization Cooling (PIC). Although there has progress on developing the PIC concept it is not yet sufficiently understood to enable design studies to begin based on tracking simulations of a PIC channel. Further PIC studies must be pursued in the coming year.

High Pressure RF Studies: The present designs for the phase rotation and cooling channels require high gradient RF cavities to operate in magnetic fields of up to a few Tesla. MUCOOL R\&D has shown that when operated in a magnetic field vacuum pillbox cavities breakdown at lower gradients. The continuing MUCOOL R\&D program is exploring ways to mitigate this effect, using alternative materials, surface treatments, and magnetic geometries. Previous measurements with open cell vacuum cavities showed no significant performance degradation in a magnetic field, however open cell cavities require a doubling of the required peak RF power and a corresponding increase in cost. Muons Inc. has proposed an alternative and potentially cost effective solution using cavities filled with high pressure gas to both suppress breakdown and provide the energy loss absorber. A High Pressure RF (HPRF) test cell has been shown to operate in a magnetic field with no appreciable reduction in the achievable RF gradient. However, these tests were made in the absence of an ionizing beam. The next significant step towards understanding the viability of a cooling channel using HPRF cavities is to study operation in an ionizing beam of appropriate intensity. The main focus of the MCTF RF activities in the last year has been to prepare a HPRF beam test in the MUCOOL Test Area (MTA). This requires extracting a linac beam and transporting it to the MTA. In the last year the beamline design has been completed, beamline components are have been refurbished and refitted, and four new elements built and installed: two identical beam stops and two extraction magnets (C-magnets). The beamline, instrumentation and vacuum elements, as well as utility systems, have also been installed. Completing the MTA beam capability and making the first HPRF test with beam is a priority for our MCTF activities in the coming year.

HCC Solenoid Development: The HCC requires the development of an appropriate magnet system. Two conceptually different designs have been investigated. The first is the large bore "conventional" design which consists of a solenoid coil with separate helical dipole and quadrupole coils wrapped around it. The second is a small bore "helical solenoid" consisting of many individual coils arranged in a helical pattern. The helical dipole and quadrupole components are generated by the coil offsets and radii. The helical solenoid version introduces some design constraints, but is significantly easier and cheaper to build, and has emerged as the preferred solution. As a first step toward building a helical solenoid magnet that can be tested in a beam, it is planned to build a subscale model with four solenoid coils that fits within the Technical Division Vertical Magnet Test Facility (VMTF) Dewar. The mechanical support structure for the test coils will also simulate the geometry of the solenoids in the real magnet. A magnetic measurement system will be developed to characterize the model magnet, validate the fields, and monitor field stability during current excitation.

Cooling Experiment: An important part of the future MCTF program is to perform an experimental test of the HCC theory, technology, and simulations. This will require developing and bench-testing $\mathrm{HCC}$ components, integrating them into a short test channel, and measuring the response of a muon beam to the channel. The test channel could be a prototype cooling section which includes all the components needed in real 
channel. An alternative strategy (MANX) is to build a simpler cheaper test channel designed specifically to demonstrate the cooling theory, but not using realistic cooling channel components that include RF. Which approach is optimal will depend upon costs, timescales and budgets. In the last year the MANX concept has been further developed and possible muon beams at Fermilab investigated. Further work must be done in the coming year to better understand the cost of implementing a muon beam, the cost of the MANX experiment, the cost of developing HCC components, and the cost of the alternative to MANX in which the developed HCC components are assembled into a short cooling section and tested in a beam.

HTS Conductor and Solenoid Studies: The final Muon Collider cooling channel stages may require DC solenoid magnets with magnetic fields of 40-50 $\mathrm{T}$ in an aperture of $\sim 50$ $\mathrm{mm}$. A $45 \mathrm{~T}$ hybrid solenoid with superconducting outer coils and copper inner coils has been successfully built and operated at the National High Magnetic Field Laboratory (NHMFL), however, the $\sim 30 \mathrm{MW}$ power consumption at the maximum field makes this magnet design unattractive, and perhaps impractical, for accelerator applications. A potentially more attractive solution is to use HTS conductor. To guide the design and simulation studies for the final cooling stages requires an initial HTS conductor and magnet R\&D activity to evaluate the practicality of developing high-field HTS solenoids, and assess the likely associated operating parameters. In the past 12 months studies have been performed on two kinds of HTS materials: BSCCO-2212 as a round multifilamentary wire, and anisotropic conductors, (BSCCO-2223 and second generation coated conductors YBCO). For the BSCCO-2212, tests have been performed to study the effects of $\mathrm{J}_{\mathrm{c}}$ from billet preparation and cabling parameters, primarily at $4.2 \mathrm{~K}$, along with SEM/EDS analysis. For the anisotropic conductors studies were performed on the angular dependence of the $\mathrm{J}_{\mathrm{c}}$ using a newly designed probe. All studies were performed in the Superconductor R\&D laboratory in the Fermilab Technical Division magnet systems department. In addition to conductor studies, HTS solenoid structural analysis and analytical optimization studies have been started to find an acceptable solution for the field, stresses and cost. Quench protection concepts are also being investigated. These studies will continue in the coming year, and should provide the basis for formulating a longer-term R\&D program and evaluating the likely properties of an HTS high-field solenoid build for a muon cooling channel.

National HTS Collaboration: The muon collider has multiple uses for very high field solenoids and dipoles. Fortunately there is an emerging technology that uses the High Temperature Superconductor, Bi2212 to achieve fields well beyond those available from niobium based superconductors. It has been discovered that operating Bi2212 at liquid He temperatures allows it to support very high current densities in ambient fields even beyond $50 \mathrm{~T}$. In addition, these materials seem to be very radiation resistant. Both of these properties are very useful in the design of a muon collider. As a result, the MCTF has taken the lead in the formation of a multi-laboratory consortium to develop this conductor and the technology for its use in dipoles and solenoids with fields greater than 20 T. Meetings have been held with LBL, LANL, BNL, NIST and NHMFL and agreement has been obtained to collaborate in exploring the development of these HTS conductors. An effective organization should be in place by the end of February 2008. However, for this group to function effectively, we must secure additional funding and that will be the central challenge over the next year. A letter has been sent to the DOE outlining a program that would cost about $\$ 2 \mathrm{M}$ per year spread over a five year period, an amount which is commensurate with the cost of developing NbSn-3 conductor. 
FY08 R\&D Activities: The main MCTF FY08 activities include (i) in collaboration with the NFMCC, compiling a complete list of R\&D questions to be addressed in the next 5 years, (ii) continued work on Muon Collider ring and cooling channel design and simulations, (iii) support of the continued NFMCC RF R\&D activities in the MTA, (iv) bringing beam to the MTA and supporting the first Muons Inc. HPRF cavity beam test, (v) construction of a four-coil HCC magnet prototype, (iv) continued HTS conductor studies and working towards a national HTS magnet collaboration, (v) continued studies of the options for a $6 \mathrm{D}$ cooling experiment and the development of HCC cooling channel components.

Summary: In its first year the MCTF has made progress on (i) Muon Collider ring studies, (ii) 6D cooling channel design and simulation studies with an emphasis on the HCC scheme, (iii) beam preparations for the first HPRF cavity beam test, (iv) preparations for an HCC four-coil test, (v) further development of the MANX experiment ideas and studies of the muon beam possibilities at Fermilab, (vi) studies of how to integrate RF into an HCC in preparation for a component development program, and (vii) HTS conductor and magnet studies to prepare for an evaluation of the prospects for of an HTS high-field solenoid build for a muon cooling channel. 


\section{Introduction}

Muon Colliders offer a possible long term path to lepton-lepton collisions at center-ofmass energies $V_{s} \geq 1 \mathrm{TeV}$. In October 2006 the Muon Collider Task Force (MCTF) proposed $\left[{ }^{1}\right]$ a program of advanced accelerator $R \& D$ aimed at developing the Muon Collider concept. The proposed R\&D program was motivated by progress on Muon Collider design in general, and in particular, by new ideas that have emerged on muon cooling channel design $\left[{ }^{2},{ }^{3}\right]$. The scope of the proposed MCTF R\&D program includes muon collider design studies, helical cooling channel design and simulation, high temperature superconducting solenoid studies, an experimental program using beams to test cooling channel rf cavities and a 6D cooling demonstration channel. The first year of MCTF activities was to make enough progress on these various sub-projects to be able to refine the R\&D plan once the level of support is established, and to conclude the first year's studies with a status report.

This MCTF status report summarizes results from the first year of MCTF activities, and describes an updated R\&D plan based on the anticipated level of support for FY08.

Table 2.1: Tentative parameters for different versions of the muon collider.

\begin{tabular}{|c|c|c|c|c|}
\hline & Low Emit. & High Emit. & MCTF06 & MCTF07 \\
\hline$V_{\mathrm{s}}(\mathrm{TeV})$ & \multicolumn{4}{|c|}{1.5} \\
\hline Av.Luminosity $\left(10^{34} / \mathrm{cm}^{2} / \mathrm{s}\right)^{*}$ & 2.7 & 1 & 1 & 1.33 \\
\hline Av. Bending field (T) & 10 & 6 & 8.33 & 6 \\
\hline Mean radius $(\mathrm{m})$ & 361.4 & 500 & 363.8 & 500 \\
\hline No. of IPs & 4 & 2 & 2 & 2 \\
\hline Proton Driver Rep (Hz) & 65 & 13 & 60 & 40 \\
\hline Beam-beam parameter/IP & 0.052 & 0.087 & 0.1 & 0.1 \\
\hline$\beta^{*}(\mathrm{~cm})$ & 0.5 & 1 & 3 & 1 \\
\hline Bunch length (cm) & 0.5 & 1 & 2 & 1 \\
\hline No. bunches / beam & 10 & 1 & 1 & 1 \\
\hline No. muons/bunch $\left(10^{11}\right)$ & 1 & 20 & 12 & 11.3 \\
\hline Norm. Trans. Emit. $(\mu \mathrm{m})$ & 2.1 & 25 & 13 & 12.3 \\
\hline Energy spread (\%) & 1 & 0.1 & 0.1 & 0.2 \\
\hline Norm. long. Emit. (m) & 0.35 & 0.07 & 0.14 & 0.14 \\
\hline $\begin{array}{l}\text { Total RF voltage }(\mathrm{GV}) \text { at } \\
800 \mathrm{MHz}\end{array}$ & $\begin{array}{l}406.6 \\
\times 10^{3} \alpha_{c}\end{array}$ & $0.21 * *$ & $0.26 \times 10^{3} \alpha_{c}$ & $0.84 * *$ \\
\hline Muon survival $\mathrm{N} \mu / \mathrm{N} \mu 0$ & 0.31 & 0.07 & 1 & 0.2 \\
\hline$\mu+$ in collision / proton & 0.047 & 0.01 & 0.15 & 0.03 \\
\hline $8 \mathrm{GeV}$ proton beam power & $3.62 * * *$ & 3.2 & $0.6 f_{\text {loss }}^{*}$ & 1.9 \\
\hline
\end{tabular}

*) Luminosity calculated taking account of the hour-glass factor but ignoring the dynamic beta effect.

**) Momentum compaction in the present ring design $\alpha_{c}=1.5 \times 10^{-4}$. Note that it would be better to assume $\mathrm{f}=1.3 \mathrm{GHz}$ to keep the $\mathrm{RF}$ voltage at a reasonable level.

***) Assumes $\mu / \mathrm{p}$ ratio of 0.15 after capture and precooling, and only decay losses afterwards. Positive and negative muons are assumed to be produced independently (from different protons).

*) $\mathrm{f}_{\text {loss }}=$ muon losses during cooling and acceleration, which has not yet been fully simulated. 


\section{Muon Collider Design Studies}

A Muon Collider requires an intense source of cold muons. The muons can be produced using a high intensity proton source, with a primary beam energy of a few $\mathrm{GeV}$ or higher. The proton beam interacts in the target to produce low energy charged pions, which are collected in a high-field solenoid channel, and decay to produce an intense but diffuse beam of muons. The muons must be captured longitudinally and, to obtain a reasonable collider luminosity, before the muons decay the $6 \mathrm{D}$ phase space occupied by the initial muon population must be reduced by a factor $\mathrm{O}\left(10^{6}\right)$. The exact requirements for the proton beam power and the cooling channel performance depend on the collider parameters.

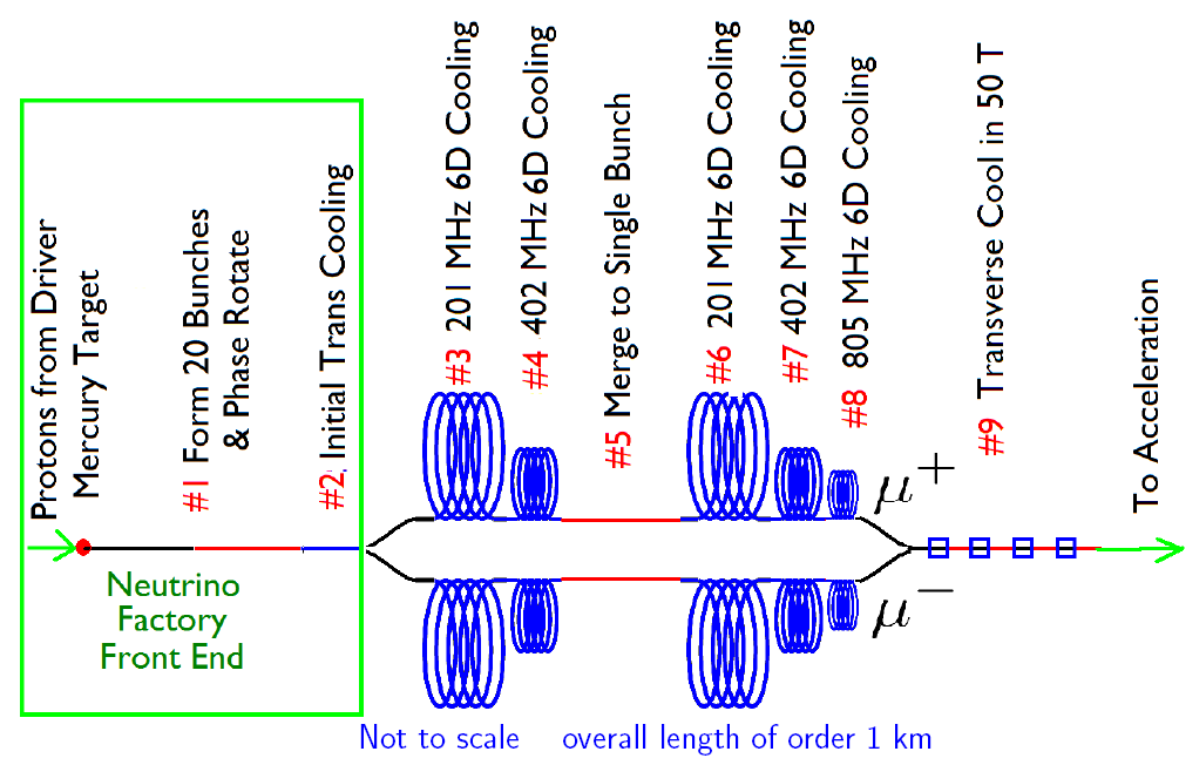

Figure 2.1: Front-end for the high-emittance Muon Collider Scheme.

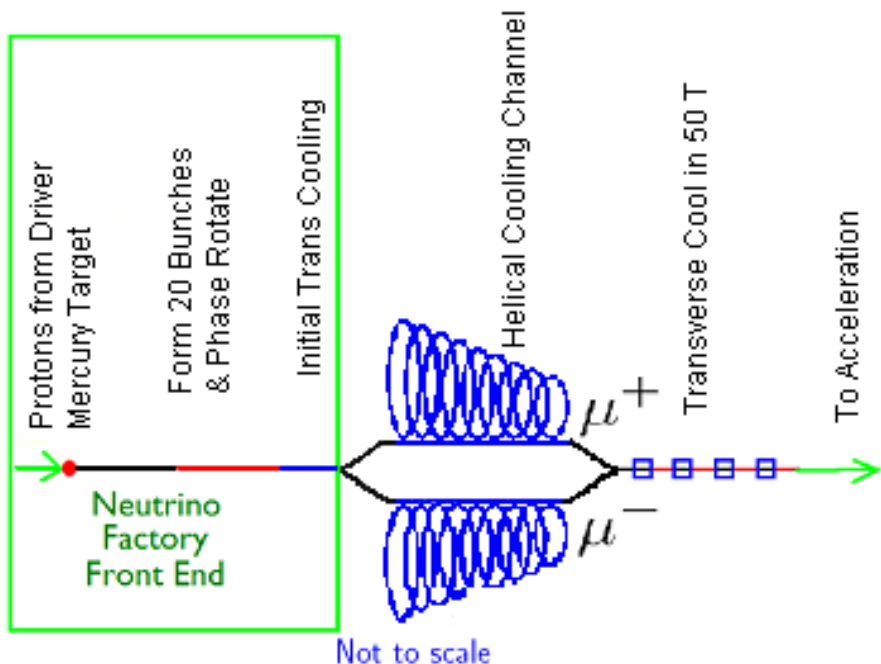

Figure 2.2: Front-end for a high-emittance Muon Collider scheme using a helical cooling channel. 
Different Muon Collider schemes, corresponding to different collider parameter sets, have been proposed. These schemes use a variety of different cooling channel technologies for reducing the 6D phase space. Table 2.1 summarizes the parameter sets that correspond to representative muon collider schemes. The table shows the parameter sets as they were when the MCTF was initiated, and how they have evolved over the last year. Two representative Muon Collider front-ends are shown schematically in Figs. 2.1 and 2.2.

\subsection{Muon Collider Parameter Choices}

An important goal for the MCTF activity is, within the next two to three years, to establish one or more realizable muon collider parameter set(s), and hence identify the performance goals for the muon cooling channel.

Past physics studies $\left[{ }^{4}\right]$ have shown that a Muon Collider with $\sqrt{s}_{\mathrm{s}}=1 \mathrm{TeV}$ to a few TeV requires a minimum luminosity of $\sim 10^{34} \mathrm{~cm}^{-2} \mathrm{~s}^{-1}$ to probe with adequate sensitivity the physics beyond the Standard Model. The peak luminosity in colliding beams of transverse emittance $\varepsilon_{\perp}$ and intensity $n_{b} N_{\mu}$ is given by

$$
\mathcal{L} \sim \frac{n_{b} N_{\mu}^{2}}{\varepsilon_{\perp} \beta^{*}} h\left(\sigma_{z} / \beta^{*}\right) \sim \frac{n_{b} N_{\mu} \xi}{\beta^{*}} h\left(\sigma_{z} / \beta^{*}\right)
$$

where $h$ is the so-called hour-glass factor, and

$$
\xi=\frac{r_{\mu} N_{\mu}}{4 \pi \varepsilon_{\perp N}}
$$

is the beam-beam parameter. Once the beam-beam limit is reached $\left(N_{I P} \xi<0.2\right)$, lowering the transverse emittance no longer directly increases the luminosity. To benefit from further emittance reduction requires lowering the number of muons per bunch $N_{\mu}$, and increasing the number of bunches $n_{b}$. With these considerations in mind, two design strategies have been proposed to achieve a high luminosity Muon Collider. In strategy 1 , the muons of each sign are packaged in the minimum number of bunches (one per cycle) and the emittance goal corresponds to the beam-beam limit. This strategy leads to a so called "high emittance" design. The latest set of parameters for the high emittance design is shown in the second column of parameters in Table 2.1. The repetition rate is chosen to be relatively low $(13 \mathrm{~Hz})$ to keep the proton driver beam power reasonable. In strategy 2 , the muons are packaged into many bunches with much lower transverse emittances. An initial set of parameters for the low emittance design is shown in the first column of parameters in Table 2.1.

The low emittance option allows higher beta-function values in the final focus triplets and correspondingly lower beta values at the IPs (as small as a few $\mathrm{mm}$ ). To benefit from this, the bunch length must be small: $\sigma_{\mathrm{s}}<\beta^{*}$. Note that the RF voltage needed for longitudinal focusing increases with decreasing bunch length:

$$
V_{R F} \sim \alpha_{c} \sigma_{p}^{2} / \sigma_{s}^{2}=\alpha_{c} \varepsilon_{\|}^{2} / \sigma_{s}^{4}
$$


where $\sigma_{p}=\varepsilon_{\|} / \sigma_{\mathrm{s}}$ is the momentum spread and $\varepsilon_{\|}$is the longitudinal emittance. To keep $\mathrm{V}_{\mathrm{RF}}$ within reasonable limits requires a very low momentum compaction factor $\alpha_{c}$. A low $\alpha_{c}$ lattice creates strong chromatic perturbations which must be corrected over a wide momentum range. In addition, given the large number of bunches, it will be necessary to either separate the beams in the parasitic IPs with the help of electrostatic separators, or resort to a two-ring scheme. On the other hand, the smaller number of particles per bunch would alleviate problems associated with the space charge and coherent instabilities all the way from the proton driver to the collider, and smaller transverse emittances would permit the use of lower acceptance accelerators.

\subsection{Muon Collider Ring Studies}

To establish the feasibility of the high-and/or low-emittance schemes it is necessary to establish the existence of corresponding Collider Lattice designs that assume realistic parameters for magnets, rf, etc.

In order to obtain a luminosity in the $10^{34} \mathrm{~cm}^{-2} \mathrm{~s}^{-1}$ range, the beta function at the IP should be $\sim 1 \mathrm{~cm}$ or less. Given realistic quadrupole gradients, the low beta quadrupoles must be placed relatively far from the IP, resulting in a large beta function at the quadrupoles, and high chromaticity. A typical value of the Montague chromatic functions is $4 \times 10^{3}$. The only known way to deal with such strong perturbations is to compensate them locally with sextupoles, before the betatron phase advance appreciably changes (otherwise there will be a $400 \%$ beta function modulation for a momentum deviation $\delta_{p}=0.001$ ). In addition, the muon collider requires a low value of $\alpha_{c}$. The arc lattice proposed in Ref. $\left[{ }^{5}\right]$ has a small and negative $\alpha_{c} \approx-10^{-5}$, obtained at the price of introducing a very strong chromaticity, comparable to that of the interaction region. The resulting momentum acceptance is very small $(<0.07 \%)$. To obtain a larger momentum acceptance, a new design has been investigated which has the following features:

- local chromatic compensation with sextupoles in the IR and dispersion generated by dipoles placed near to the IPs ;

- a low momentum compaction factor obtained by balancing the positive contribution from the arcs with the negative contribution from the system that suppresses the dispersion generated in the IR ;

- high phase advance $(3 \pi / 5)$ FODO arc cells to maximize the dipole packing factor and hence minimize the collider circumference;

- octupoles to correct the nonlinear amplitude dependent detuning produced by the IR sextupoles. 

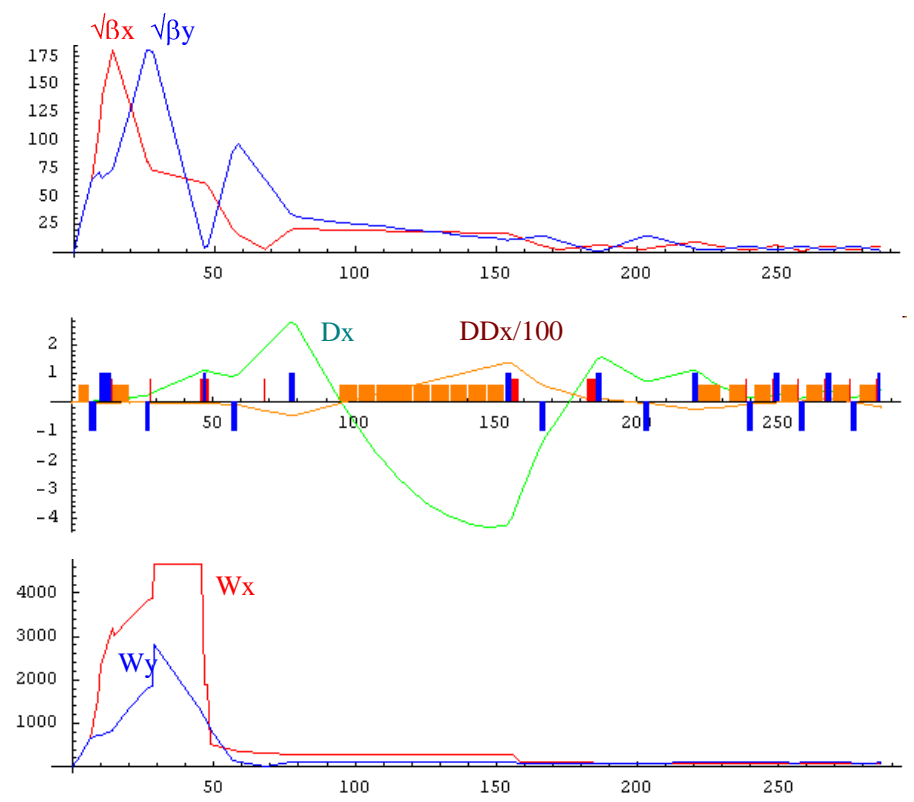

Figure 2.3: Square root of beta functions (top), first and second order dispersion functions (middle) and chromatic functions (bottom) vs distance from IP. The middle plot shows lattice elements: orange - dipoles, blue - quadrupoles, red - sextupoles (polarity not indicated). Octupoles are not shown.
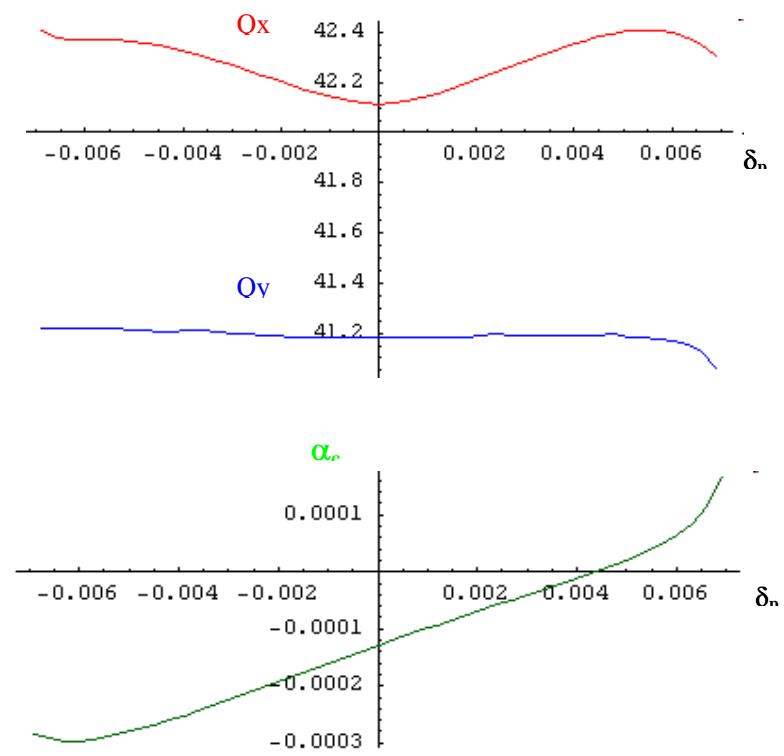

Figure 2.4: Betatron tunes (top) and the momentum compaction factor (bottom) vs relative momentum deviation.

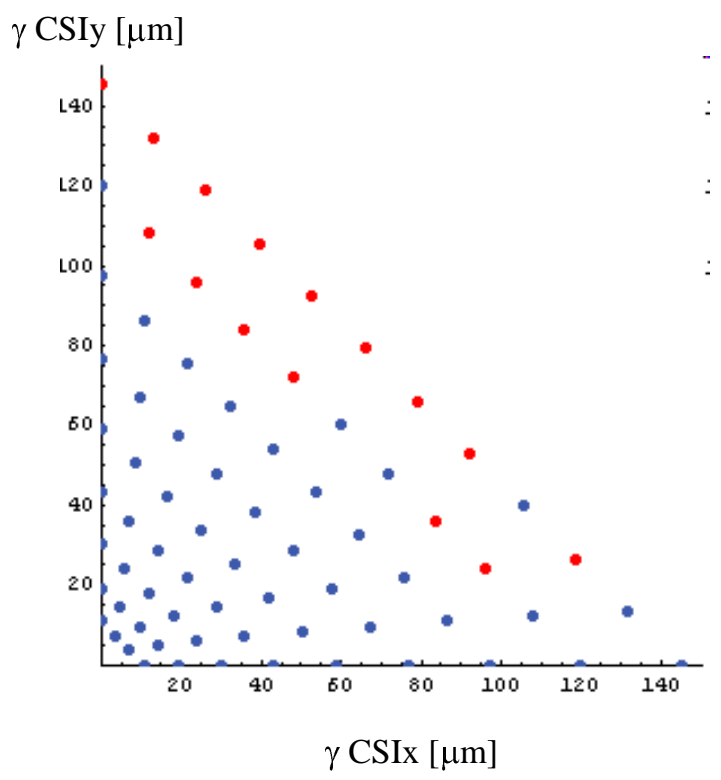

Figure 2.5: 1024 turns dynamic aperture in the plane of normalized CourantSnyder invariants: blue - survived particles, red-lost particles. 


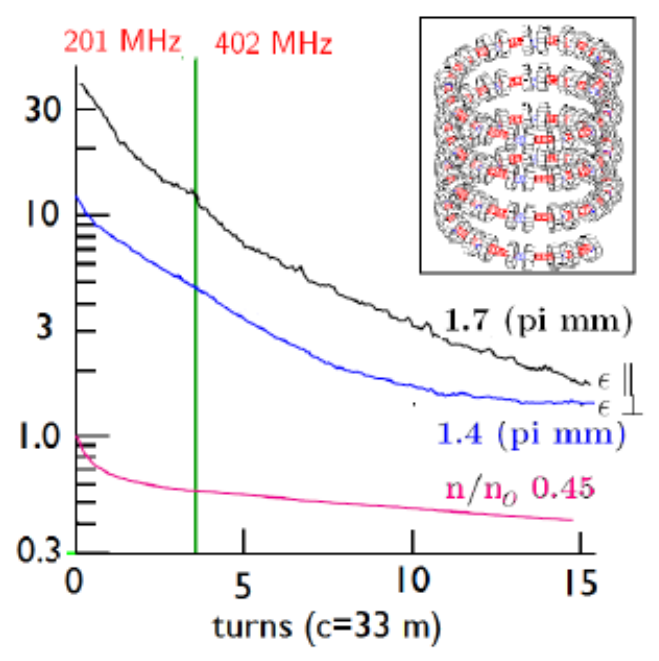

Figure 2.6: ICOOL simulation of $6 D$ cooling in stages \#3 \& \#4. Inset: Geometry of \#3

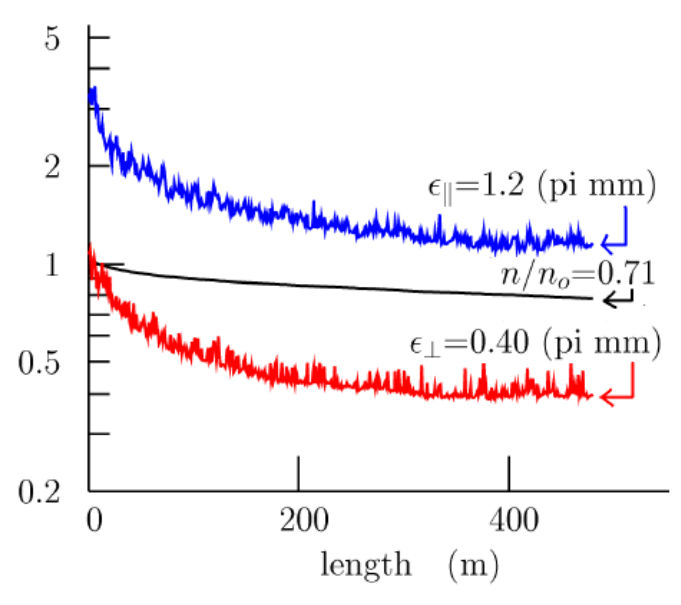

Figure 2.7: ICOOL simulation of final $6 D$ cooling lattice (\#8) using 10T solenoids and $805 \mathrm{MHz} r f$.

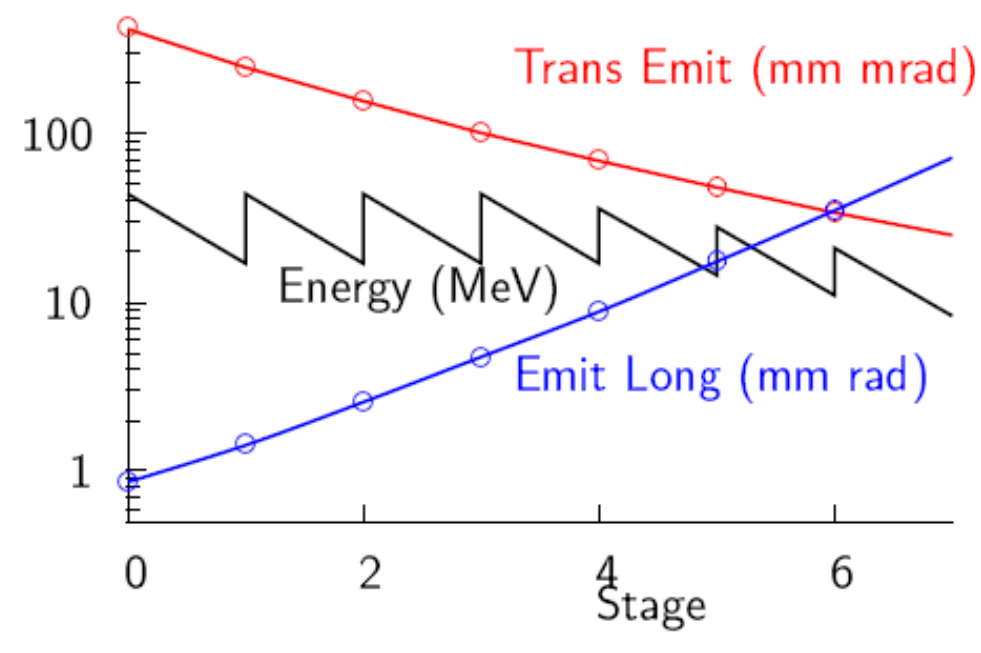

Figure 2.8: Results of ICOOL simulations of transverse cooling in liquid hydrogen in 7 sequential 50 T solenoids. 
Figure 2.2 shows optics functions in the IR, negative dispersion suppressor and matching section for $\beta^{*}=1 \mathrm{~cm}$. The stability properties of the lattice are illustrated by Figs. 2.3 and 2.4. The momentum acceptance is $\pm 0.7 \%$ (Fig. 2.3), however the momentum compaction factor changes its sign within this range so that further adjustments are necessary. Figure 4 shows the short-term dynamic aperture which is about $3 \sigma$ for the normalized emittance $12.5 \mu \mathrm{m}$. The momentum acceptance is too small for the low emittance design, although the dynamic aperture is more than sufficient. It seems unlikely that the lattice design can be modified to meet the low emittance collider requirements. For the high emittance option the dynamic aperture is half of what is needed, and the momentum acceptance is twice as large as needed. Probably the dynamic aperture can be increased by lowering the sextupole strength and thus sacrificing some momentum acceptance. Another possibility is to reduce the number of IPs to 1 .

In summary, there has been progress in understanding the Muon Collider ring design issues for the low- and high-emittance schemes. Present designs for the high-emittance lattice are close to achieving the performance that corresponds to the high-emittance parameter set in Table 2.1. However, new ideas are needed to achieve a ring design that corresponds to the low emittance parameters. Finally, it should be noted that past studies have shown that very detailed studies are required to understand how to adequately shield the Muon Collider detector from the copious backgrounds associated with the daughter electrons from muons decaying as they approach the IP. For any new final focus design, these studies must be made before we can have confidence that the design is practical.

\subsection{Muon Collider Front End}

The front-end of the Muon Collider, which we define as everything up to, but not including, the acceleration stages, consists of a proton source, target station, pion collection and decay channel, muon bunching system, phase rotation system (to reduce the energy spread of the captured muons, and finally a muon ionization cooling system to reduce the $6 \mathrm{D}$ phase space occupied by the muons by $\mathrm{O}\left(10^{6}\right)$.

\subsubsection{High Emittance Collider Scenario}

A representative high emittance scheme is being pursued by members of the MCTF in collaboration with the NFMCC. The present design corresponds to the high emittance parameter set listed in Table 2.1. This scheme would yield a $1.5 \mathrm{TeV}$ Muon Collider with a luminosity of $1 \times 10^{34} \mathrm{~cm}^{-2} \mathrm{~s}^{-1}$.

The high emittance scheme is shown in Fig. 2.1. Muons are generated by the decay of pions produced by proton bunches interacting in a mercury jet target. The pions are captured by a $20 \mathrm{~T}$ solenoid surrounding the target, followed by a larger radius lower field decay channel. The first manipulation (\#1), referred to as phase rotation, converts the initial single short muon bunch into a train of 21 bunches with much reduced energy spread. The initial bunch is allowed to lengthen and develop a time-energy correlation in a $110 \mathrm{~m}$ drift. It is then bunched into a train, without changing the time-energy correlation, using RF cavities whose frequency varies with location, falling from 333 $\mathrm{MHz}$ to $201 \mathrm{MHz}$. Using phase and frequency control, the RF accelerates the low energy bunches and decelerates the high energy ones. Muons of both signs are captured and then (\#2) cooled transversely in a linear channel using LiH absorbers, alternating periodically 
with $2.8 \mathrm{~T}$ solenoids and $201 \mathrm{MHz}$ RF cavities. All the components up to this point are identical to those described in a recent study $\left[{ }^{6}\right]$ for a neutrino factory. The next stage (\#3) cools simultaneously in all 6 dimensions. The lattice $\left[{ }^{7}\right]$, which has been called a "Guggenheim lattice", uses 3 T solenoids for focus, weak dipoles (generated by tilting the solenoids) to generate dispersion, wedge shaped liquid hydrogen filled absorbers where the cooling takes place, and $201 \mathrm{MHz}$ RF, to replenish the energy lost in the absorbers. The dipole fields cause the lattices to curve, forming a slow upward or downward helix (see inset in Fig. 2.6). The following stage (\#4) uses a Guggenheim lattice essentially the same as \#3, but with twice the field strength, half the geometric dimensions, and $402 \mathrm{MHz}$ RF. Figure 2.6 shows the results of a simulation of both systems using ICOOL. The next step (\#5) is to combine the bunches into one bunch of each sign, which is done in two stages: a) using a drift followed by $201 \mathrm{MHz}$ RF, with harmonics, the individual bunches are phase rotated to fill the spaces between bunches and lower their energy spread; followed by b) $5 \mathrm{MHz} \mathrm{RF}$, plus harmonics, interspersed along a long drift to phase rotate the train into a single bunch that can be captured using $201 \mathrm{MHz}$ RF. After the bunch merging, the longitudinal emittance of the single bunch is now similar to that at the start of step \#3. It can thus be taken through the same, or similar, cooling systems as \#3 and \#4: now numbered \#6 and \#7. One more Guggenheim stage (\#8) of 6D cooling follows \#6 and \#7, using 10T magnets, hydrogen wedge absorbers, and $805 \mathrm{MHz}$ RF. The ICOOL simulated performance, using fields that, while they satisfied Maxwell's equations and had realistic strengths, were not actually calculated from specified coils, is show in Fig. 2.7. To attain the required final transverse emittance, the cooling needs stronger focusing than is achievable in the Guggenheim cooling lattices. It is obtained in stage \#9 using liquid hydrogen in strong (50T) solenoids, allowing the momentum to fall and the momentum spread, and thus longitudinal emittance, to rise. Figure 2.8 shows the results of ICOOL simulation of cooling in seven $50 \mathrm{~T}$ solenoids. The simulation did not include the required matching and re-accelerations between the solenoids.

\subsubsection{Low Emittance Collider Scenario}

A representative low emittance scheme is being pursued by members of the MCTF in collaboration with Muons Inc. The present design concept corresponds to the low emittance parameter set listed in Table 1. This scheme would yield a 1.5 TeV Muon Collider with an average luminosity of $2.7 \times 10^{34} \mathrm{~cm}^{-2} \mathrm{~s}^{-1}$

In contrast to the high emittance scheme, in the low emittance design:

1) the collider ring contains multiple muon bunches, which require a proton driver beam with a multi-bunch structure for the muon production;

2) the $\mu^{+}$and $\mu^{-}$are separately produced to simplify their RF capture and bunch rotation, with each muon beam consisting of $n_{b}$ batches of $\sim 10$ low-intensity muon bunches;

3) the muon cooling channel must yield lower emittances, which may be accomplished using so-called parametric ionization cooling (PIC) in addition to reverse emittance exchange (REMEX) in a 50T solenoid channel; 
4) the bunches are not merged in the cooling channel, but batches of low-intensity muon bunches are coalesced into $2 \times n_{b}$ bunches at higher energy $(20-30 \mathrm{GeV})$.

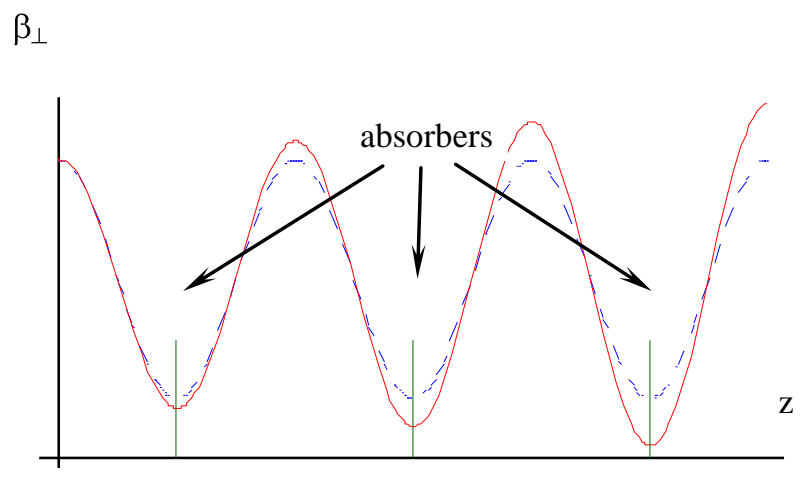

Figure 2.9: Schematic of the PIC principle.
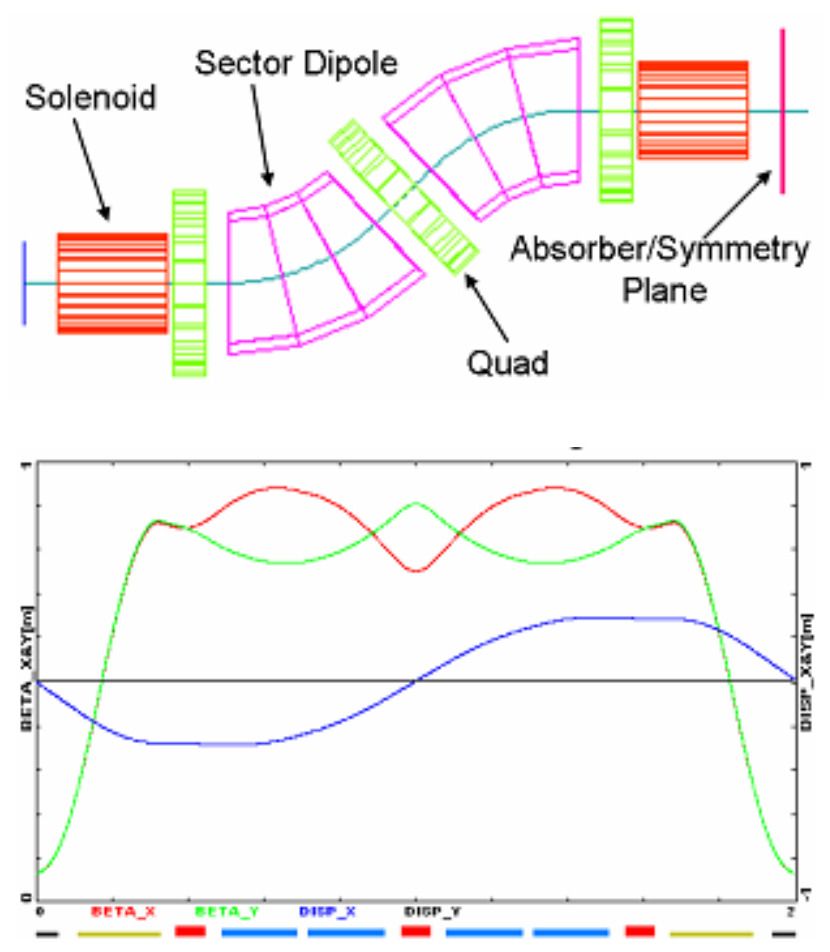

Figure 2.10: PIC cell layout and optics functions.

Note that, since the proton and muon beams are packaged into a large number of bunches, potential problems with space charge effects and single bunch instabilities are expected to be mitigated. However, the present scheme depends on the feasibility of PIC and REMEX. 
The principle of PIC is illustrated by Fig. 2.9: the blue dashed line shows the beta function of the unperturbed lattice. The tune is set on the boundary of the half-integer (parametric) resonance. The absorbers (green vertical lines) are placed at the minima of the beta function. Adding small focusing perturbations excites a growing beta-wave (red line). With appropriate choice of the phase of the perturbation, the beta function at the absorbers, and therefore the equilibrium emittance, will gradually decrease down the channel. Owing to the emittance reduction, the beam size at the maxima should not increase despite the growing beta-wave. It is hoped that beta function values in the submillimeter range can be achieved. However, compensation of chromatic and spherical aberrations is expected to be challenging. Figure 2.10 (top) shows a PIC cell layout $\left[{ }^{8}\right]$. It contains solenoids, quadrupoles, and bends which produce the dispersion necessary for chromatic correction and longitudinal cooling via emittance exchange using wedge shaped absorbers. The optics functions in the detuned cell are shown in Fig. 2.10 (bottom). Compensation of aberrations with the addition of sextupole and higher multipole field components has yet to be demonstrated. A viable collider ring scheme that provides sufficient momentum acceptance will also be required (see section 2.2).

\subsubsection{Cooling Schemes using a Helical Cooling Channel}

In addition to different collider design strategies (low- or high-emittance), different cooling channel technologies are also being explored. In particular, the idea of using a helical solenoid to mix the longitudinal- and transverse-degrees of freedom has been proposed by Derbenev $\left[{ }^{9}\right]$, and developed by the NFMCC $\left[{ }^{10}\right]$, and Muons Inc. $\left[{ }^{11}\right]$. The most advanced helical solenoid cooling (HCC) scheme, developed by Muons Inc., consists of a helical magnet filled with a high pressure gas (e.g. hydrogen) which provides a continuous ionization cooling absorber. Initial studies indicate that this scheme can achieve the same cooling factor as the Guggenheim channel, but in a shorter length, which will hopefully improve the transmission efficiency. Figure 2.11 shows the results from a G4beamline simulation of the longitudinal-transverse emittance evolution in the cooling channel. The transverse and longitudinal emittances at the input to the $160 \mathrm{~m}$ long channel are both $200 \mathrm{~mm}$. The transverse emittance at the end is $<1 \mathrm{~mm}$, while the longitudinal emittance has been reduced is to $\sim 1 \mathrm{~mm}$. These emittances are obtained in the current $\mathrm{HCC}$ design with the maximum field strength of $\sim 17$ Tesla.

At the end of the $160 \mathrm{~m}$ long HCC channel the longitudinal emittance already satisfies the goal for the high emittance collider scenario, but the transverse emittance is a factor of 2.5 larger than required. To attain the transverse emittance goal, a high field HCC, with a maximum field more than 20 Tesla, is being studied. The HCC channel may therefore provide an alternative to the Guggenhiem channel for a high emittance collider scheme, or provide the initial cooling (prior to PIC, see Fig. 2.11) in a low emittance scheme. At the end of the PIC stage, the longitudinal emittance is much smaller than required, and can therefore be allowed to grow in exchange for further transverse cooling. Therefore, PIC could be followed by a high-field solenoid channel (see section 2.3.1), or some alternative "reverse emittance exchange" (REMEX $\left[{ }^{12}\right]$ ), to attain final emittances of 2 $\mu \mathrm{m}$ transverse and $20 \mathrm{~mm}$ longitudinal. 


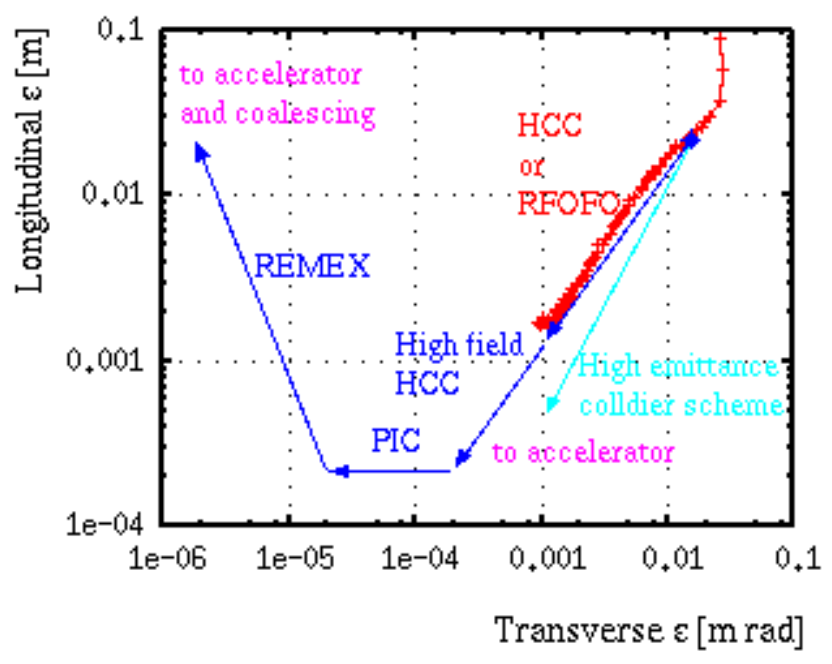

Figure 2.11: Longitudinal-Transverse emittance evolution plot for schemes with an initial HCC. The red points show simulation results. The blue lines show the desired path in the present conceptual scheme.

\subsubsection{Muon Ring Coolers}

In addition to linear cooling channels, muon ring coolers are also being studied by UCLA-BNL collaborators $\left[{ }^{13}\right]$ as potentially more cost effective cooling systems. While there are many versions of ring coolers, those that use dipoles and solenoids in combination seem very promising. The edge focusing of the dipole, combined with 4 $10 \mathrm{~T}$ solenoids to give very low beta, can lead to strong 6D cooling. Figure 2.12 shows a schematic of a pure dipole cooler and Fig. 2.13 shows a Li lens ring cooler. Another scheme is to use a bent $\mathrm{Li}$ lens system $\left[{ }^{14}\right]$. The challenges for these interesting ring cooler schemes include designing an effective injection and extraction scheme, and developing components with the required performance.

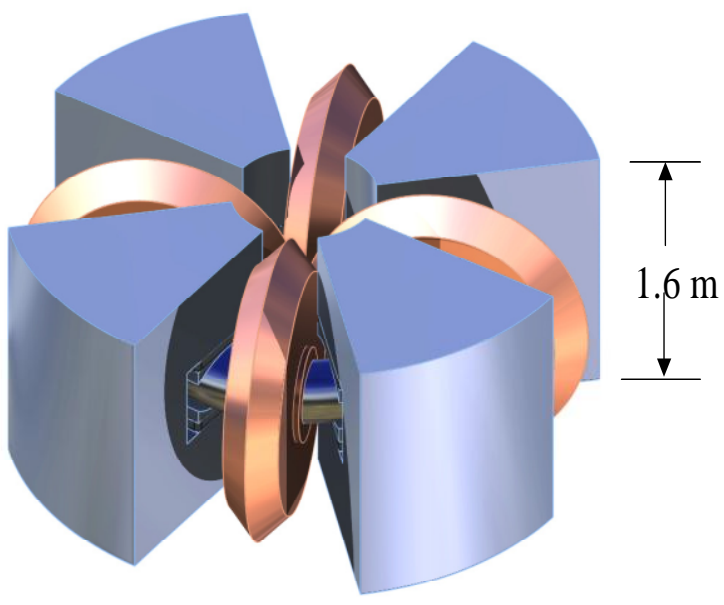

Figure 2.12: Ring cooler with dipole. 


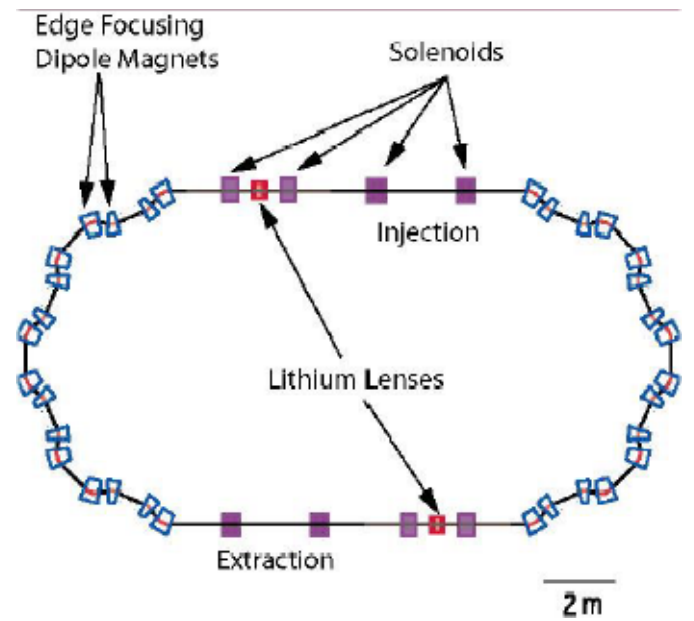

Figure 2.13: A schematic diagram of a muon cooling ring with straight Lithium lenses.

\subsubsection{Summary of Cooling Channel Options}

An important ongoing MCTF activity is to sort through the various cooling channel technologies and design strategies, and make more complete studies of the designs that appear most promising. Ongoing studies are looking at designs in which there is one muon bunch or many muon bunches per cycle. Once these extreme cases are understood, intermediate cases must be explored to find the optimum number of muon bunches per cycle. In addition, a significant number of potential cooling channel technologies are being explored, including Guggenheim, HTS, HCC, PIC, REMEX, rebunching schemes, schemes with high pressure RF, pillbox vacuum RF, magnetically insulated open cell RF, etc. In this exploratory stage in Muon Collider cooling channel design, to inform the choices that must eventually be made, in the coming year we anticipate continuing to develop the various concepts and extending the associated simulation studies. 


\section{High Pressure RF Studies}

The present designs for the phase rotation and cooling channels require high gradient $\mathrm{RF}$ cavities to operate in magnetic fields of up to a few Tesla. This requirement excludes using superconducting RF (SCRF). Pillbox cavities, in which the normally open iris is closed with a thin conducting window, are preferred since, compared to open cell geometries, for a fixed peak RF power, the accelerating field on axis is about a factor of two higher. Channels using pillbox cavities therefore require about a factor of two fewer RF power sources. However, the NFMCC MUCOOL R\&D has revealed that high magnetic fields degrade the performance of normal conducting RF (NCRF) copper pillbox cavities with thin Be windows. In the next one to two years, the MUCOOL program will investigate the effects of using different materials and surface preparations to see if the limitations can be mitigated. Earlier MUCOOL studies have suggested that the performance of open cell cavities do not degrade significantly in high magnetic fields, although this needs to be confirmed. Unfortunately, due to the required larger number of RF power sources, an open cell solution will be more costly. A third potentially cost effective solution has been proposed for cooling channel RF cavities in which high pressure hydrogen gas fills the cavity (HPRF). The high pressure gas acts as the $\mathrm{dE} / \mathrm{dx}$ absorber and also suppresses the dark current in the cavity, and hence mitigates breakdown effects. In addition, the flowing dense gas can be used to cool down the cavity wall and the entrance window, which are heated by the energy deposition from the a beam and the resistive RF heating. Initial tests of HPRF cavities have shown that the required gradients can be obtained even when operating in magnetic fields up to 3 Tesla. However, operation of HPRF cavities has not been demonstrated in the presence of an ionizing beam. Hence, to understand whether this new muon cooling channel RF concept is viable, a HPRF cavity test must be made in an ionizing beam.

\subsection{Initial HPRF Cavity Tests}

In previous years, a HPRF test cavity has been built using Muons Inc. SBIR funding and, in collaboration with Fermilab, filled with high pressure gas and tested $\left[{ }^{15},{ }^{16},{ }^{17},{ }^{19},{ }^{19}\right]$ in a 5T solenoid using the MUCOOL facilities. These initial tests yielded promising results.

Figure 3.1 shows a schematic of the Muons Inc. HPRF test cell (TC), and Fig. 3.2 shows the TC located in the MUCOOL solenoid. The TC is made from a stainless steel cylinder with top and bottom plates designed to contain hydrogen gas at pressures up to 1600 PSIA. A thin aluminum plate is used as a sealing gasket. The surface of the TC is copper plated to reduce resistive RF heating. There are two hemispherical electrodes in the TC. The design localizes the maximum electric field at the top of the electrodes, and enables the electrodes to be interchanged to facilitate investigating the breakdown characteristics of different materials. $\mathrm{Cu}, \mathrm{Be}, \mathrm{Mo}$, and $\mathrm{W}$ electrodes have been tested. There are three ports connecting to the TC which permit a RF power probe to excite the RF resonance field, a pickup probe to measure the excited RF voltage, and a gas inlet (not indicate in Fig. 3.1). The RF power is generated by a Fermilab linac test station klystron, and transmitted to the MUCOOL Test Area (MTA) via a 90-m waveguide and coaxial cable. The klystron can operate within the frequency range $805 \pm 5 \mathrm{MHz}$. The gas pressure is monitored using a pressure indicator with a reading error less than 5 PSIA. Note that the capacitance of the TC varies with pressure (and hence gas density) causing a shift in the resonance frequency. Figure 3.3 shows the observed frequency shift, measured with a network analyzer, as a function of gas density. The resonant frequency changes linearly with gas density, and the measured frequency can therefore be used to determine the gas 
density in the TC. The resonant frequency drifted typically within 0.2 to $0.3 \mathrm{MHz}$ during data taking.

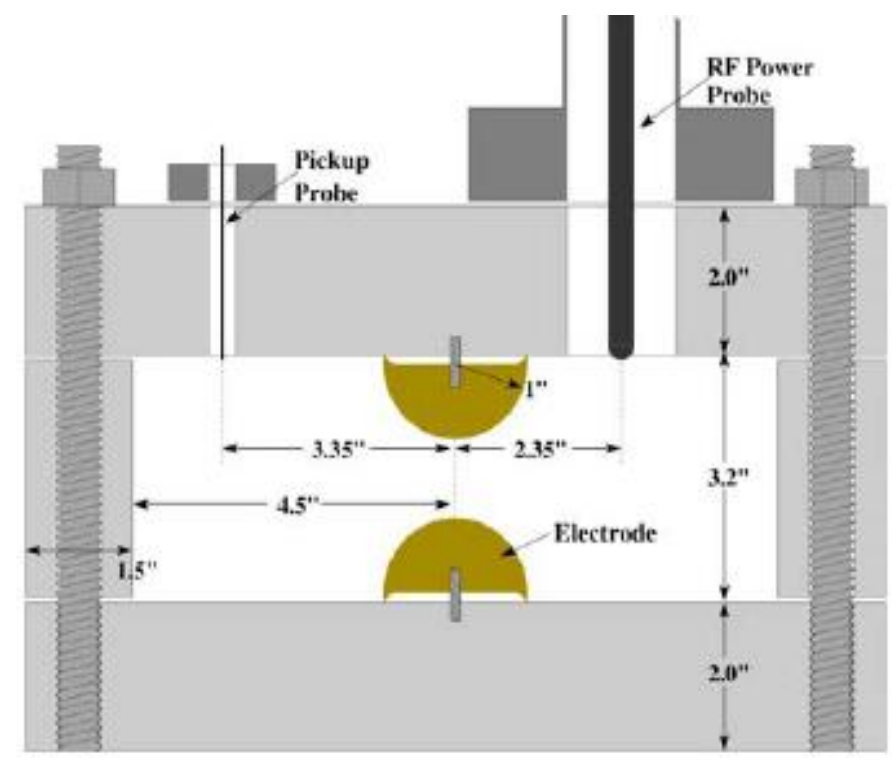

Figure 3.1: Cross-section of the Muons, Inc. HPRF test cell.

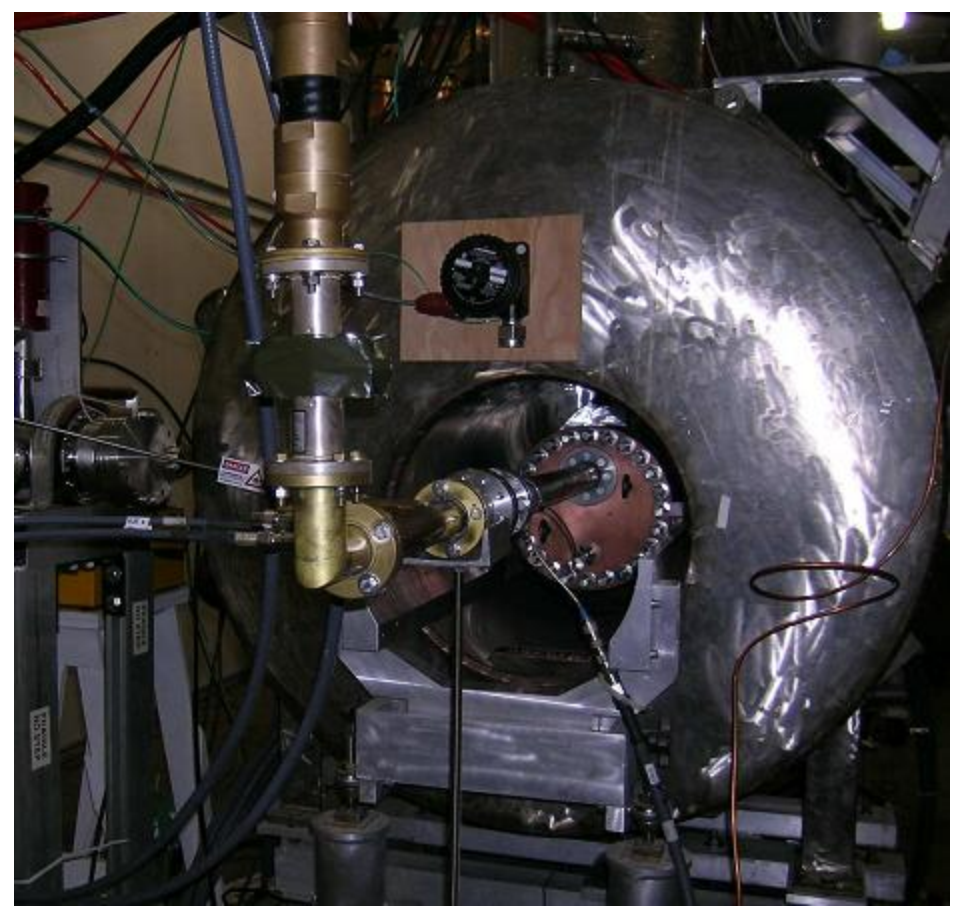

Figure 3.2: The TC (a brown cylinder) is mounted on the support rail in the solenoid magnet (a big silver cylinder). The TC is connected to the power probe (a thick coaxial cable) and the pickup probe (a thin coaxial cable). The gas inlet line is hidden behind the power probe. 
Figure 3.4 shows, for various electrode materials, as a function of the gas density, the observed amplitude of the RF breakdown field. The observed breakdown field shows a linear dependence on gas density and reaches a maximum at $0.005 \mathrm{~g} / \mathrm{cm}^{3}$ independent of the electrode material. This is the region of gas breakdown. The slope in gaseous helium is about six times smaller than in gaseous hydrogen. RF breakdown measurements were also made in a 3T field using Mo electrodes. No degradation of the RF breakdown field was observed. The breakdown field reaches a plateau at a hydrogen gas density above $0.005 \mathrm{~g} / \mathrm{cm}^{3}$. This is the region of the metallic breakdown where the maximum RF field is determined by the characteristics of electrode material.

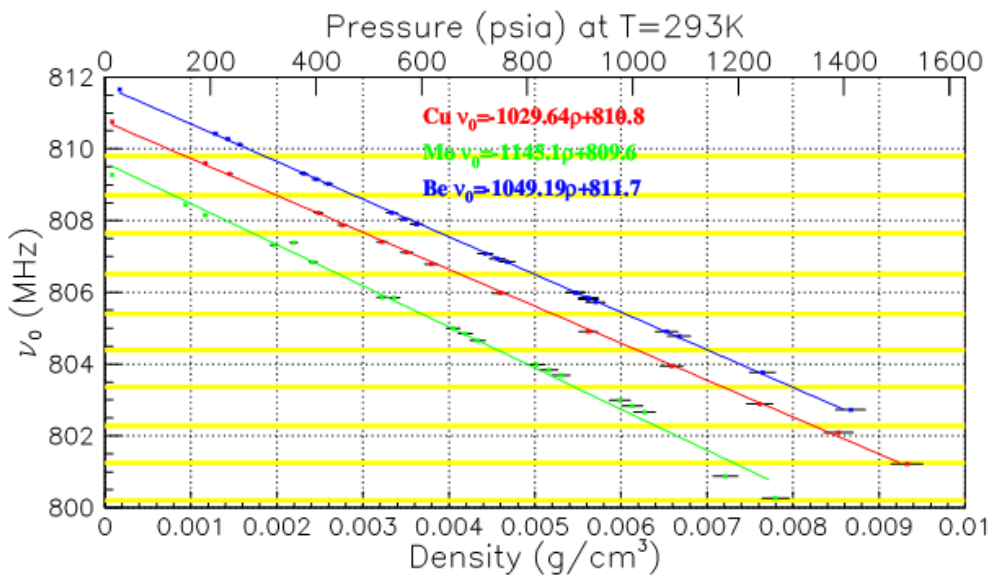

Figure 3.3: Observed resonant frequency in the TC as a function of the gas density (pressure) with various electrode materials by using the network analyzer. Yellow bands show the resonance in the waveguide.

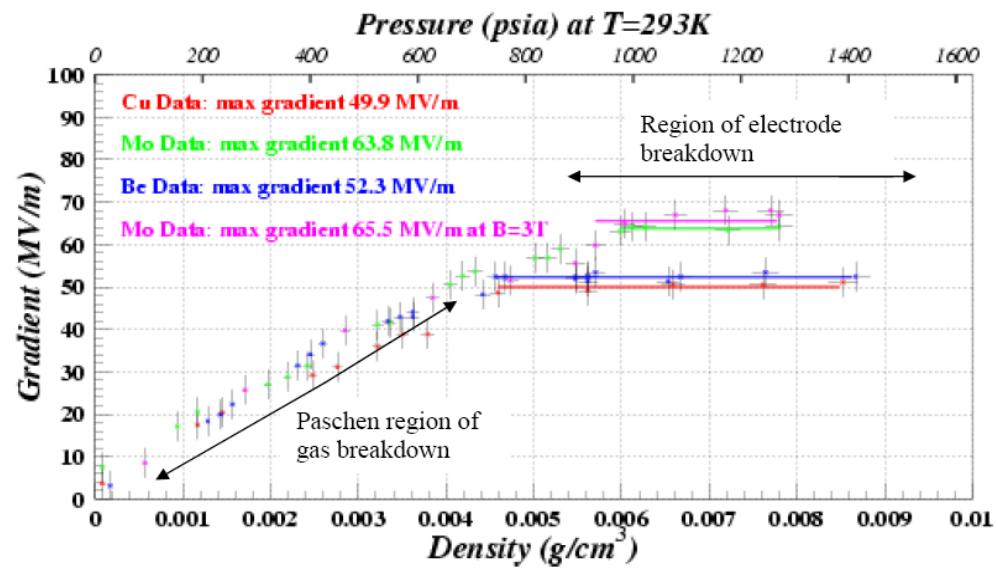

Figure 3.4: Observed RF breakdown field gradient in the TC as a function of the gas density (pressure) with various electrode materials. 


\subsection{HPRF Cavity Beam Tests}

The initial HPRF cavity tests yielded promising results, but this was without the presence of an ionizing beam. It is possible that the secondary electrons generated by the beam will live long enough in the cavity to cause problems $\left[{ }^{20}\right]$. To demonstrate the feasibility of using a HPRF cavity filled with hydrogen in a muon cooling channel it is therefore necessary to demonstrate operation in the presence of an ionizing beam. In the coming year we plan to test a HPRF cavity filled with hydrogen in the MUCOOL Test Area (MTA) located at the end of the Fermilab $400 \mathrm{MeV}$ linac. The MTA was built several years ago using NFMCC funding, together with Fermilab effort. This initial funding was insufficient to build a beamline to bring beam from the linac to the MTA, but provision was made to implement this at a later date. More recently some NFMCC funds were made available to help initiate implementing the beam, and in FY07 the first MCTF M\&S has been applied to prepare this beamline for a HPRF test in FY08.

\subsubsection{MTA Beamline}

The MTA beam transfer line layout is shown in Fig. 3.5, and the associated parameters are listed in Table 3.1

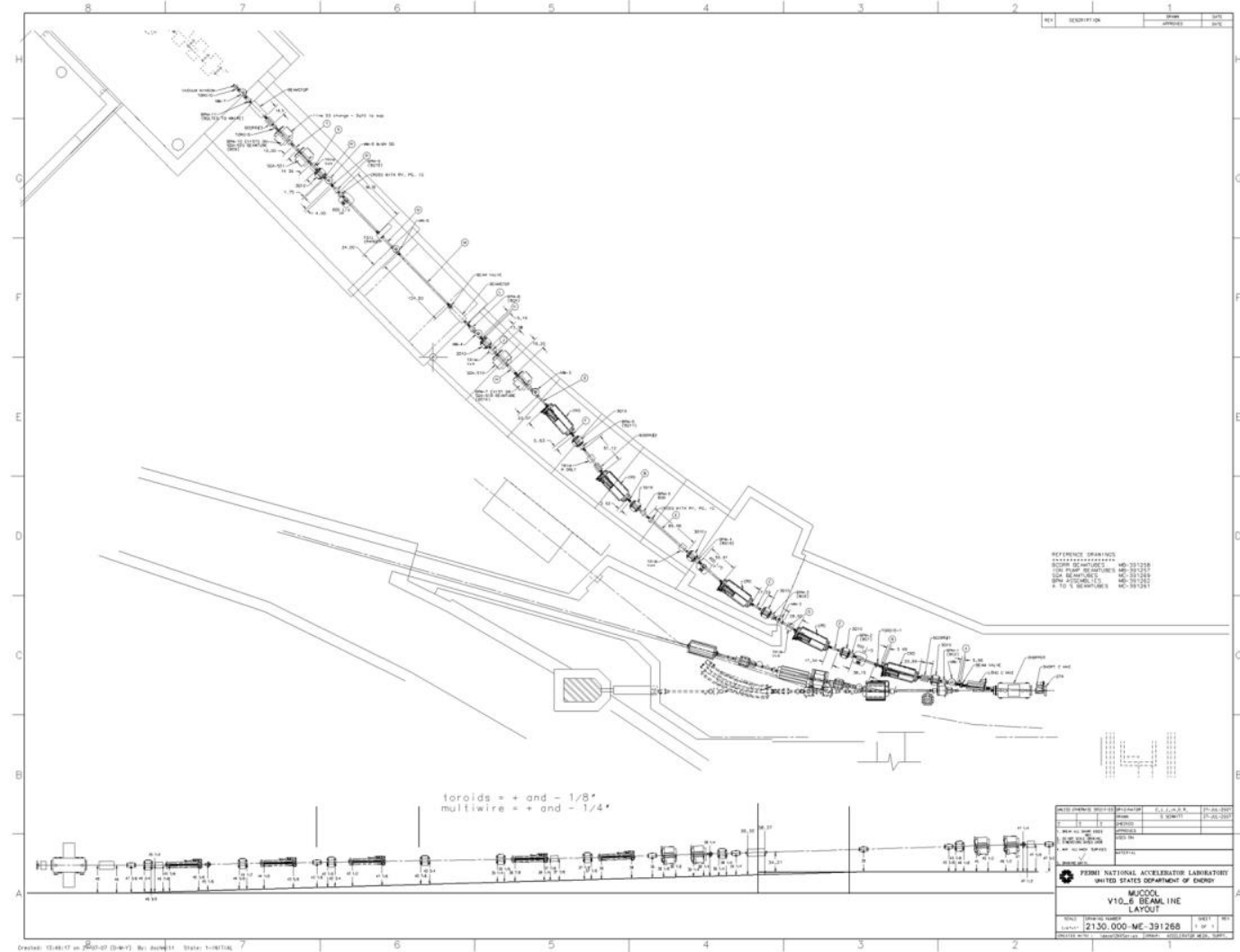

Figure 3.5: MTA proton beamline layout. 


\begin{tabular}{|c|c|c|c|}
\hline \multicolumn{4}{|l|}{$\begin{array}{l}\text { MTA TRANSFER LINE } \\
\text { Primary Parameters }\end{array}$} \\
\hline Beam kinetic energy & 400 & \multicolumn{2}{|l|}{$\mathrm{MeV}$} \\
\hline Particle type & \multicolumn{3}{|l|}{$\overline{\mathrm{H}^{-}}$} \\
\hline Peak current & 52 & \multicolumn{2}{|l|}{$\mathrm{mA}$} \\
\hline RF structure & 201.24 & \multicolumn{2}{|l|}{$\mathrm{MHz}$} \\
\hline Range repetition rate & 1 & 15 & $\mathrm{~Hz}$ \\
\hline Beam width & 2 & \multicolumn{2}{|l|}{50} \\
\hline Particle per pulse & 0.6 & 16 & $10^{12}$ \\
\hline Average beam current & 0.10 & 40 & $\mu \mathrm{A}$ \\
\hline Beam power & 0.04 & 15 & KW \\
\hline Transfer line length to Experimental Hall & 45 & $\mathrm{~m}$ & incl shielding wall \\
\hline Transfer line total bend & \multicolumn{2}{|l|}{$45^{\circ}$} & horizontal \\
\hline $\begin{array}{l}\beta_{\text {Man }} \mathrm{H}(\mathrm{V}) \\
\beta_{\text {Min }} \mathrm{H}(\mathrm{V}) \\
\end{array}$ & \multicolumn{2}{|l|}{$130(110)$} & diagnostic mode \\
\hline $\begin{array}{l}\beta_{\text {Maz }} \mathrm{H}(\mathrm{V}) \\
\beta_{\text {Min }} \mathrm{H}(\mathrm{V}) \\
\end{array}$ & $\begin{array}{r}40(30) \\
\approx 0.5 \\
\end{array}$ & $\begin{array}{l}\mathrm{m} \\
\mathrm{m}\end{array}$ & MTA mode \\
\hline Dispersion at entrance(exit) & $0(2)$ & \multicolumn{2}{|l|}{$\mathrm{m}$} \\
\hline Dispersion Max. & 7 & \multicolumn{2}{|l|}{$\mathrm{m}$} \\
\hline \multicolumn{4}{|l|}{ Active Elememts } \\
\hline \multirow[t]{4}{*}{ Quadrupoles in transfer line } & \multicolumn{2}{|l|}{12} & \multirow[b]{2}{*}{ Gradient (T/m) } \\
\hline & Quantity & Length $(\mathrm{m})$ & \\
\hline & 8 & 0.245 & 11.8 \\
\hline & 4 & 0.423 & 9.5 \\
\hline \multicolumn{4}{|l|}{ Dipoles in transfer line } \\
\hline Horizontal Bending Cooling Ring & 5 & \\
\hline Dipole physical length & 1.219 & \multicolumn{2}{|l|}{$\mathrm{m}$} \\
\hline Dipole gap & 0.083 & \multicolumn{2}{|l|}{$\mathrm{m}$} \\
\hline Dipole Field & 0.43 & \multicolumn{2}{|l|}{$\mathrm{T}$} \\
\hline Bend angle & $10^{\circ}$ & & \\
\hline Conductor Current & 711 & Amps & \\
\hline Steering Trim Magnets & 8 & & \\
\hline Vertical Trim Magnets & 4 & air cooled 0 . & kG-m/Amps \\
\hline Horizontal Trim Magnets & 4 & air cooled 0. & kG-m/Amps \\
\hline Instrumentation & & & \\
\hline Beam Position Monitors & 10 & & \\
\hline Multi Wires & 7 & & \\
\hline Toroids & 3 & & \\
\hline Beam Loss Monitors & 12 & & \\
\hline Vacuum & & & \\
\hline Vacuum required & $10^{-7}$ & torr & \\
\hline Ion pumps & 3 & & \\
\hline Turbo Cart & 1 & & \\
\hline Vacuum Valves & 2 & & \\
\hline
\end{tabular}

The majority of the beamline components are from formerly operational areas at Fermilab, and have been refurbished and refitted. In addition, there are four new elements: two identical beam stops and two extraction magnets (C-magnets). The extraction magnets and beam stops have been built, and the beam stops were installed during the summer shutdown. Instrumentation and vacuum elements as well as utility systems have also been installed. To complete the beamline, the extraction magnets, and a few additional diagnostics and spool pieces must be installed. In addition, the MTA radiation mitigation must be enhanced in three areas: (i) a radiation fence must be added around the berm, and a hardware limit implemented on the repetition rate of the $\mathrm{C}$ - 
magnet power supply, (ii) in the hatch area (which contains shielding blocks and the RF penetration) the RF must be reconfigured and shielding added, and (iii) configuration control must be imposed in the MTA experimental hall. The shielding wall has already been unstacked and reconfigured to fulfill the radiation shielding requirements. This reconfiguration has, from the radiation standpoint, decoupled the MTA from the Linac, allowing shielding work to continue in the MTA after the shutdown without impacting Linac operations.

\subsubsection{HPRF Beam Test Cavity}

The HPRF beam tests will require a new test cavity. The design and constructed of this new cavity is being supported by Muons Inc. SBIR funding. One possible design, shown in Fig. 3.6, consists of three chambers. The largest chamber (green wall) is a cryogenic container which contains liquid nitrogen (or air for room temperature tests). The middle chamber (red wall) is a pressurized vessel. This is designed for the same pressure range as the current TC cavity (1600 PSIA), and can also be evacuated $\left(10^{-7} \sim 10^{-8}\right.$ Torr) to facilitate a comparison of HP and vacuum operation. The smallest chamber (blue wall) is a resonant cavity which can be tuned by changing the distance between upstream and downstream plates. This motion is generated by applying a pressure difference between the middle and the smallest chambers. Once the pressure in both chambers is equal (equilibrium) the wall is fixed. The final design will be completed later this year.

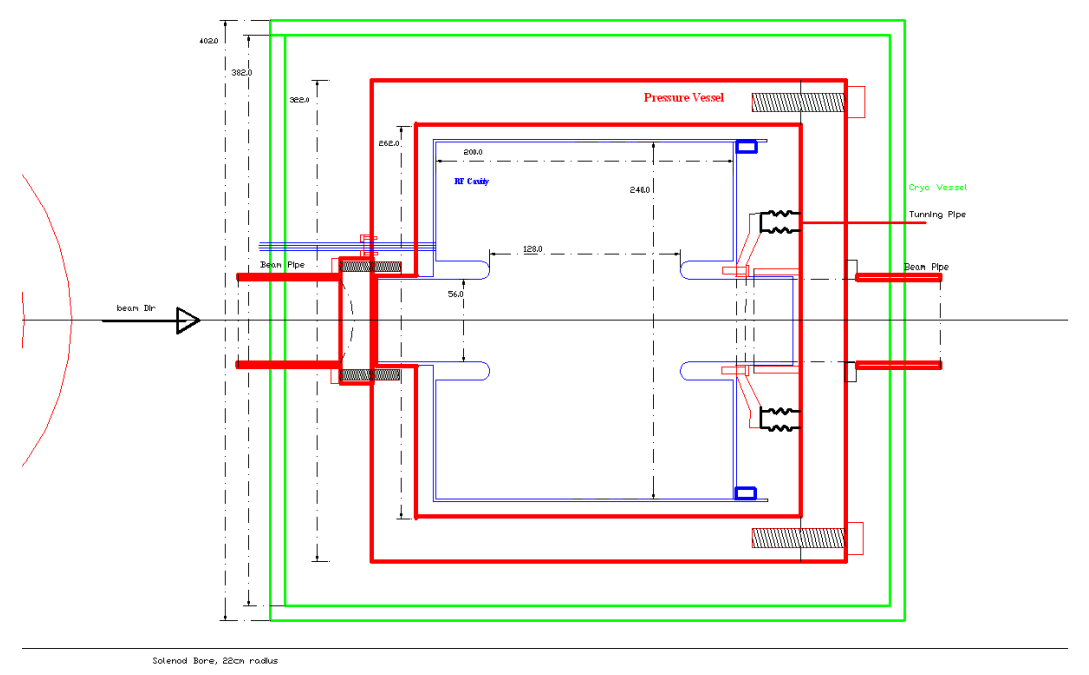

Figure 3.6: Schematic draw of a new test cell cavity.

In the current run plan, the HPRF cavity will be exposed to $\sim 6 \times 10^{12}$ protons in a $22 \mu \mathrm{s}$ long pulse with a repetition rate of the order of a minute. A MARS calculation of the radiation fluence in and near the cavity is shown in Fig. 3.7. Almost all of the simulated incoming $400 \mathrm{MeV}$ protons make it into the electrode gap, with a kinetic energy loss of $150 \sim 200 \mathrm{MeV}$ in the upstream stainless plate. The calculated number of delta electrons (with kinetic energy more than $1 \mathrm{keV}$ ) in the gap is $\sim 70$ per $250 \mathrm{MeV}$ proton in $400 \mathrm{~atm}$ pressurized hydrogen gas. Therefore, a total of $10^{13} \sim 10^{14}$ charged particles are expected to exist in the gap per pulse, which is comparable to the number of muons in a Muon Collider ionization cooling channel. Once beam is available at the MTA we plan to 
measure the breakdown field as a function of pressure in the presence of the ionizing beam. The detailed run plan will be formulated in the coming weeks.

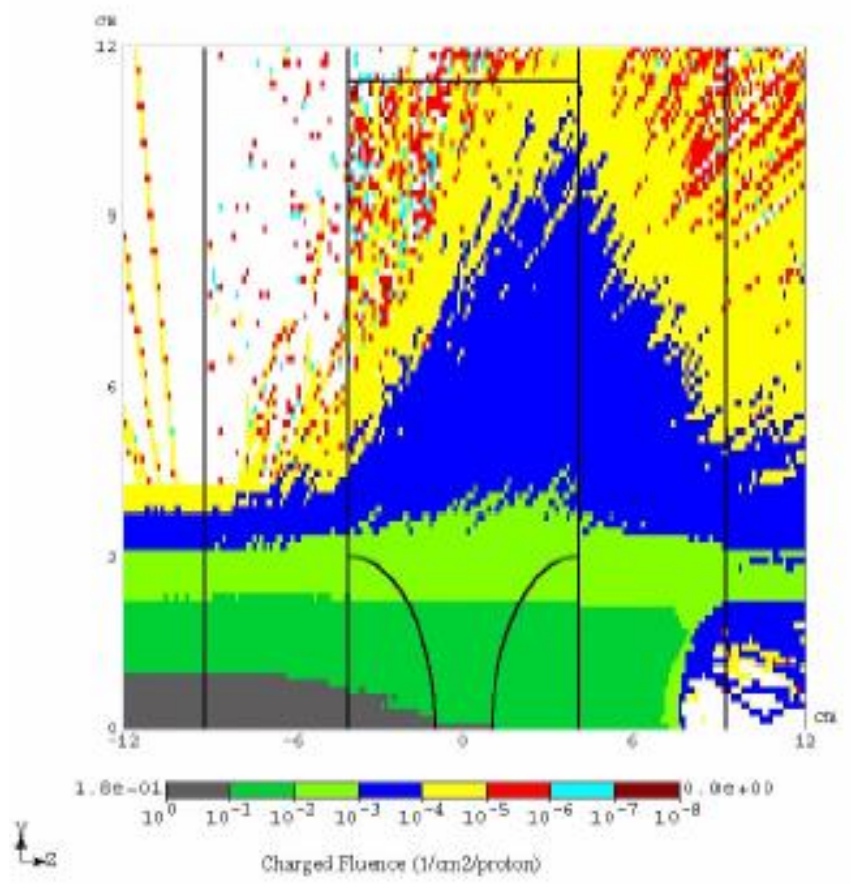

Figure 3.7: Simulated radiation strength in the TC by injecting a 400MeV proton beam (from left side).

\section{Helical Cooling Channels}

A significant six-dimensional (6D) phase space cooling of a muon beam in a short time is an essential requirement for muon colliders. Ionization cooling is the only cooling method fast enough to act in the short lifetime of the muons. The total amount of cooling that can be achieved is given by the amount of absorber placed in the beam path, which in turn is limited by the amount of RF voltage available to restore the lost longitudinal momentum. In order to maximize the efficiency of the cooling channel, one would therefore like to pack absorber and RF cavities as closely as possible, which is one reason pressurized cavities are so desirable, since both absorber and RF share the same space.

Ionization cooling is inherently transverse, so in order to cool longitudinally one needs to introduce a correlation between muon momentum and path length in the absorber. In the early schemes, this was done by using absorber wedges in dispersive regions. More recently, it has been proposed to use a continuous absorber and instead rely on the field configuration in a helical magnet to generate the path length difference. This scheme has been called a Helical Cooling Channel (HCC). The helical magnet combines solenoid field components with helical dipole, quadrupole and sextupole field components. It produces a continuous dispersion, which generates a continuous emittance exchange between transverse and longitudinal phase spaces via an interaction with the absorber. Pressurized hydrogen filled RF cavities are used in the HCC to both provide the absorber 
and compensate the energy loss. Because of the compactness, the HCC promises efficient cooling properties.

\subsection{Helical Cooling Channel Theory and Simulations}

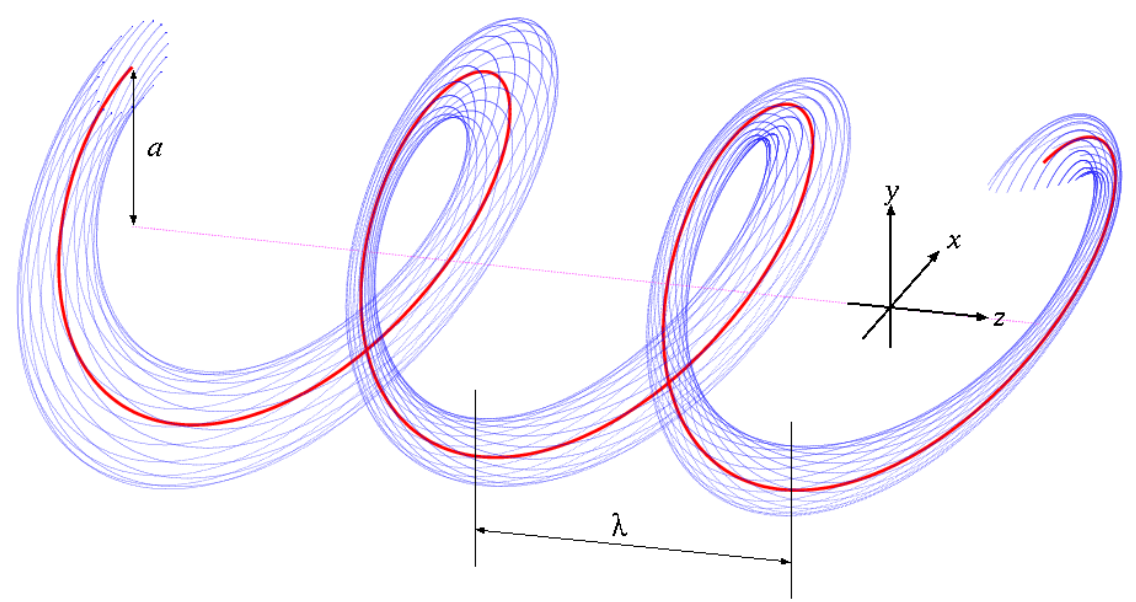

Figure 4.1.1: Particle motion in the helical magnet. The conductor of the helical magnet is invisible.

Figure 4.1.1 shows the simulated particle tracks in a helical magnet without any absorbers. The radius of the reference orbit from the axial center of the magnet is given by $a$ while $\lambda$ represents the helical orbit period. The amplitudes the field components ( $B$ solenoid, $b$ helical dipole, $b$ ' helical quadrupole) on the reference orbit can be expressed in terms of the tangential pitch $\kappa=2 \pi a / \lambda\left(=\mathrm{p}_{\varphi} / \mathrm{p}_{\mathrm{z}}\right)$ and the helical wave number $k(=$ $2 \pi / \lambda)$ as $\left[{ }^{21}, 22,23,24\right]$

$$
\begin{aligned}
& B=\frac{p k(1+q)}{\sqrt{1+\kappa^{2}}} \\
& b=\frac{\kappa}{1+\kappa^{2}} \frac{q}{(1+q)} B \\
& b^{\prime}=-\frac{g p k^{2}}{\left(1+\kappa^{2}\right)^{3 / 2}}
\end{aligned}
$$

where $p$ is the magnetic rigidity of the muons. The field index $g$ and the relative difference $q$ between the Larmor oscillation wave number and the helical orbit wave number are given by,

$$
\begin{gathered}
q=\sqrt{\frac{1+\kappa^{2}-1 / 2 \kappa \hat{D}}{1+1 / 2 \kappa^{2} /\left(1+\kappa^{2}\right)}} \\
g=\hat{D}^{-1}-\frac{\kappa+\left(1+\kappa^{2}\right) q}{1+\kappa^{2}}
\end{gathered}
$$

where $\hat{D}=\frac{p}{a} \frac{d a}{d p}$ is a dispersion function. By tuning the dispersion function, the cooling properties of the helical channel can be modified. For example, the linear theory predicts that equal cooling decrements in the transverse and longitudinal planes can be obtained by choosing the dispersion 


$$
\hat{D}=2 \frac{1+\kappa^{2}}{\kappa^{2}}\left(1+\frac{2}{3} \beta^{2}\right)
$$

where $\beta$ is the Lorenz velocity parameter (In practice, for low momentum muons this expression needs to be modified to account for the energy dependence of $\mathrm{dE} / \mathrm{dx}$ ). To increase the acceptance of the cooling channel, a helical sextupole term

$$
b^{\prime \prime}=-\frac{b^{\prime}}{2 \hat{D}}
$$

can be added to compensate for chromatic aberrations.

The helical cooling channel has been modeled using G4BeamLine, which is based on Geant4. Simulations are typically performed starting with a Gaussian muon beam that is significantly larger than the acceptance. Each individual muon is tracked through the HCC structure, and the emittance evolution is then calculated based on only those tracks that make it all the way to the end. In this way, ionization cooling can be distinguished from evaporative cooling (i.e. scraping of high amplitude particles). The resulting emittance evolution has a certain degree of dependence on the initial beam distribution, but it can be argued that the dependence is small, in particular if the initial beam is very large so that the distribution of particles inside the acceptance is close to uniform.

The 6D cooling decrements predicted from the helical cooling theory have been verified by simulations $\left[{ }^{22}\right]$, and further numerical optimization of the field configuration has extended the stability region of the beam phase space $\left[{ }^{23},{ }^{24}\right]$. Figure 4.1 .2 shows the emittance evolution in a series of four HCC segments. Here, the helical magnetic field is generated by ideal helical current conductors. The pressure of the gaseous hydrogen absorber in the RF cavities is 400 atm at room temperature. $\kappa$ is 1.0 in the whole channel to simplify the connection between HCC sections. The obtained 6D cooling factor is more than 50,000 in 160 meters. The cooling performance quoted here, and in the rest of section, is given in terms of rms normalized emittance.

\subsection{Helical Magnet Design}

The magnet design work has been focused on producing a section of HCC magnet that can be tested without $\mathrm{RF}\left[{ }^{25},{ }^{26}\right]$. In order to do this, the magnetic fields must be tapered to follow the muon momentum decrease in the absorber. Two conceptually different versions of the magnet system have been investigated. The first is the large bore "conventional" design which consists of a solenoid coil with separate helical dipole and quadrupole coils wrapped around it. The second is a small bore "helical solenoid". In this case, the magnet consists of many individual solenoid coils arranged in a helical pattern. The helical dipole and quadrupole components are generated by the coil offsets and radii. As will be discussed later, the helical solenoid version introduces some design constraints, but is significantly easier and cheaper to build. Table 4.2.1 summarizes the parameters of the designs considered. $\mathrm{B}_{\tau}$ is the tangential field component and $\mathrm{B}_{\mathrm{z}}$ is the longitudinal field component. The solenoid field component was not considered in this study due to its small effect on the beam cooling factor. 


\begin{tabular}{|l|l|l|l|}
\hline$\lambda=1.0 \mathrm{~m}$ & $\lambda=0.8 \mathrm{~m}$ & $\lambda=0.6 \mathrm{~m} \quad \lambda=0.4 \mathrm{~m}$ \\
\hline
\end{tabular}
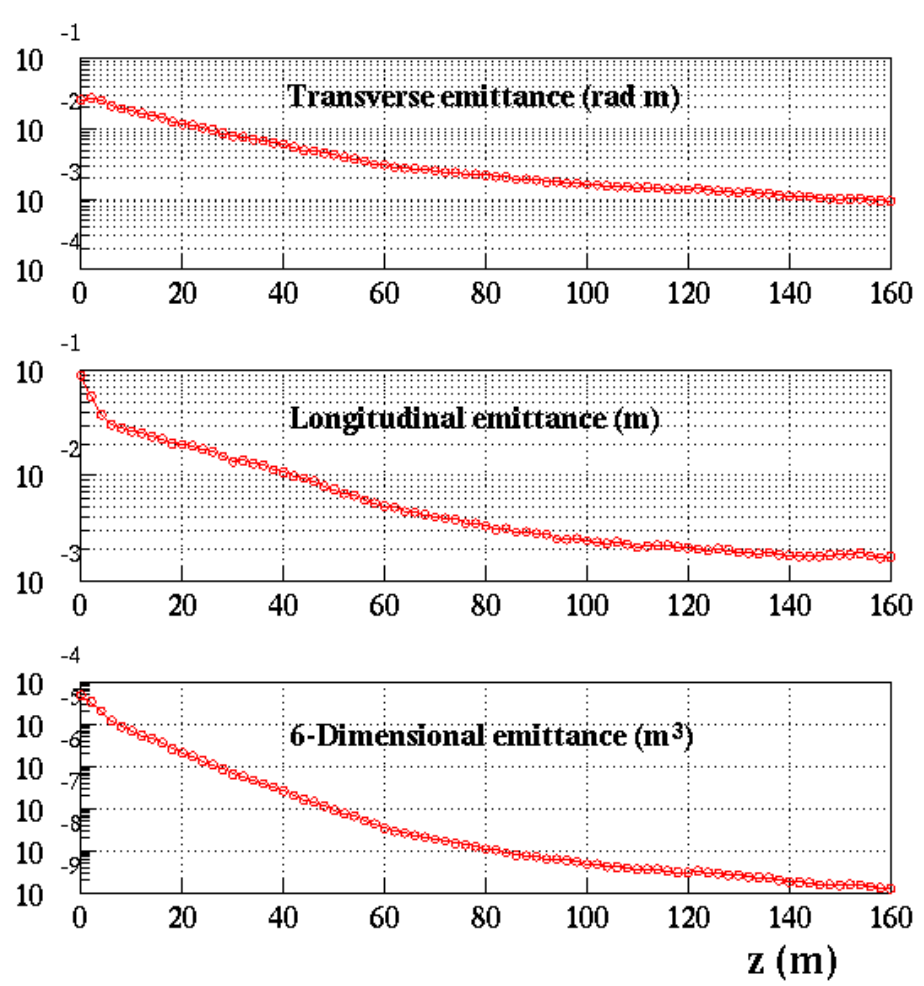

1st HCC: Total Length $=50 \mathrm{~m}, \lambda=1.0 \mathrm{~m}, \kappa=1.0$

$\mathrm{B}=-6.95 \mathrm{~T}$

$\mathrm{b}=1.62 \mathrm{~T}$

$\mathrm{b}^{\prime}=-0.72 \mathrm{~T} / \mathrm{m}$

$\mathrm{b}^{\prime \prime}=0.20 \mathrm{~T} / \mathrm{m}^{2}$

$\mathrm{RF}$ frequency $=200 \mathrm{MHz}$

$\mathrm{E}=31.5 \mathrm{MV} / \mathrm{m}$

$\varphi \mathrm{rf}=140$ degree

2nd HCC: Total Length $=30 \mathrm{~m}, \lambda=0.8 \mathrm{~m}, \kappa=1.0$

$\mathrm{B}=-8.69 \mathrm{~T}$

$\mathrm{b}=2.03 \mathrm{~T}$

$b^{\prime}=-1.12 \mathrm{~T} / \mathrm{m}$

$\mathrm{b}^{\prime \prime}=0.32 \mathrm{~T} / \mathrm{m}^{2}$

$\mathrm{RF}$ frequency $=200 \mathrm{MHz}$

$\mathrm{E}=31.5 \mathrm{MV} / \mathrm{m}$

prf $=140$ degree

3rd HCC: Total Length $=30 \mathrm{~m}, \lambda=0.6 \mathrm{~m}, \mathrm{\kappa}=1.0$

$\mathrm{B}=-11.6 \mathrm{~T}$

$\mathrm{b}=2.71 \mathrm{~T}$

$\mathrm{b}^{\prime}=-2.00 \mathrm{~T} / \mathrm{m}$

$\mathrm{b}^{\prime \prime}=0.56 \mathrm{~T} / \mathrm{m}^{2}$

$\mathrm{RF}$ frequency $=200 \mathrm{MHz}$

$\mathrm{E}=31.5 \mathrm{MV} / \mathrm{m}$

prf $=140$ degree

4th HCC: Total Length $=40 \mathrm{~m}, \lambda=0.4 \mathrm{~m}, \kappa=1.0$

$\mathrm{B}=-17.4 \mathrm{~T}$

$\mathrm{b}=4.06 \mathrm{~T}$

$\mathrm{b}^{\prime}=-4.49 \mathrm{~T} / \mathrm{m}$

$\mathrm{b}^{\prime \prime}=1.26 \mathrm{~T} / \mathrm{m}^{2}$

$\mathrm{RF}$ frequency $=200 \mathrm{MHz}$

$\mathrm{E}=31.5 \mathrm{MV} / \mathrm{m}$

prf $=140$ degree

Figure 4.1.2: Beam emittance evolution in a series of helical cooling channels. The gaseous hydrogen pressure is 400 atm at room temperature in this simulation.

Table 4.2.1: HCC magnet reference parameters

\begin{tabular}{lccc}
\hline \hline \multicolumn{1}{c}{ Parameter } & Unit & Large bore & Small bore \\
\hline Length of the helical section & $\mathrm{m}$ & 4.0 & 3.2 \\
Helix twist pitch & $\mathrm{m}$ & 2.0 & 1.6 \\
Radius of the reference orbit & $\mathrm{m}$ & 0.25 & 0.25 \\
Initial $\mathrm{B}_{\tau}$ & $\mathrm{T}$ & 1.045 & 1.249 \\
$\partial \mathrm{B}_{\tau} / \partial \mathrm{z}$ & $\mathrm{T} / \mathrm{m}$ & -0.133 & -0.170 \\
$\mathrm{Initial}_{2} \partial \mathrm{B}_{\tau} / \partial \mathrm{r}$ & $\mathrm{T} / \mathrm{m}$ & 0.603 & -0.882 \\
$\partial^{2} \mathrm{~B}_{\tau} / \partial \mathrm{r} / \partial \mathrm{z}$ & $\mathrm{T} / \mathrm{m}^{2}$ & -0.052 & 0.069 \\
Initial & $\mathrm{T}$ & -3.753 & -3.859 \\
$\partial \mathrm{B}_{\mathrm{z}} / \partial \mathrm{z}$ & $\mathrm{T} / \mathrm{m}$ & 0.467 & 0.544 \\
\hline \hline
\end{tabular}

\subsubsection{Large Bore Cooling Channel}

The relatively small field in the cooling channel suggests use of well-known $\mathrm{NbTi}$ technology, and $\mathrm{NbTi}$ superconducting wire with $\mathrm{J}_{\mathrm{c}}(5 \mathrm{~T}, 4.2 \mathrm{~K})=3000 \mathrm{~A} / \mathrm{mm}^{2}$ has therefore been used in the design. To provide good field quality over the desired length, one bore radius has been added to each end of the good field region, resulting in a helical section length of $5 \mathrm{~m}$ for the given channel. After comparison of different design 
approaches in terms of simplicity and efficiency, a layered design concept was developed. Each coil layer has a uniform cross-section and a constant current throughout the entire length. The upstream end of each layer is located at the same longitudinal coordinate, while the downstream end coordinate varies along the channel, providing the longitudinal gradient.

The helical dipole consists of six layers. The first layer has a $5 \mathrm{~m}$ long helical section, and in each following layer the helical section is shorter than the previous one by a halfperiod of the helix $(1 \mathrm{~m})$. The last layer has zero helical section length. The helical dipole coil was designed to match the $\mathrm{B}_{\tau}$ and $\partial \mathrm{B}_{\tau} / \partial \mathrm{z}$ components in Table 4.2.1, and produce the desired field on the beam orbit. In the present design the required helical dipole component and its longitudinal gradient are reproduced relatively well and the initial $\partial \mathrm{B}_{\tau} / \partial \mathrm{r}$ component is close to the target. The average longitudinal gradient $\partial^{2} \mathrm{~B}_{\tau} / \partial \mathrm{r} / \partial \mathrm{z}$ and $\mathrm{B}_{\mathrm{z}}$ component need further correction which can be achieved with the help of dedicated quadrupole and solenoid coils.

The eight-layer helical quadrupole coil is placed around the dipole coil. Since, at the upstream end, the transverse field quadrupole component produced by the dipole is close to the required value, the current in the quadrupole coil should start from zero at the upstream end which allows trimming the coil length. The first layer of the quadrupole coil has a $3.5 \mathrm{~m}$ long straight section and is shifted by $1 \mathrm{~m}$ towards the downstream end. Each following layer is shorter than the previous one by a quarter-period of the helix $(0.5$ $\mathrm{m}$ ), and the downstream end of each layer is located at the same longitudinal coordinate as the downstream end of the first layer. The average $\partial^{2} \mathrm{~B}_{\tau} / \partial \mathrm{r} / \partial \mathrm{z}$ component is close to the target. However, the helical coils produce $\mathrm{B}_{\mathrm{z}}$ and $\partial \mathrm{B}_{\mathrm{z}} / \partial \mathrm{z}$ components with the wrong signs. This has to be corrected by the main solenoid.

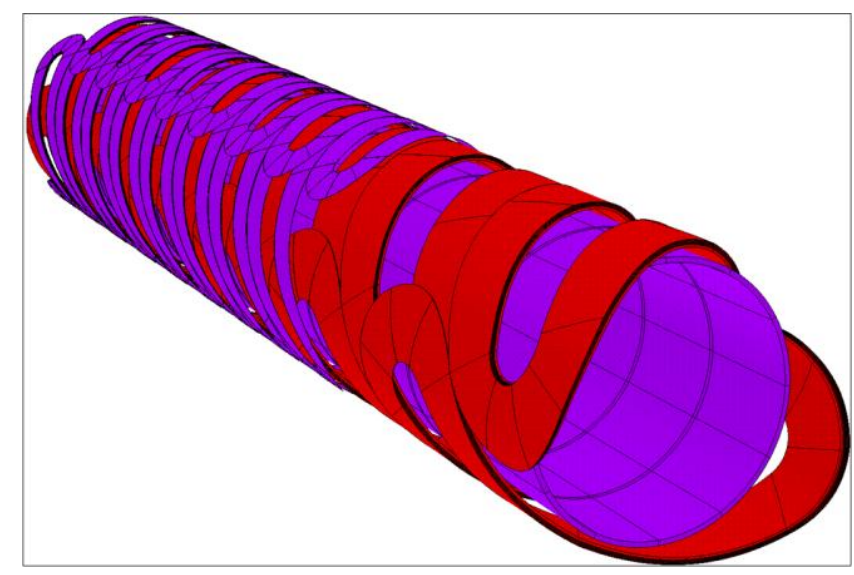

Figure 4.2.1: Large bore solenoid with helical dipole and quadrupole coils.

According to Table 4.2.1, the solenoid should produce the largest field component. To maximize the efficiency of the solenoid, and avoid unnecessary exposure of other coils to the strong longitudinal field, it makes sense to place it between the dipole winding and the bore tube. An extra two bore radii were added to the criterion mentioned earlier to compensate the field decay. The solenoid has a total length of $6 \mathrm{~m}$ and consists of 12 sections with a varying number of ampere-turns to generate the field gradient in the longitudinal direction. Figure 4.2.1 shows the helical dipole, quadrupole and solenoid 
coils and Fig. 4.2.2 their combined field. On average, all field components and their longitudinal gradients are close to the required values.

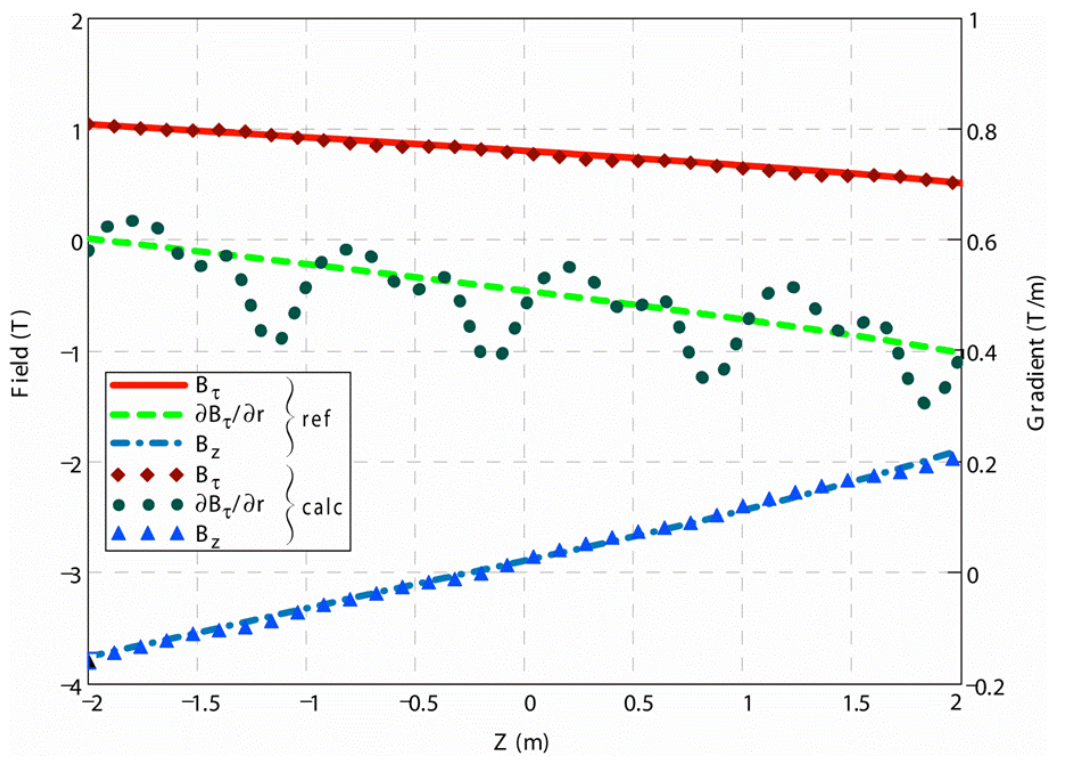

Figure 4.2.2: Large bore solenoid field distribution.

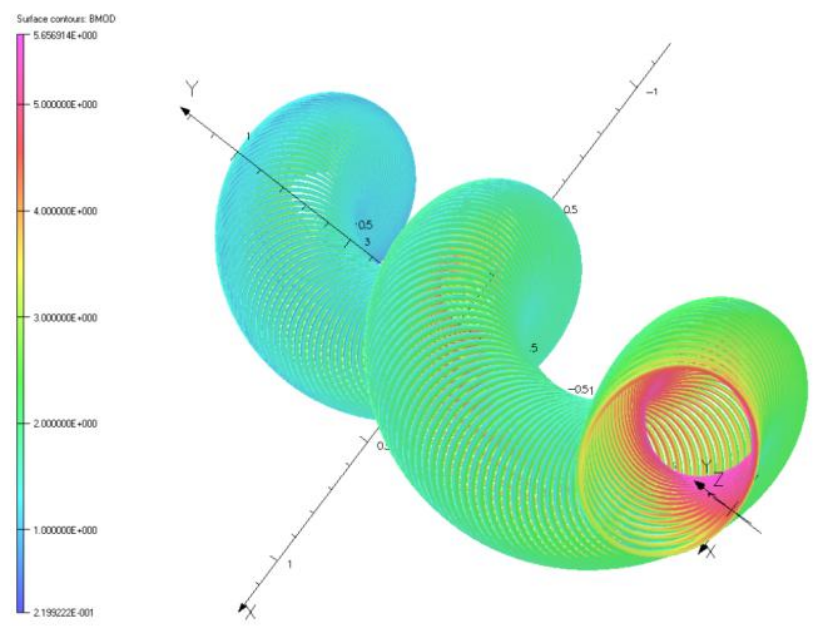

Figure 4.2.3: Helical Solenoid geometry

\subsubsection{Small Bore Cooling Channel}

A novel approach is to use a helical solenoid (HS) to generate the required field components. The basic concept is to use circular short coils shifted in the transverse direction to the $\mathrm{z}$ axis (Fig. 4.2.3). All coil centers lay on a helical beam orbit and are equally distributed along $\mathrm{z}$. Because the orbit is tilted relative to the coils, they simultaneously generate longitudinal and transverse field components. The current in the rings changes along the channel to obtain the longitudinal field gradients. In contrast to the large bore system, where the longitudinal and transverse field components are controlled by independent windings, the small bore system has a fixed relation between 
all components for a given set of geometrical constraints. Thus, to obtain the necessary cooling effect, the coil should be optimized together with the beam parameters.

Figure 4.2.4 shows the optimum initial transverse field gradient $\partial \mathrm{B}_{\tau} / \partial \mathrm{r}$ from the beam simulations and the one calculated in the helical solenoid as a function of helix period.

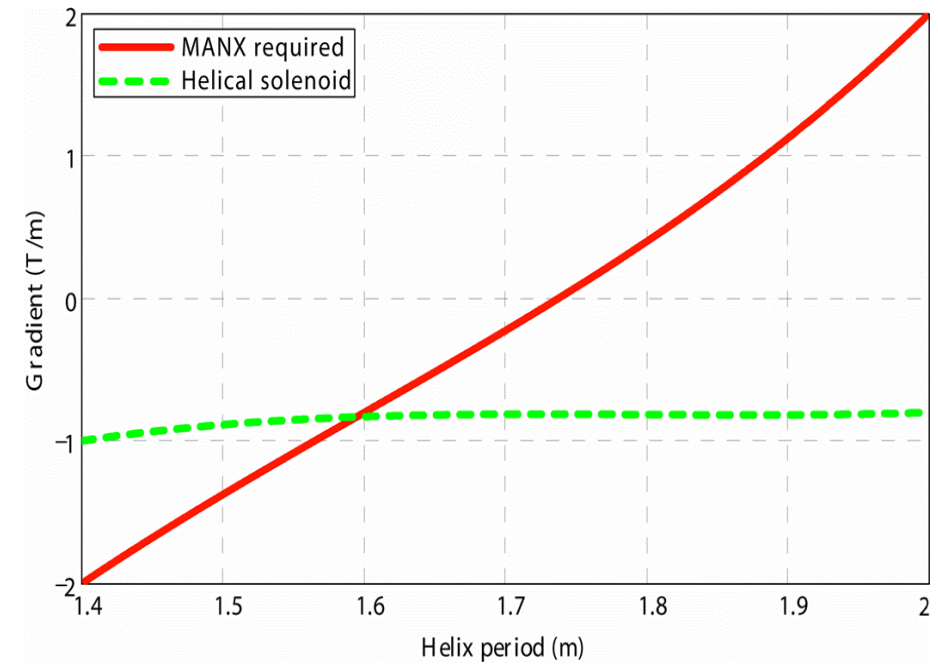

Figure 4.2.4: The transverse field gradient in Small bore helical solenoid.

The muon momentum is reduced from $300 \mathrm{MeV} / \mathrm{c}$ to $160 \mathrm{MeV} / \mathrm{c}$ in passing along the 5.6 $\mathrm{m}$ helical path through the LHe absorber, and the magnetic field strength must diminish with the momentum to provide a stable beam orbit. Magnetic field simulations were performed to investigate the behavior of the HS. Figure 4.2.5 shows the relative field components for a model in which the current in the coils was decreased linearly as a function of the longitudinal $\mathrm{z}$-coordinate with gradient $-13 \% / \mathrm{m}$. One can see the interesting result that the three important field components, (solenoidal (Bz), helical dipole $(\mathrm{Bt})$, and helical quadrupole $(\mathrm{Gz}))$, scale with coil current.

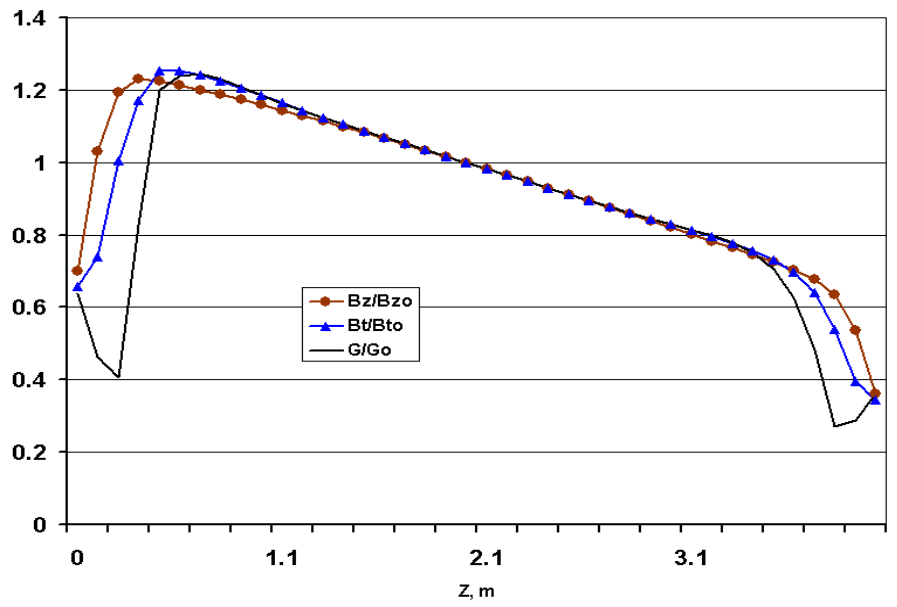

Figure 4.2.5: Field distribution related to the values at $z=2 m(B z o=-3.37 T, B t o=1.04 T$, $G o=-0.9 \mathrm{~T} / \mathrm{m})$. 
Figure 4.2.6 shows the dependence of the three field components as a function of coil radius. There is a linear dependence of field components and gradient with the coil radius change. At the same time $\mathrm{Gz}$ is constant and defined only by coil currents.

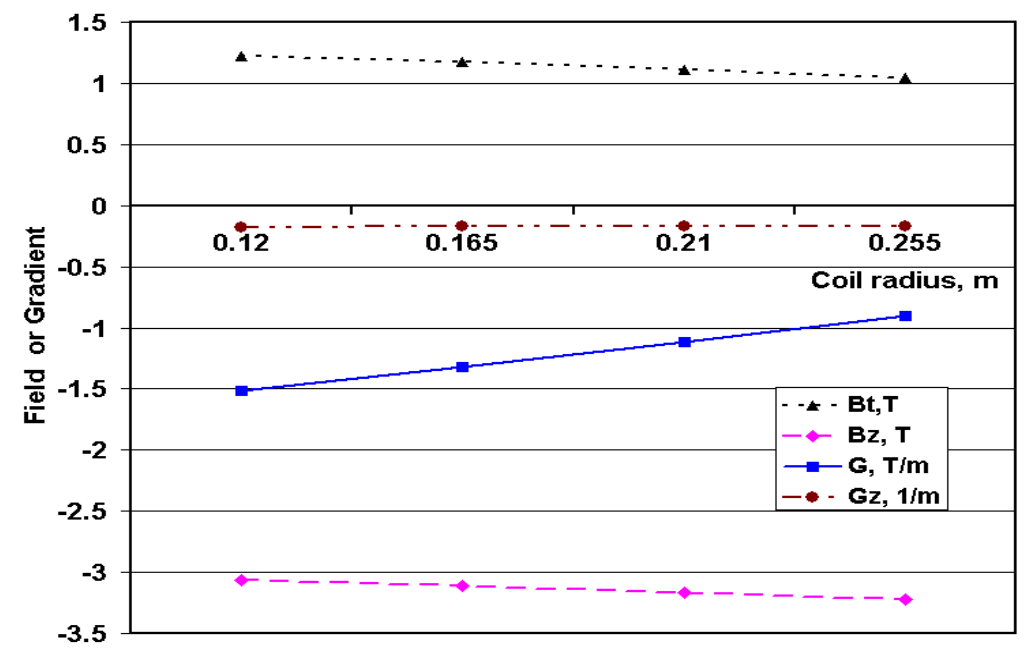

Figure 4.2.6: Field and field gradient dependence.

\subsubsection{Mechanics and Quench Protection}

The magnet systems generate 6-7 $\mathrm{T}$ fields in the coils. Large Lorentz forces in the solenoid should be intercepted by a strong support structure to provide the conductor mechanical stability. Forces applied to the dipole and quadrupole coils are smaller than in the solenoid because they are mounted outside of the solenoid in a region of lower field. The helical solenoid has more complicated forces and torques between the coils. The maximum forces are at the beginning of the cooling channel. The forces are both compressing the magnet in the longitudinal direction and acting to straighten the helical coil. The magnet systems store 5-30 MJ of energy in magnetic field and should have an active quench protection. In spite of the large stored energy, the sectioned design approach allows independent energy extraction from the coil sections which offers great flexibility in limiting voltages and temperatures. The relevant conductor parameters and system configuration will be determined during the quench protection analysis to limit the turn-to-turn and turn-to-ground voltages to $<1 \mathrm{kV}$ and coil hot spot temperature to $<300 \mathrm{~K}$.

\subsubsection{Baseline Magnetic System}

The advantages of the large bore system are flexibility in dealing with uncertainties of the beam travel through the cooling channel and independent adjustment of field parameters. The small bore system has the advantages of lower cost, mass, and stored energy, but it has a fixed relation between all field components. A short model and prototype fabrications and tests are planned to verify the magnet system performance. After an analysis of both magnet systems it was decided to proceed with the Helical Solenoid as the baseline version of magnet system. This system looks economically more promising and technically simpler although less Flexible, since the relative strength of the magnetic field components are set by the geometry rather than individual coil currents. 
As a first step toward building a helical solenoid magnet that can be tested in a beam, it is planned to build a subscale model with four solenoid coils. The geometry of the coils will be made to satisfy, as much as possible, the design requirements for the real magnet, while fitting within the Technical Division Vertical Magnet Test Facility (VMTF) Dewar. The mechanical support structure for the test coils will also simulate the geometry of the solenoids in the real magnet, again within the space constraints of the VMTF Dewar. The magnet will be tested in liquid helium, utilizing the full test capabilities of the Fermilab VMTF, and operated over a wide range of temperatures to fully test the Lorentz forces and test conductor. A magnetic measurement system will be developed to characterize the model magnet, validate the fields, and monitor field stability during current excitation.

Valuable knowledge will be gained in this test, which may be applied towards the design of the real magnet. First of all, the engineering design (i.e. tooling to make the coils + the manufacturing procedures) for the coils will be directly applicable to the full scale solenoid. The coil manufacturing process is a major time and cost driver for the full scale magnet and the test will thus reduce the uncertainty and required contingency in the final project. Similarly the mechanical support structures and the measurement system for the four coil test are directly applicable to the full scale magnet. The mechanical structure is dominated by the force from adjacent coils, with the end coils likely having the largest asymmetric forces. The magnetic system of this wide aperture magnet will be modular and will operating in liquid helium. Finally, the test coils can be used to do magnet studies that would be too costly or involve too much program risk for the full scale magnet. Studies include quench protection, complicated by strong field coupling of adjacent coils, and powering schemes for individual coils to compensate for required field variation due to $\mathrm{dE} / \mathrm{dx}$ loss. It is also possible to safely study the magnet response due to certain error conditions such as quench detection failure.

To fit within the Dewar, the coil diameter for the test model is $420 \mathrm{~mm}$ (compared to 510 $\mathrm{mm}$ for the present HCC design). The offset of coils matches the geometry for a $1.6 \mathrm{~m}$ helical solenoid period. Figure 4.2.7 shows the geometry of the support structure. Existing NbTi cable from the SSC inventory will be used. Tooling will be designed to wind the cable on a stainless steel mandrel. The cable will be insulated with polyimide tape, wound on "hard way bend". An outer retaining shell will restrain the coil both from hoop stress and longitudinal compression. The overall structure must hold the restoring forces that want to pull the coils onto a single solenoid axis. The coils will likely be potted for additional structural strength.

Table 4.2.2 shows the expected Lorentz forces during operation of the four coil Model magnet at nominal current, at peak field (maximum field, maximum stress), and a real helical solenoid magnet at nominal field. By testing at a higher current, the Lorentz forces on the structure can be made comparable to those expected in the real magnet.

Magnets tested in a vertical Dewar cryostat are typically tested using a "top hat", which serves as the room temperature interface of the helium volume. The magnet is hung from support rods from this top hat plate which also provides penetrations for power leads and instrumentation feed-throughs. An existing top hat built for VMTF will be used for the four-coil test. Modifications to the plate and mechanical interfaces will be required. As these coils have a very wide aperture the traditional magnetic measurement systems for 
accelerator magnets are not optimal, and therefore a new system, possibly an array of hall probes operating in liquid helium, will be used.

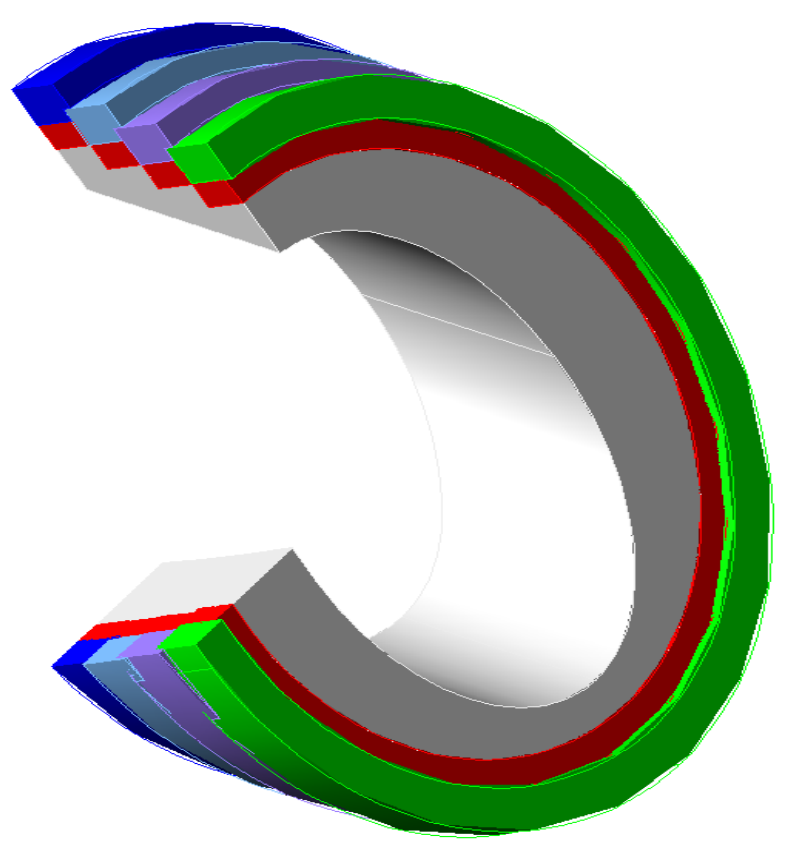

Figure 4.2.7: Cut-away view of the mechanical structure model magnet. Inner support (grey), superconducting coil (red) and outer collar rings (green-blue).

Table 4.2.2: Four Coil test parameters.

\begin{tabular}{|c|c|c|c|}
\hline Parameter & $\begin{array}{c}\text { Model } \\
\text { Nominal }\end{array}$ & $\begin{array}{c}\text { Model } \\
\text { Max }\end{array}$ & $\begin{array}{c}\text { Real } \\
\text { Nominal }\end{array}$ \\
\hline Peak superconductor field & $3.3 \mathrm{~T}$ & $4.84 \mathrm{~T}$ & $5.7 \mathrm{~T}$ \\
\hline Current & $9.6 \mathrm{kA}$ & $14 \mathrm{kA}$ & $9.6 \mathrm{kA}$ \\
\hline Number of turns/section & 10 & 10 & 10 \\
\hline Coil inner diameter & $420 \mathrm{~mm}$ & $420 \mathrm{~mm}$ & $510 \mathrm{~mm}$ \\
\hline Lorentz force/section, Fx & $70 \mathrm{kN}$ & $149 \mathrm{kN}$ & $160 \mathrm{kN}$ \\
\hline Lorentz force/section, Fy & $12 \mathrm{kN}$ & $25 \mathrm{kN}$ & $60 \mathrm{kN}$ \\
\hline Lorentz force/section, $\mathrm{Fxy}$ & $71 \mathrm{kN}$ & $151 \mathrm{kN}$ & $171 \mathrm{kN}$ \\
\hline Lorentz force/section, $\mathrm{Fz}$ & $157 \mathrm{kN}$ & $337 \mathrm{kN}$ & $299 \mathrm{kN}$ \\
\hline
\end{tabular}


Tests will be performed in the Fermilab VMTF, using as much as possible the existing test infrastructure, such as power supplies, quench detection and extraction circuitry and helium refrigerator plant. The test program will take approximately 2 weeks, depending on the quench performance of the magnet, and will consist of operation of the coils at full field. Magnetic measurement will be performed to determine field quality. Strain gauges will likely be used to determine the mechanical stress of the coils and coil support structure.

\subsection{RF Cavities in Helical Solenoid Magnet}

The original HCC simulations used ideal RF fields without considering the physical size of the cavities. A key design issue for the HCC is how to incorporate RF cavities into the helical solenoid. Three ideas have been investigated:

- RF cavities completely inside each magnet coil.

- RF cavities in between the coils.

- HCC segments without RF interleaved with segments of RF only (possibly in a solenoid field).
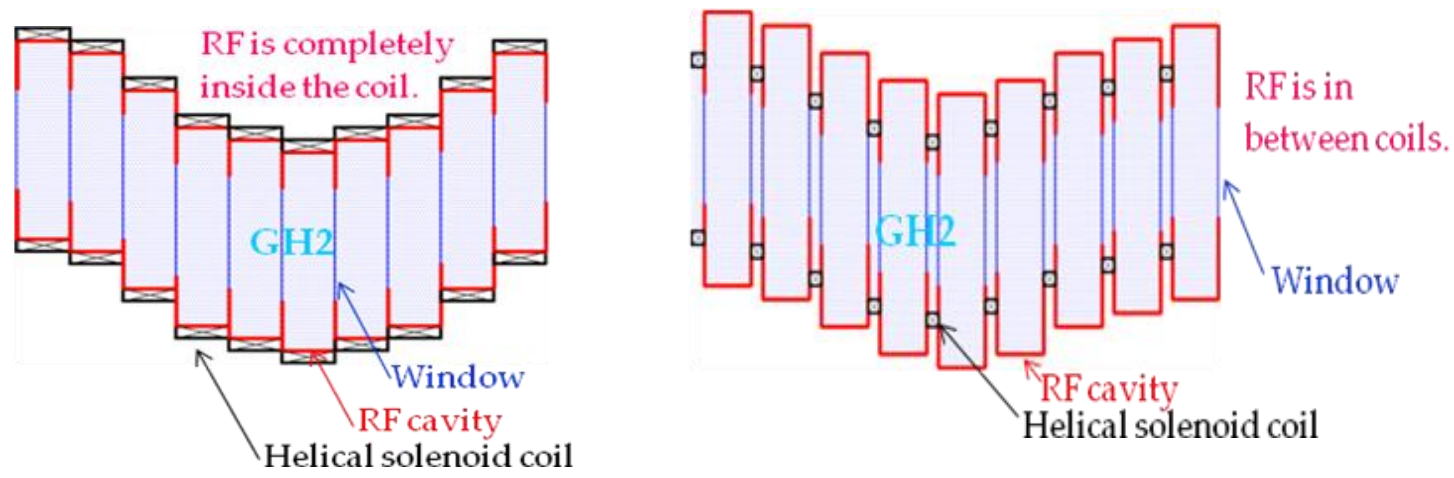

Figure 4.3.1: Conceptual pictures of simulation models. (Left) The RF cavity is mounted completely inside the helical coils (type1 model) and (Right) the RF cavity is mounted in between coils (type 2 model). The conductor coil is shown as a black box and the RF cavity as a red box in the figure. The beam window is shown in blue.

The two first models have been successfully studied in simulation, whereas the third has proven more difficult. Figure 4.3.1 shows schematics of the two simulation models which have the RF cavity in the helical solenoid coils. In the following, the scheme where the RF cavities are mounted completely inside the helical solenoid coils will be referred to as the 'type 1 model', while the scheme with the RF cavities mounted in between the helical solenoid coils will be referred to as the 'type 2 model'. Each cell has a thin window to create a closed cavity which is assumed to produce a perfect pillbox type electric field. This also means that the cavities must be coupled externally.

The type 1 model is quite similar to the original Muons Inc. HCC design [11], but uses a higher frequency RF in order to physically fit the cavity inside the coil. The relation 
between the resonant RF frequency in a pillbox cavity and the cavity size is given by, $f=$ $2.405 v / 2 \pi R$, where $v$ is the velocity of light in the material and $R$ is the radius of the resonant cavity, respectively. The velocity of light in 200 atm gaseous hydrogen is $0.95 \times$ $c$, thus the cavity radius is $5 \%$ smaller than the evacuated type cavity. The type 1 model has a strong geometric restriction on the cavity radius, and therefore the RF frequency. On the other hand, an advantage with the type 1 model is the magnetic field quality: the field map in the type 1 model almost perfectly matches the ideal field map because no big gap exists in between coils. On the other hand, there is no such geometric restriction in the type 2 model. The RF cavity can extend in the radial direction. Therefore, the resonant frequency may be arbitrary chosen. However, this model has a geometric limitation in the longitudinal direction. The gap between two coils must be limited from the point view of the helical field quality.

\subsubsection{Cavities inside coils}

Figure 4.3.2 shows the 6D emittance evolution in the type 1 model channel with various RF frequencies: 400, 800, and $1600 \mathrm{MHz}$. Table 4.3.1 shows the geometry and required field strength in the $\mathrm{HCC}$ with each frequency. In this simulation, to speed up the simulation time, the field map is produced using ideal helical current conductors, and no RF windows are included. The optimum gaseous pressure for the channel is $200 \mathrm{~atm}$. A denser gas destroys the stability of the longitudinal phase space with the higher frequency cavities.

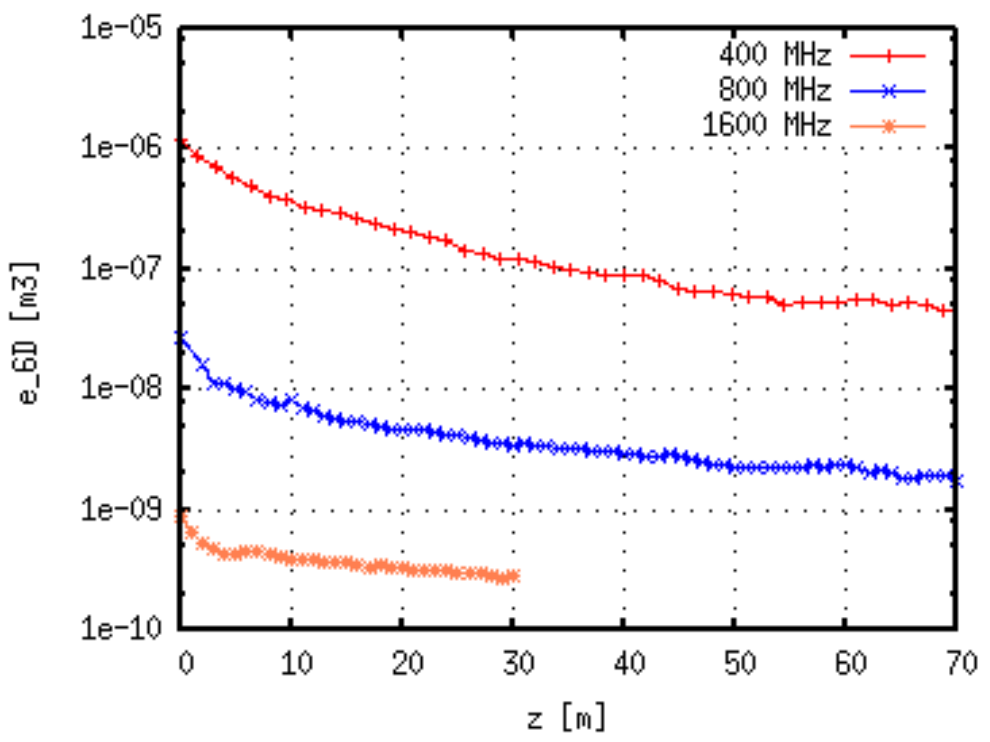

Figure 4.3.2: Cooling test in type1 model with various frequency RF fields

The HCC works well with $400 \mathrm{MHz}$ and $800 \mathrm{MHz}$ RF cavities, while the final emittance with $1600 \mathrm{MHz}$ cavities seems to be close to the equilibrium emittance. The acceptance of the HCC in higher frequency RF cavity is smaller than expected. The expected acceptance reduction in a double frequency cavity is simply $2 \times 2 \times 2=8$, while the simulation result show a reduction of $30 \sim 40$. From analysis of the transfer matrix, the reduced acceptance can result from a reduction in the angular momentum phase space, which may be attributed to the transverse variation of the RF gradient in the pill-box cavity. 


\begin{tabular}{|c|c|c|c|c|c|c|c|c|c|}
\hline Parameters & $\lambda$ & $\kappa$ & $B$ & $B$ & $B^{\prime}$ & $b^{\prime}$, & $\begin{array}{c}\text { Inner d } \\
\text { of coil }\end{array}$ & $\begin{array}{c}\text { EF } \\
\text { phase }\end{array}$ \\
\hline Unit & $\mathrm{M}$ & & $\mathrm{T}$ & $\mathrm{T}$ & $\mathrm{T} / \mathrm{m}$ & $\mathrm{T} / \mathrm{m}^{2}$ & $\mathrm{~cm}$ & $\mathrm{MV} / \mathrm{m}$ & Degree \\
\hline $400 \mathrm{MHz} \mathrm{HCC}$ & 1.6 & 1.0 & -4.3 & 1.0 & -0.28 & 0.08 & 50.0 & 16.4 & 140.0 \\
\hline $800 \mathrm{MHz} \mathrm{HCC}$ & 1.0 & 1.0 & -6.9 & 1.6 & -0.72 & 0.20 & 30.0 & 16.4 & 140.0 \\
\hline $1600 \mathrm{MHz} \mathrm{HCC}$ & 0.5 & 1.0 & -13.9 & 3.2 & -2.88 & 0.81 & 15.0 & 16.4 & 140.0 \\
\hline
\end{tabular}

\subsubsection{RF cavities between coils}

To fit RF cavities in between coils, a significant longitudinal space must be made available. This can be achieved by making the coils thinner and/or increasing their separation. In either case, the field quality is degraded. However, studies indicate that making the coils thinner does not significantly affect cooling performance, whilst increasing the separation between the coils by using fewer coils per helix period has a large impact on the quadrupole field component. It may be possible to compensate for this by readjusting the coil diameter.
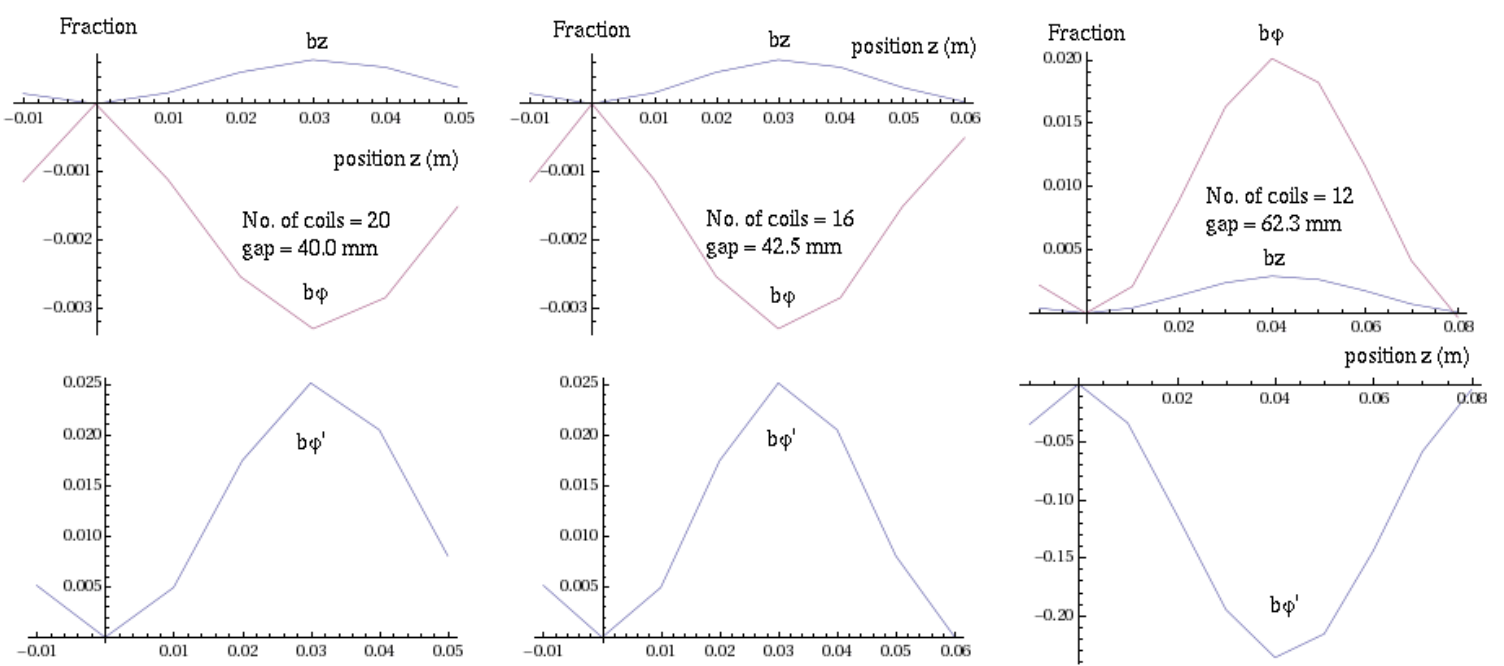

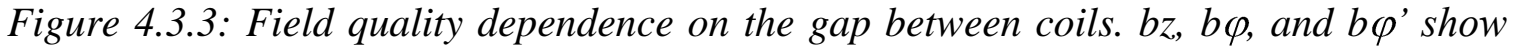
the fraction of amplitudes in solenoid, helical dipole, and helical quadrupole components, respectively.

Figure 4.3.3 shows the amplitude variation of the solenoid, helical dipole, and helical quadrupole components along the beam trajectory, for three different coil densities: 20, 16, and 12 coils per helix period. The variation of the solenoid and helical dipole amplitudes are generally small, of the order a few percent with the exception of the helical quadrupole component in the case of 12 coils per period. The variation of the transverse components also has the opposite sign compared to the higher coil density cases. This may be understood by noting that the coil displacement is comparable to the coil diameter. The average effect of the coil density on the field components may be canceled by adjusting the field strength and diameter of the individual solenoid coils. It 
has not yet been investigated whether the field fluctuations have a significant effect on cooling performance or dynamic aperture.

Figure 4.3.4 shows cooling simulation results for different coils lengths. The helical period is 1 meter, the gaseous pressure is $200 \mathrm{~atm}$, and the RF frequency is $400 \mathrm{MHz}$. There is no window in this simulation. For an $80 \%$ RF occupancy, the cavity length is 40 $\mathrm{mm}$ and the coil length is $10 \mathrm{~mm}$, while for a $40 \%$ RF occupancy, the cavity length is 20 $\mathrm{mm}$ and the coil length is $30 \mathrm{~mm}$. Naturally, the field variations in the $40 \% \mathrm{RF}$ occupancy channel are better than that in the $80 \%$ channel. The acceptance in the $80 \%$ occupancy channel is better than in the $40 \%$ case. This means that the synchrotron motion of the beam in the $80 \%$ occupancy channel is more stable than that in the $40 \%$ channel. There is no big difference in the cooling decrements between those channels. The conclusion is that the geometric restriction in the radial direction is more stringent than that in the longitudinal direction, and the longer RF cavity is preferable to stabilize the longitudinal motion.

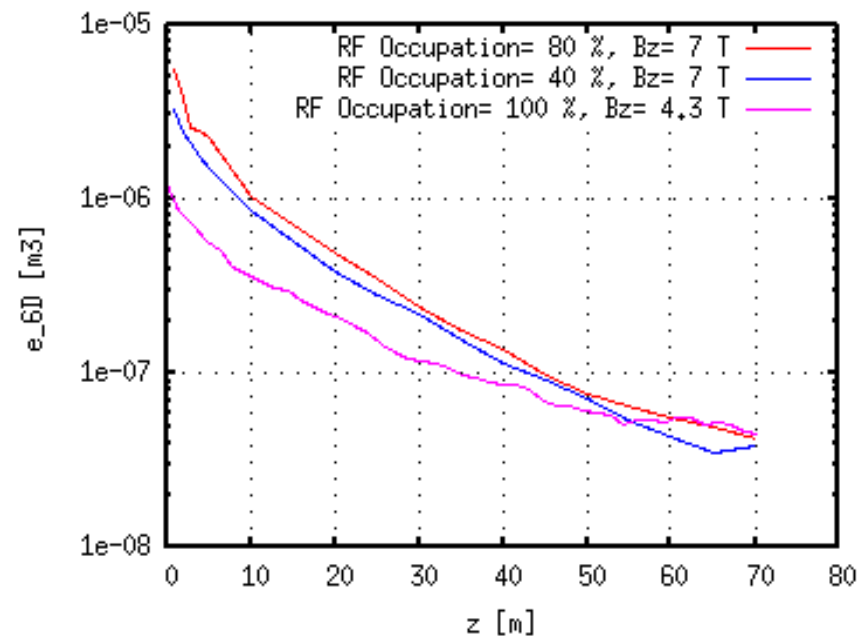

Figure 4.3.4: Cooling test in the type 2 model with various coil thickness. There are 20 coils per helix period.

\subsubsection{Practical Implementation}

There are some technological challenges to realize type 1 and type 2 models. The electrically separated RF cavities in the HCC must be excited individually. To achieve this, each cavity needs to be individually connected to the RF power inlet, or alternatively a coupling type cavity could be used. In either case, a gap between the cavity and the coil is needed to mount a wave guide. In addition, extra space will be required for thermal isolation between the cavity and the coil to avoid transferring the resistive heat on the cavity surface to the superconducting helical solenoid coils. A mechanical support structure for the coil and the cavity is also needed.

In the type 1 model, the cavity size is restricted in the radial direction. Adding a further gap between the coil and the cavity (e.g. for a waveguide or coupler) would reduce the acceptance further due to the transverse electric field variation in the pillbox cavity. In the type 2 model, the realistic minimum length of the RF cell needs to be understood. The impact of the longitudinal gaps between solenoids on the magnetic field quality seems 
not to be crucial. An advantage of the type 2 model is that the RF power can be connected to each cavity outside the helical solenoid coils.

\section{6D Cooling Experiment}

An important part of the future MCTF program is to perform an experimental test of the HCC theory, technology, and simulations. We are considering making a first test without $\mathrm{RF}$, using a magnet with tapered fields that follow the muon momentum decrease in the absorber. In addition to the simplification of avoiding RF cavities, this would allow liquid Helium (LHe) to be used as absorber instead of high pressure gaseous Hydrogen, which alleviates some safety concerns. This initial experiment has been called MANX, for Muon Collider and Neutrino Factory Experiment, although it is not required for a Neutrino Factory. The primary goals of the experiment would be several, including

- To demonstrate 6D cooling, in particular in the longitudinal direction since ionization cooling is inherently transverse in nature, and the MICE experiment will demonstrate transverse cooling.

- To verify the simulation codes to the level that the long channels needed for a collider can be simulated with confidence.
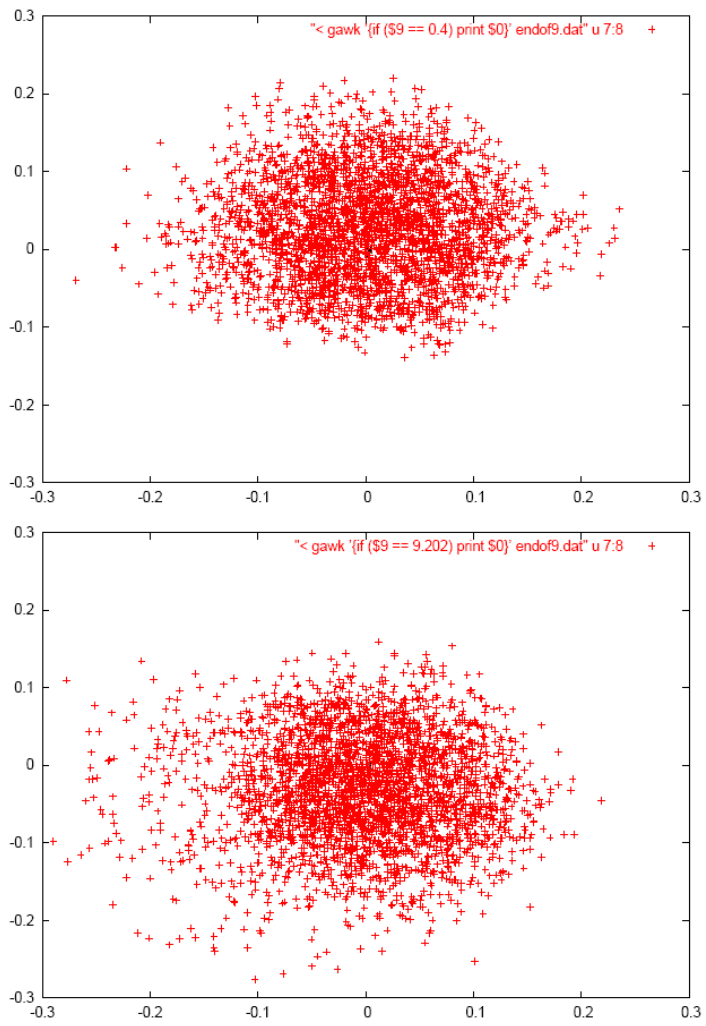

Figure 5.0.1: Particle distributions going into and out of a momentum dependent HCC (with matching sections). 
With the momentum dependent HCC, the cooling would have to be observed in terms of normalized emittance. The physical emittance may actually grow slightly as the beam looses momentum (see Figure 5.0.1). A cooling demonstration with a momentum dependent HCC would be the first step in demonstrating the practical usefulness of HCCs. If MANX is chosen as the first 6D cooling experiment, it is foreseen that an experiment with RF would follow.

\subsection{Experimental concepts}

There are two beam concepts for the experiment. One is following the MICE idea of detecting single particles entering and exiting the cooling channel. The advantage of this method is that a full set of 6D phase space coordinates would be measured for each muon "event". This allows virtually any analysis to be done in post-processing, once enough data has been obtained. Although in principle events can be weighted to account for an uneven initial distribution of events, it is important to make sure that sufficient data is collected for the entire initial phase space of interest (i.e. no 'dead spots'), thus the need for a properly designed matching section. Still to be understood is the required single particle measurement accuracy as well as the number of events needed to make an adequate measurement. According to studies done by the MICE collaboration, a few thousand events is sufficient to demonstrate cooling. However, it is likely that a significantly larger data set will be needed to verify simulations to the required accuracy. Simulations are underway to understand these issues better. In particular Muons, Inc is developing a code called G4MANX (based on the G4MICE code of the MICE collaboration), to allow simulations with realistic detector model.

An alternative beam concept, called the 'beamlet' or 'macroparticle' method is based on actively scanning a small pencil beam across the aperture of the HCC and recording the average coordinates of the beam as it enters and exits the structure. This approach is based on the fact that it is the average energy loss in the absorber that produces cooling, whereas stochastic effects such as energy straggling and multiple scattering, produce heating. Therefore, a mapping of the average behavior of an ensemble of particles with approximately the same initial parameters yields information about the cooling properties of the device. A separate measure of the heating properties can be obtained by measuring the emittance increase of the pencil beam, as it passes through the cooling device. The main advantage with the beamlet method is that simpler (and therefore cheaper) detectors could be used as long as the beam intensity is sufficiently high ( 1000 particles or more). A drawback is that it is difficult to measure the time coordinate. The variation in beam transit time across the aperture is of the order of a few nanoseconds, which is much shorter than any beam that could reasonable be produced. Therefore, the time coordinate would most likely have to be measured using single particle TOF (time-of-flight), which would probably require a lower intensity. In addition, the measurement of beamlet emittance (to get the heating term) appears to be best done using the single particle method. Therefore, once could envision a staged experiment where first the 5D cooling properties are measured using a beamlet and simple integrating electronics, and then the time dispersion and heating properties are measured with upgraded electronics capable of measuring single particles. This would have the advantage of providing cooling results fast, while at the end still generating the full 6D single particle data set for more detailed analysis. The actual detectors could be the same in both cases, for example, scintillating fiber tracking detectors. 
Another advantage with the beamlet method is that the active scanning ensures that the entire aperture can be covered, thereby possibly eliminating the need for matching sections (there are other reasons why one may want to match the beam into a solenoid field). By moving the detectors along with the beam position, the number of channels could also be significantly reduced with respect to the single particle experiment.

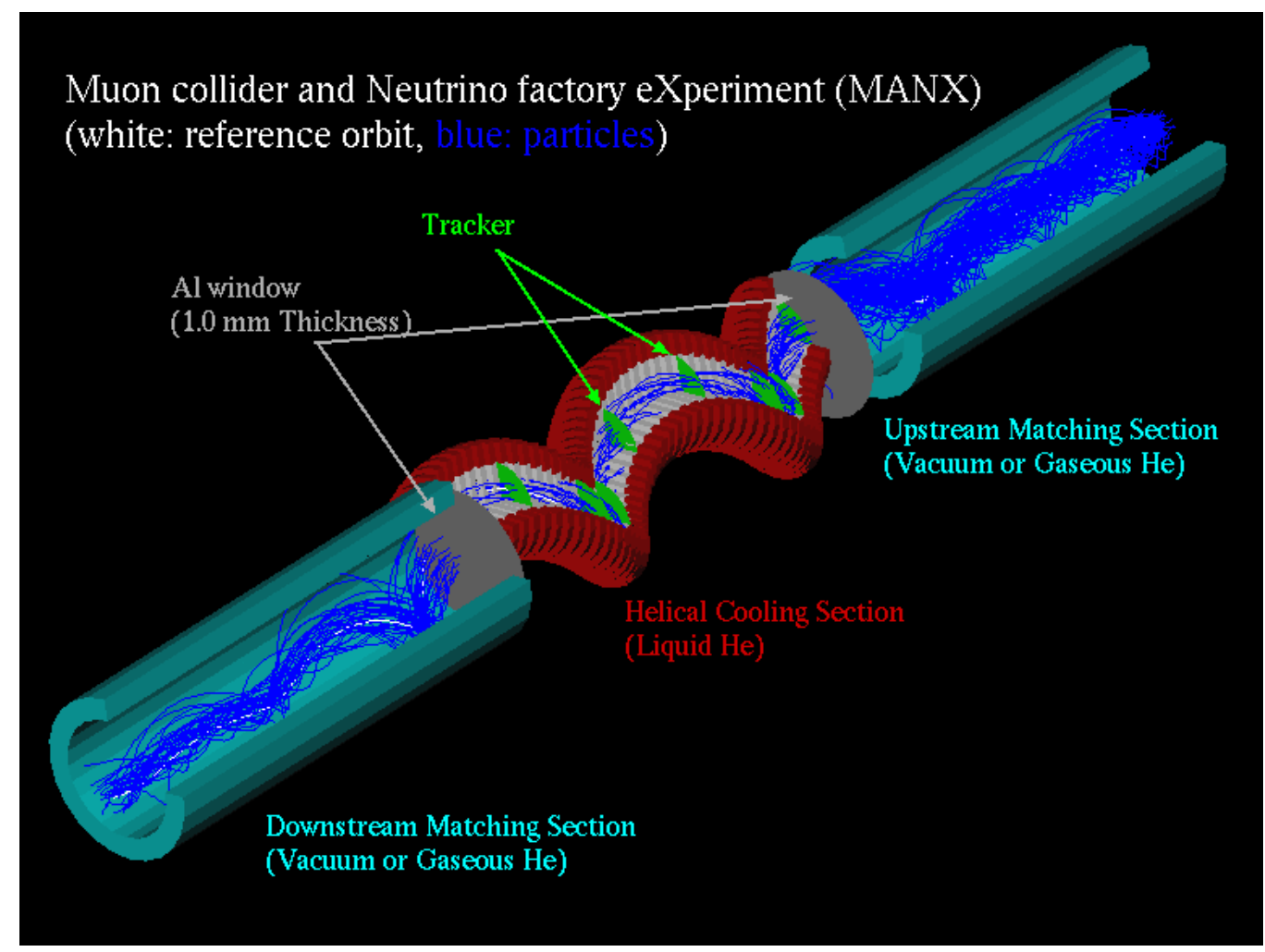

Figure 5.2.1: Conceptual layout of MANX

Table 5.2.1: Design parameters in cooling section

\begin{tabular}{|c|c|c|}
\hline $\begin{array}{l}\text { Initial mean momentum } \\
\text { Final mean momentum }\end{array}$ & $\mathbf{P}$ & $\begin{array}{l}300 \mathrm{MeV} / \mathrm{c} \\
170 \mathrm{MeV} / \mathrm{c}\end{array}$ \\
\hline Helical pitch & $\kappa$ & 1 \\
\hline Helical period & $\lambda$ & $1.6 \mathrm{~m}$ \\
\hline Helical ref. orbit radius & $A$ & $0.255 \mathrm{~m}$ \\
\hline $\begin{array}{l}\text { Initial solenoid strength } \\
\text { Final solenoid strength }\end{array}$ & B & $\begin{array}{l}-3.8 \mathrm{~T} \\
-1.7 \mathrm{~T} \\
\end{array}$ \\
\hline $\begin{array}{l}\text { Initial helical dipole strength } \\
\text { Final helical dipole strength }\end{array}$ & B & $\begin{array}{l}1.2 \mathrm{~T} \\
0.8 \mathrm{~T}\end{array}$ \\
\hline $\begin{array}{l}\text { Initial helical quad. strength } \\
\text { Final helical quad. Strength }\end{array}$ & $\mathbf{b}^{\prime}$ & $\begin{array}{l}-0.9 \mathrm{~T} / \mathrm{m} \\
-0.5 \mathrm{~T} / \mathrm{m}\end{array}$ \\
\hline
\end{tabular}

\subsection{MANX magnet}

The current MANX channel design consists of the three sections (Fig. 5.2.1); the upstream matching section, the helical cooling channel (HCC), and the downstream 
matching section. The HCC section is made of a superconducting helical solenoid coil (red rings in Figure 5.2.1). The higher order field components, for example the sextupole components, are not considered in the current design since they do not have a large influence on the cooling performance of a channel that has no energy compensating RF cavity. Figure 5.2.2 shows the field amplitude on the reference orbit. The field amplitude in the HCC magnet is reduced along the beam path as the particle loses kinetic energy in the liquid helium. The reduction of mean beam momentum as a function of distance in the absorber can be calculated using the Bethe-Bloch formula, and the field components in the cooling magnet can be computed using Equations (4.1) - (4.7).

The design parameters for the cooling section are listed in Table 5.2.1. The matching section field consists of a pure solenoid component plus a helical dipole field, that adiabatically ramps up from zero and generates the required beam transverse momentum and axial position offset. The length of the matching magnet is determined by the period of the longitudinal betatron oscillation $(\sim 0.75 \lambda)$ multiplied by an integer: length $=0.75 \lambda$ $\times \mathrm{n}$, where $\lambda=1.6$ meters and $\mathrm{n}=2$ is minimum. Hence the length of matching magnet is 2.4 meters in the current design. To stabilize the beam phase space, a helical quadrupole field component is added with the same ramping rate as the helical dipole field. The ramp-up of the helical field components induces a transverse instability. To stabilize the beam, the solenoid field component is initially higher than that in the helical solenoid by a factor which is typically $\sim 1.5$, and is found by iterating. The solenoid field is adiabatically ramped down to connect to the solenoid field component in the magnet. A similar concept is used for the design of the downstream matching magnet. The simulations use a matching field map that assumes ideal helical current conductors.

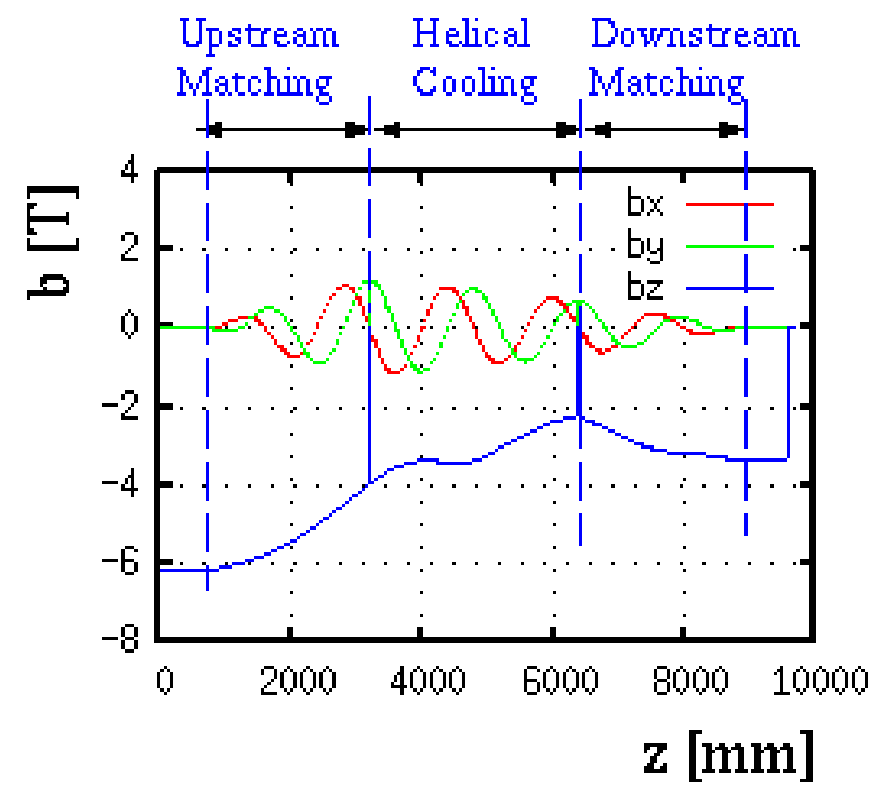

Figure 5.2.2: Magnetic field of reference particle in MANX

Liquid helium is used as the $\mathrm{dE} / \mathrm{dx}$ medium in the current design, avoiding the safety issues involved in handling large volumes of hydrogen. Liquid helium is also used as coolant for the superconducting coils. Since the muon beam emittance in the HCC remains well above the equilibrium emittance, the ionization cooling efficiency in liquid helium is similar to the corresponding efficiency for liquid hydrogen (the reduction is less 
than 10\%). The radiation length in liquid helium is longer than in liquid hydrogen, but at the same time the energy loss rate in liquid helium is smaller than that in liquid hydrogen.

To contain the liquid Helium, windows are required at both ends of the cooling channel. The current window is a $1.0 \mathrm{~mm}$ thick aluminum plate which is approximately 4 times thicker than the vacuum window in the MICE experiment. Simulations indicate that this window thickness should not be an issue because of the big cooling factor expected in the MANX helical solenoid. Window thicknesses up to $5.0 \mathrm{~mm}$ were tested in simulations without any significant cooling efficiency reduction. This result also encourages us to add a thermal isolation window which consists of a superinsulator layer and a liquid nitrogen shielding wall. It would be desirable to have detectors inside the cryostat to detect the particle positions and monitor the particle loss. It may be possible to used scintillating fibers for this purpose. Tests of scintillation fibers in cryogenic conditions are in progress.

\subsection{Simulations}

Figure 5.3.1 shows the simulated transverse and longitudinal emittance evolutions in MANX. The cooling factors in the transverse and longitudinal directions are similar, about $\sim 1.2$. In this simulation, the injected beam was smaller than the aperture and not perfectly matched. The cooling factor may be increased by injecting a larger and better matched beam at the cooling magnet. Unfortunately, the acceptance is limited by the matching magnet. The current matching magnet also generates heating in transverse and longitudinal phase space as shown in Figure 5.3.1. This problem could perhaps be solved by introducing higher order field component in the matching magnet to compensate for spherical aberration. Making the matching magnet longer to reduce the ramping rate of the helical dipole field component would also help. Figure 5.3.2 shows the 6D emittance evolution. The current $6 \mathrm{D}$ cooling factor in the cooling magnet is 2.0 , but if the blow-up in the matching sections is taken into account, the overall cooling effect is less.
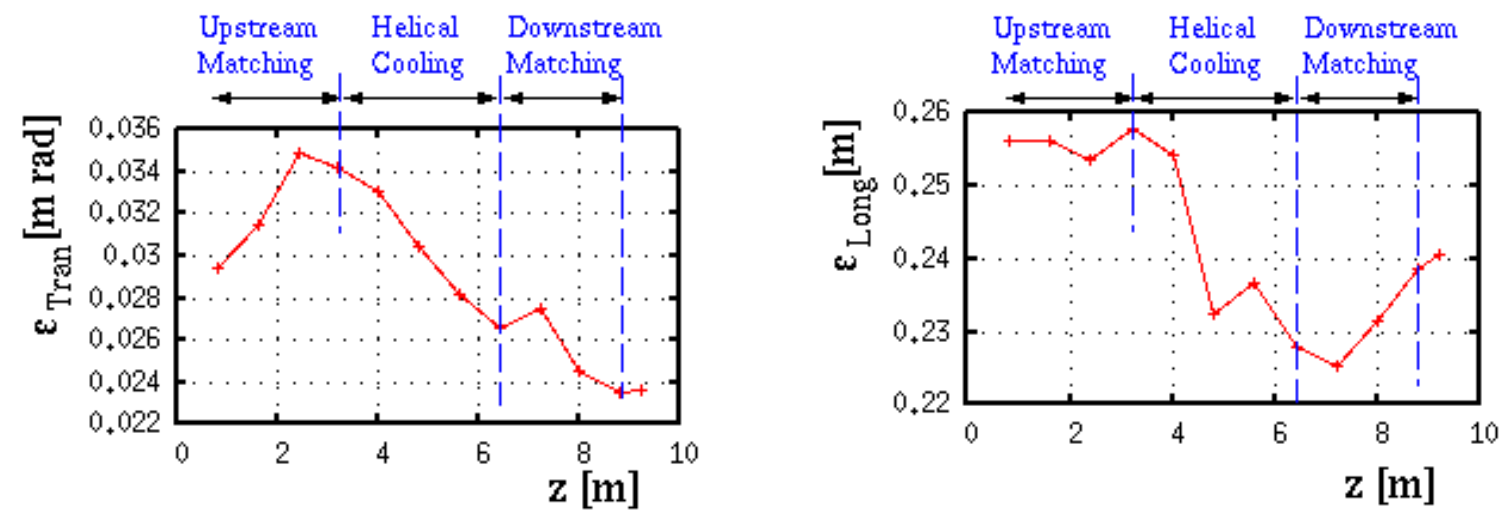

Figure 5.3.1: Transverse (left) and longitudinal (right) emittance evolutions in MANX 


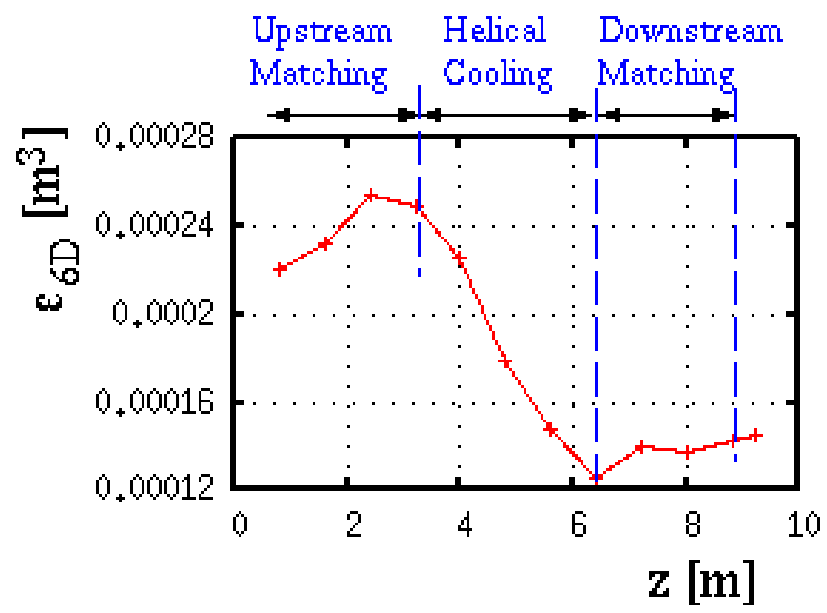

Figure 5.3.2: $6 D$ emittance evolution in MANX

\subsection{Tuning the cooling partitions}

The cooling decrements in transverse and longitudinal phase spaces can be tuned by changing the dispersion function, $\hat{D}$. For instance, the channel can be tuned for longitudinal cooling only at $q=0$, hence $\hat{D}=21+\kappa^{2} / \kappa^{2}$ from eq. (4.4). In this condition, the helical dipole field component must be zero and a stronger helical quadrupole field component is needed to stabilize the beam phase space. Figure 5.4.1 shows the transverse and longitudinal emittance evolutions with two different cooling decrements, one is the longitudinal only cooling decrement and other is the equal cooling decrement, respectively. A test channel in this study consists of the upstream matching and the cooling sections. Figure 5.4.1 shows that the transverse cooling decrement in the equal cooling decrement channel is much bigger than that in the longitudinal only cooling channel. On the other hand, the longitudinal emittance reduction in the longitudinal only cooling channel has much bigger than that in the equal cooling decrement channel. This result shows that longitudinal only cooling can be made in simulation. It has yet to be determined if and how this can be done practically in the helical solenoid setup, as well as what tuning range is needed for the experiment. Figure 5.4.2 shows the $6 \mathrm{D}$ emittance evolution in the equal cooling decrement and longitudinal only cooling decrement channels. The slopes in both cooling decrements are same, which means that the $6 \mathrm{D}$ cooling efficiency is determined by the total amount of energy loss.

The matching magnet design for the equal cooling partition magnet does not work well for longitudinal cooling only, since the helical dipole field component should be zero in the cooling magnet. This makes a significant field jump between the matching and the cooling magnets. Figure 5.4.3 shows the field amplitude in the longitudinal only cooling channel including with the upstream matching channel. The field strength immediately goes down at the boundary between the matching to the cooling magnets $(\mathrm{z}=3.2 \mathrm{~m})$. Some correction coils could perhaps be used to remove the helical dipole field component in the cooling section. This study is in progress. 

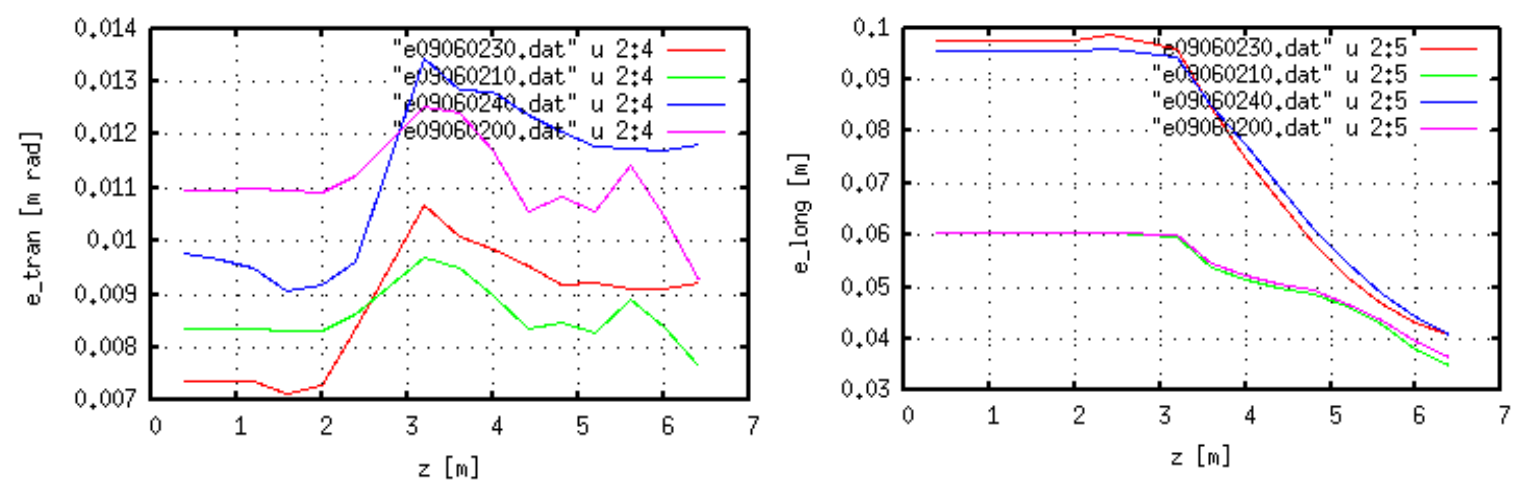

Figure 5.4.1: Transverse (left) and longitudinal (right) emittance evolutions in the equal cooling decrement and the longitudinal only cooling decrement channels. "e09060230.dat" and "e09060240.dat" are the emittance evolutions in the longitudinal only cooling decrement channel with the different initial beam phase spaces while "e09060210.dat" and "e090200.dat" are the emittance evolutions in the equal cooling decrement channel with the same initial condition as in "e09060230.dat" and "e09060240.dat", respectively.

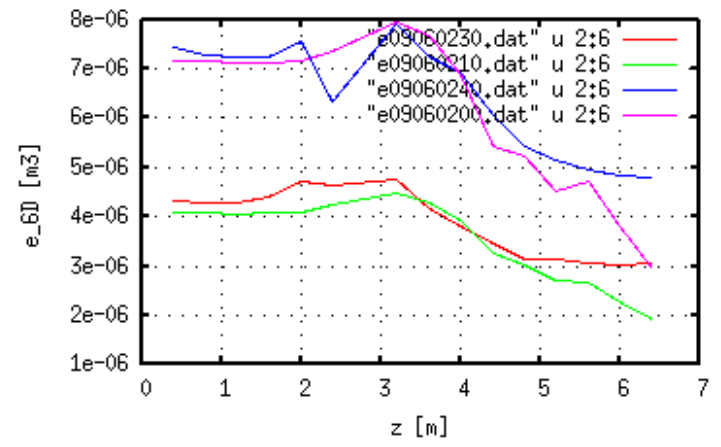

Figure 3.4.2: $6 D$ emittance evolutions in the equal cooling decrement and the longitudinal only cooling decrement channels.

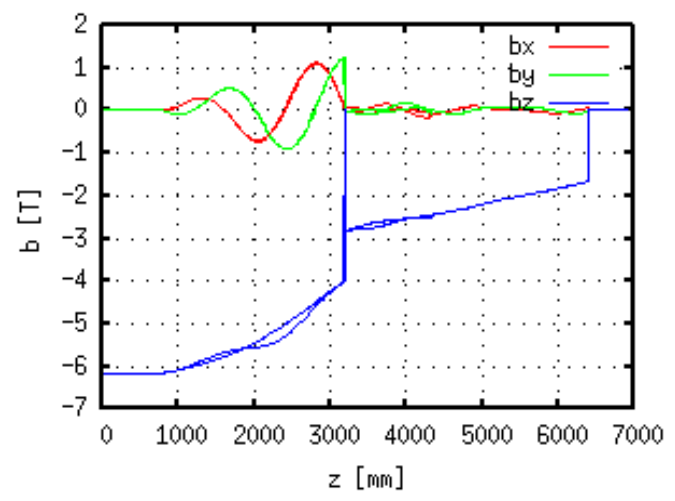

Figure 5.4.3: Field amplitude in the longitudinal only cooling decrement channel with the upstream matching magnet. The field is immediately turned off at $z=3200 \mathrm{~mm}$ 


\subsection{Field error sensitivity}

Figure 5.5.1 shows the transverse and longitudinal emittance evolution in the cooling magnet with various field errors. The simulated field error distribution is Gaussian with an rms of $\pm 2 \%, \pm 5 \%, \pm 7 \%$, and $\pm 10 \%$. These fluctuations are applied to three field components, bx, by, and bz. From simulation results, it is clear that the random field error does not affect on the cooling efficiency, and the longitudinal phase space is more sensitive to the field error than the transverse one. Note that the field errors in this study did not satisfy Maxwell's equations. The real field errors must be produced by misaligning the helical solenoid coils and the instability of currents. This study has yet to be done. At muon momenta below $150 \mathrm{MeV} / \mathrm{c}$ ( $\mathrm{z}>4$ in the figure) longitudinal heating it is usually observed.
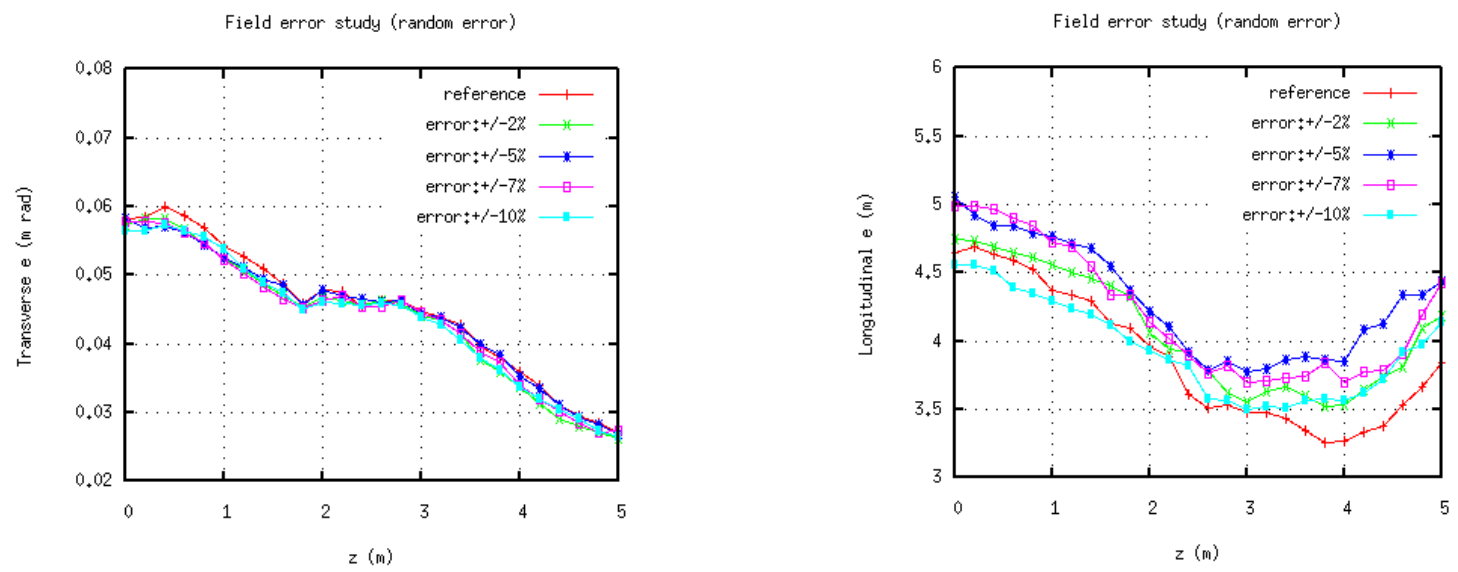

Figure 5.5.1: Transverse (left) and longitudinal (right) emittance evolutions in the cooling magnet with various field errors

\subsection{Muon beamline}

The possibility to make a muon beam for the $6 \mathrm{D}$ cooling experiment at the MTA facility has been studied. It would make use of a scheme that has been proposed to significantly increase the beam intensity available for MTA tests $\left[{ }^{27}\right]$. The scheme involves utilizing the front portion of the linac pulse that would otherwise have been sent to the momentum dump. Using two combined function sector magnets, the momentum dump beamline can be joined up with the MTA proton beamline (Figure 5.6.1). By also lengthening the linac pulse, around $2 \times 10^{14}$ protons/second could be available without using any dedicated $15 \mathrm{~Hz}$ linac cycles. Being decoupled from the Booster would be particularly useful, since protons are in ever increasing demand for the neutrino program. A preliminary muon beam design has been developed based on a carbon target immersed in a 5T solenoid, and a quadrupole decay channel $\left.{ }^{28}\right]$. It would be capable of providing $\sim 1 \times 10^{7}$ muons in the $250 \mathrm{MeV} / \mathrm{c}$ range per linac pulse. By collimating the beam to a dimension useful for a macro-particle experiment, about 10,000 muons per pulse could be achieved. This small pencil beam could be made very clean through the use of an electrostatic separator, several of which are available at BNL. Studies of this beamline are continuing. 


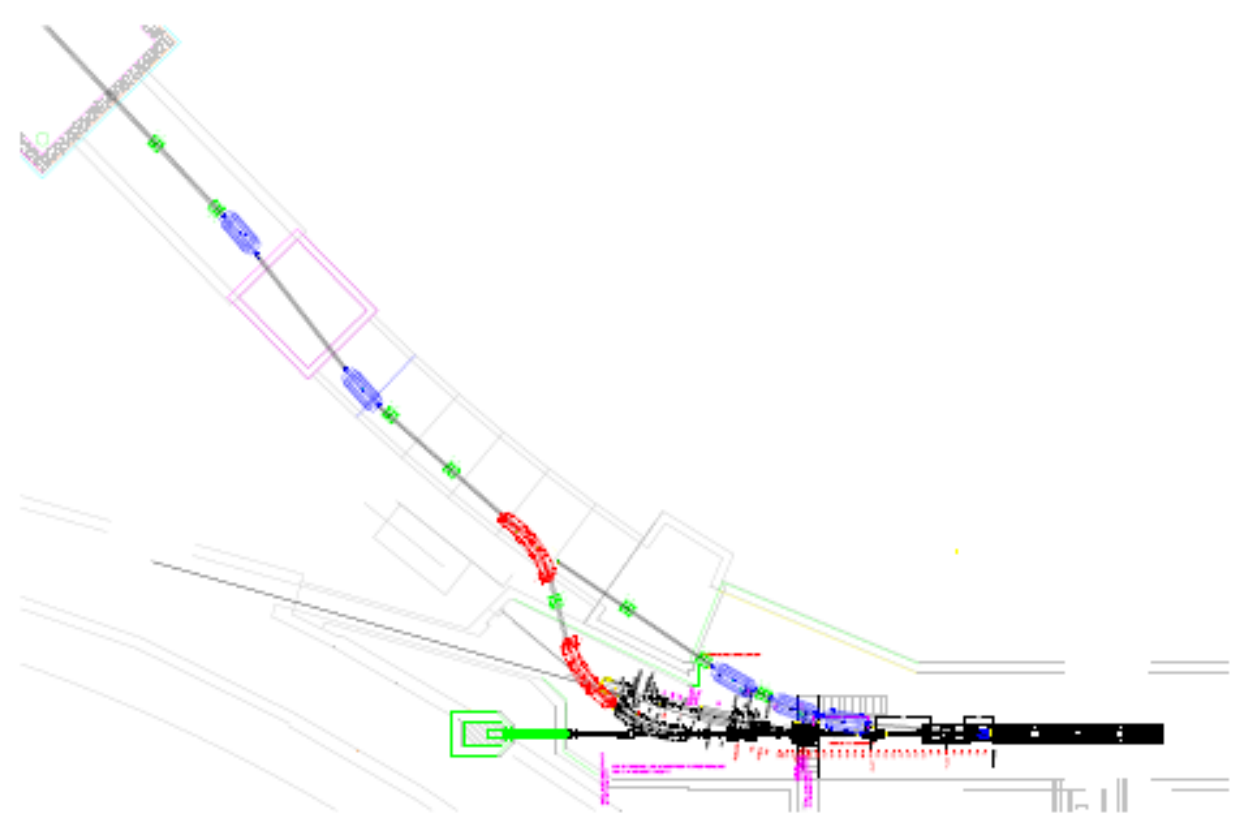

Figure 5.6.1. Proposed scheme to increase proton intensity to MTA. The blue components are part of the proton beamline. The red components would be added to utilize the beam from the momentum dump.

In addition to this, the possibility of using the $\mathrm{KTeV}$ hall for the experiment is being studied (see Figure 5.6.2). The hall is being considered for use by the E906 experiment, but it appears possible for the two experiments to share the area. Initial estimates indicate that up to $3 \times 10^{7}$ muons may be available per Main Injector spill. More detailed simulations are underway to verify this number and understand the beam properties.

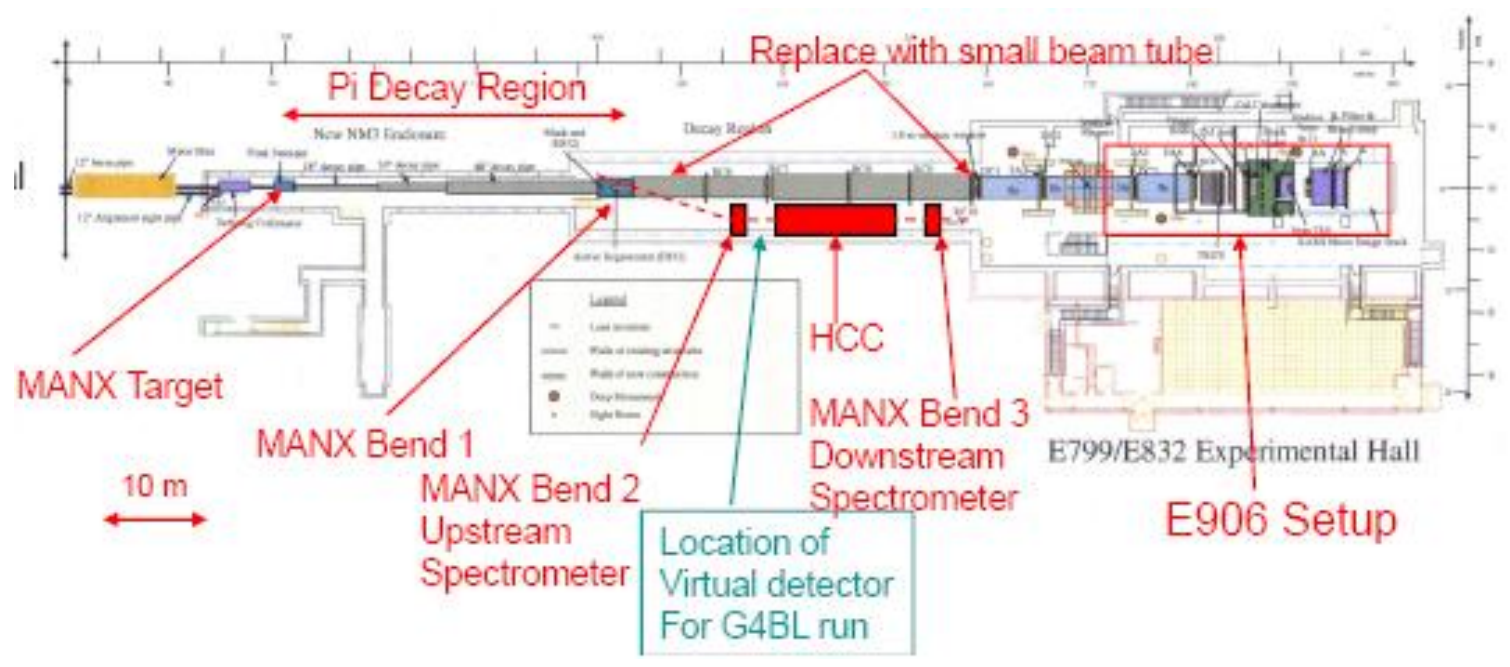

Figure 5.6.2. Schematic of possible layout for $6 \mathrm{D}$ cooling experiment (MANX) in $\mathrm{KTeV}$ hall.

Finally, the mu2e collaboration has recently submitted a letter of intent to propose a muon conversion experiment. This would make use of the Debuncher as a proton storage ring for slow spills after the Run II collider program is over. While this may not be timely 
for the initial $6 \mathrm{D}$ cooling experiment, it may present an interesting option for a follow-up experiment.

\section{High Field Solenoids}

The final Muon Collider cooling channel stages may require DC solenoid magnets with magnetic fields of 40-50 $\mathrm{T}$ in an aperture of $\sim 50 \mathrm{~mm}$. A $45 \mathrm{~T}$ hybrid solenoid with superconducting outer coils and copper inner coils has been successfully built and operated at NHMFL, however, the $\sim 30 \mathrm{MW}$ power consumption at the maximum field makes this magnet design impractical for accelerator applications. Other possible solutions for creating steady state solenoid fields of $\sim 50 \mathrm{~T}$ utilizing HTS conductor are being investigated, and different approaches for the coil structural design are being studied $\left[{ }^{29},{ }^{30}\right]$.

In addition, during the past 12 months studies have been performed on two kinds of HTS materials $\left[{ }^{31}\right]$ :

- $\quad \mathrm{Bi} 2 \mathrm{Sr} 2 \mathrm{CaCu} 2 \mathrm{O} 8-\mathrm{x}$ (BSCCO-2212) as a round multifilamentary wire, which can be cabled in the form of Rutherford-type cables.

- $\quad$ Anisotropic conductors, including (Bi,Pb)2Sr2Ca2Cu3Ox (BSCCO-2223) and second generation coated conductors (YBCO).

For the BSCCO-2212, tests have been performed to study the effects of $\mathrm{J}_{\mathrm{c}}$ from billet preparation and cabling parameters, primarily at $4.2 \mathrm{~K}$, along with SEM/EDS analysis. For the anisotropic conductors studies were performed on the angular dependence of the $\mathrm{J}_{\mathrm{c}}$ using a newly designed probe. All studies were performed in the Superconductor R\&D laboratory in the Fermilab Technical Division magnet systems department.

\subsection{Existing high field solenoids}

The viability of HTS materials in high field solenoids has been shown in two tests at the NHFML in Tallahassee. HTS inserts were successfully used in hybrid magnets to produce fields of $25 \mathrm{~T}$ and above. In 2003 , a $41 \mathrm{~mm}, 5.11 \mathrm{~T} \mathrm{Bi} 2212$ insert was tested in a

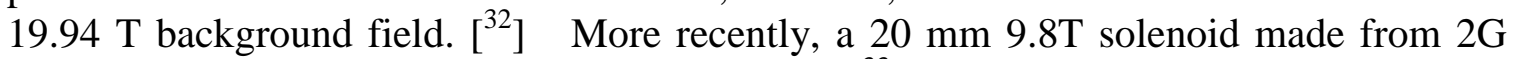
YBCO tape with tested in a $19 \mathrm{~T}$ background field $\left[{ }^{33}\right]$.

In both cases the background fields were generated from existing resistive solenoids.

\subsection{High field solenoids design}

Many of the HTS insert coils built up to date used either Bi-2223 or Bi-2212 tapes. Both materials have relatively well defined electromechanical properties and are available in sufficiently long piece-lengths. The studies were primarily focused on Bi-2223 tapes that are available with stainless steel reinforcements from American Superconductor. These tapes have field anisotropy. Also studied are Bi-2212 round wires from OST that have higher engineering current density which is isotropic, but are not reinforced. Recent 
advances in the YBCO conductor development have also made it a viable candidate for a high field solenoid.

To access the anisotropy of Bi-2223 tape, short sample measurements were performed as a function of field and orientation at the Fermilab Short Sample Test Facility. These field and angle dependences were parameterized using analytical functions, and extrapolated to higher fields using data on Bi-2212 wire, measured up to $45 \mathrm{~T}$ at NFMFL[ $\left.{ }^{34}\right]$. The "high strength plus" tape from American superconductor was selected for the solenoid design because of combined high engineering current density and good mechanical properties.

The round Bi-2212 wire has $\sim 30 \%$ higher engineering current density than the Bi-2223 "high strength plus" tape has in a parallel field. This could provide $\sim 15-20 \%$ higher average current density with respect to the tape if the round wires are composed into a Rutherford type cable. However, the relatively large cabling degradation measured at Fermilab in Bi-2212 cables advises against this option. Thus, it was assumed that the coils are wound from the round wire. The compaction factor of a coil made from round wire depends on the winding pattern and insulation material. Since the preferred insulation type is not known at the moment, it was conservatively assumed that the insulation is rigid enough so the wire does not follow the "grooves" of preceding layer. In this case, the theoretical compaction factor (not accounting for the insulation) is $\pi / 4=0.78$. Also, the round wires may likely require extra coating in addition to the interlayer insulation that would reduce the compaction factor down to $\sim 0.7$. This would bring the average current density down to essentially the same level as in Bi-2223 tape winding. So, the current density in Bi-2212 winding is chosen the same as in Bi-2223 tape in the parallel field.

A $~ 50 \mathrm{~T}$ solenoid would not significantly benefit from a ferromagnetic yoke that, if present, would mostly act as a fringe field screen. We can therefore analytically calculate the magnetic field in the absence of iron. A parametric analytical model was created for that purpose. The solenoid cross-section was subdivided to a number of turns and the total field was calculated as the superposition of all turn fields. The number of subdivisions was adjusted in order to achieve the desired calculation accuracy. It was determined that for our particular problem this approach provides faster convergence than a general solution of the finite solenoid field at the same calculation accuracy.

To maximize the coil efficiency it was assumed that each coil section operates at its maximum current density determined by the peak field in that section. In practice this can be achieved by adjusting the wire dimensions so that all sections operate at the same current or powering each section from an individual power supply. The former option requires only one pair or current leads and one power supply, while the latter option may be preferred for the quench protection. 


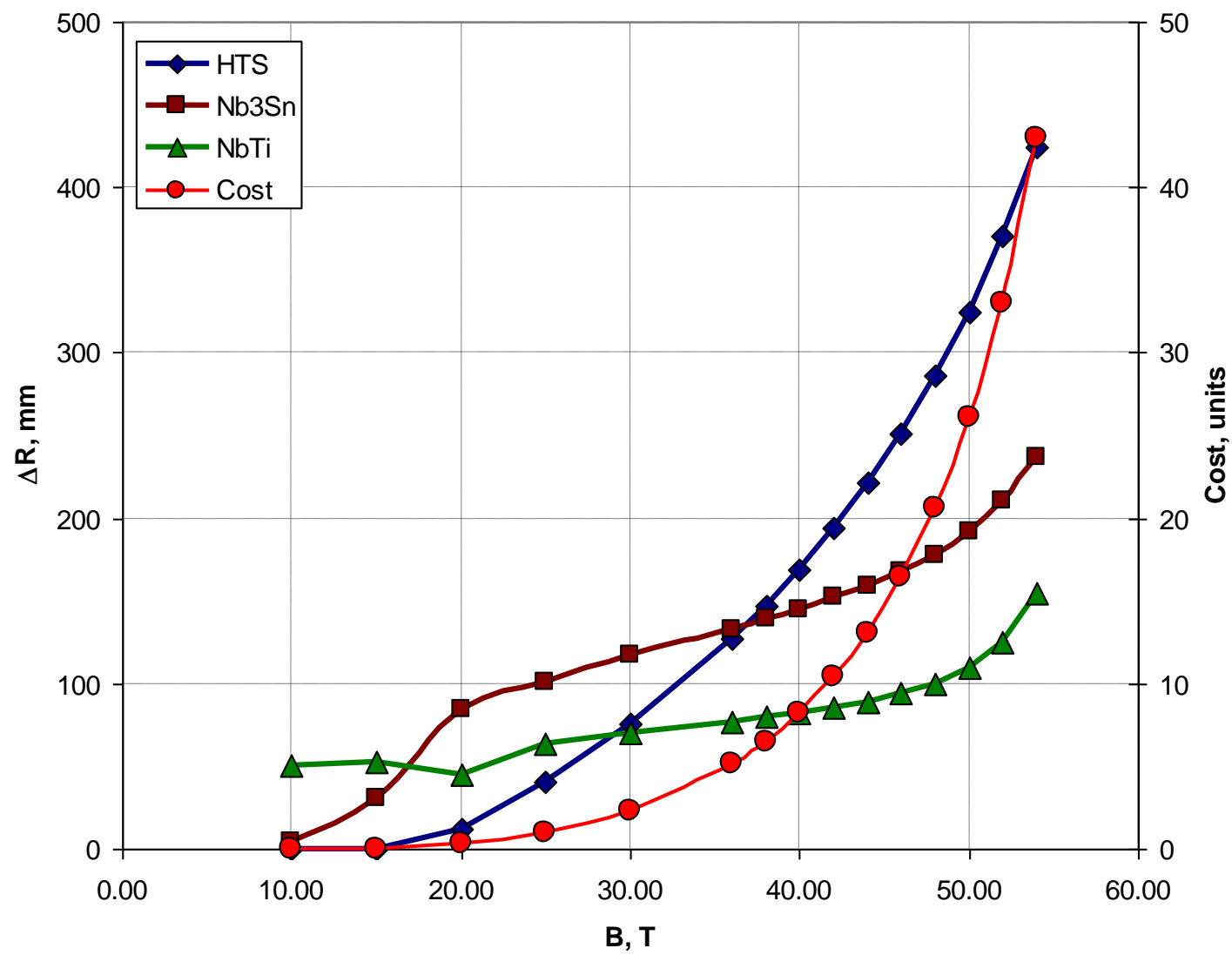

Figure 6.2.1. Coil radial thicknesses and relative superconductor cost as functions of magnetic field optimized for the minimum cost.

In order to find the optimum coil configurations, the relative cost factors were introduced. An objective function representing the sum of superconductor costs from each section was used during the coil optimization. Figure 6.2.1 shows an example of the radial thicknesses of HTS, $\mathrm{Nb}_{3} \mathrm{Sn}$ and $\mathrm{NbTi}$ sections and superconductor cost as functions of the magnetic field, optimized for the minimum cost at each field level. Each section was powered from a separate power supply and had $25 \%$ of structural material in the crosssection. Figure 6.2.2 shows a similar plot, except the optimization criterion was the minimum magnet outer diameter. 


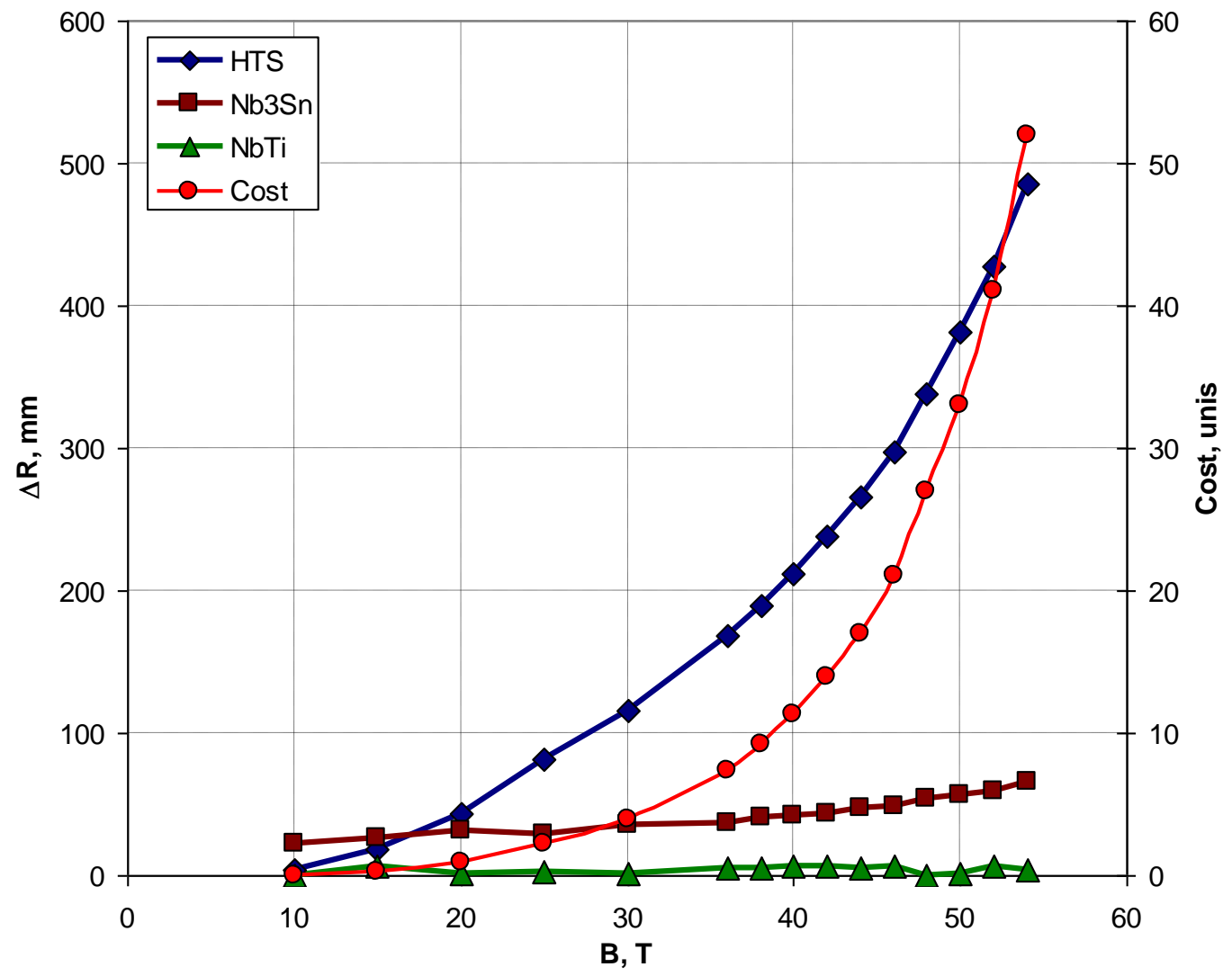

Figure 6.2.2. Coil radial thicknesses and relative superconductor cost as functions of magnetic field optimized for the minimum size.

After the optimum coil dimensions were determined, a numerical structural analysis was performed. In order to limit the mechanical stresses in the coil at high field, the HTS section was subdivided into several mechanically decoupled sub-sections, each surrounded by the inner and outer shells. The pre-stress was provided by pretension of the outer shells. Figure 6.2.3 shows an example of the azimuthal stress distribution in the HTS section at zero field and at $50 \mathrm{~T}$. The stresses are within +/- $160 \mathrm{MPa}$, however because of the added structural material the maximum field is only $44 \mathrm{~T}$.

The next steps will involve iterating the structural analysis with analytical optimization in order to find an acceptable solution for the field, stresses and cost. The magnet fabrication technology will also need to be defined. 

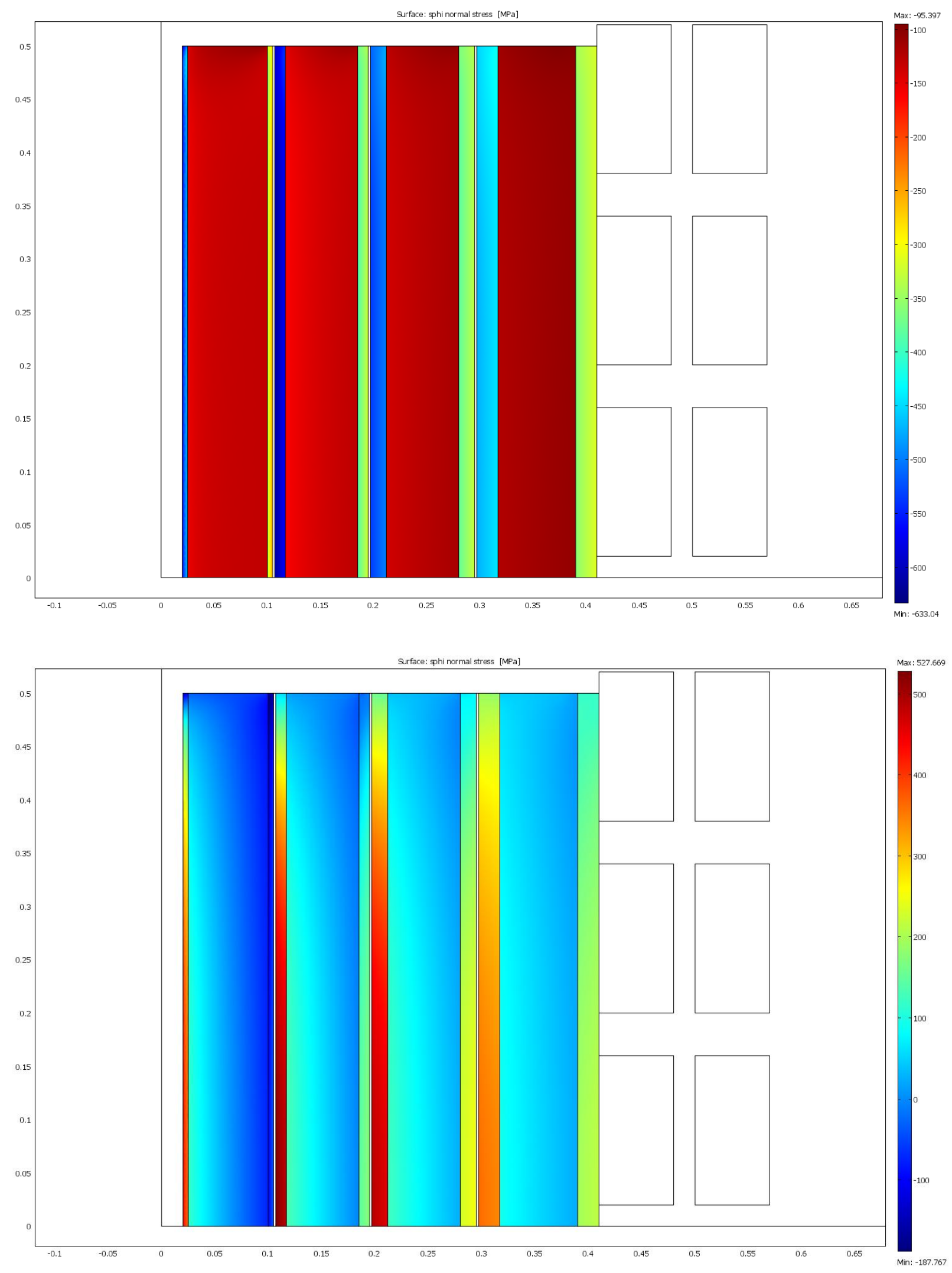

Figure 6.2.3. Azimuthal stress distribution in the HTS coil at zero field (top) and at $50 \mathrm{~T}$ field (bottom). 


\subsection{Other Design Considerations for High Field Solenoids}

- HTS superconductors may be limited to a stress of $0.4 \%$. The amount of stainless steel needed to contain hoop stress, which increases linearly with radius, is large and causes the size and energy stored in a solenoid to grow. A metal ductile at $4 \mathrm{~K}$ that is stiffer than stainless steel would take up less volume. Rhenium meets this requirement but annual production is only 50 tons, mostly from Arizona, Chile, and Kazakhstan. Tungsten and molybdenum are both stiffer than iron. All three have body centered cubic lattices which are brittle at cryogenic temperatures. Iron can be made ductile by adding nickel. Finding similar alloying agents for tungsten or molybdenum could lead to smaller magnets.

- Often superconducting magnets are designed to deposit the stored magnetic field energy into the coils when the magnet quenches. This can lead to larger magnets and more stored field energy. External energy dumps may be worth investigation. The 5.6 MJ superconducting solenoid at the Fermilab D0 experiment is designed to optionally use an external $277 \mathrm{~kg}$ stainless steel block as an energy dump.

- The $\$ 15$ million 45T solenoid at NHMFL in Florida uses $30 \mathrm{MW}$ to run a 30T copper coil inside a 15T superconducting coil. What would happen if the situation could be reversed? A $15 \mathrm{~T}$ copper coil inside a 30T superconducting coil would only consume 8 MW. A series of such magnets might be a viable option for muon cooling, if high field pure superconducting solenoids are delayed.

\subsection{BSCCO-2212 wire and cable studies}

$\mathrm{Bi}_{2} \mathrm{Sr}_{2} \mathrm{CaCu}_{2} \mathrm{O}_{8-\mathrm{x}}$ (BSCCO-2212) is one of a large number of copper-oxide High Temperature Superconductors (HTS), which in addition to much higher critical temperatures also have very high critical fields compared with Low Temperature Superconductors (LTS). However BSCCO-2212 is the only copper oxide material which can be easily melt processed, which enables it to be fabricated in a wide variety of shapes, including conventional round multifilamentary wire.

For our studies BSCCO-2212 billets were produced by OST. The best performance is usually obtained by oxygen preannealing of the $\mathrm{AgMg}$ sheathed strand. However, the hardened sheath which results from this preanneal severely restricts the diameter around which the wire can be bent without cracking. Out of the several billets produced, three annealing processes where studied in detail (noted as A,B, D in the figures). Wires were drawn to diameters ranging from 0.7 to $1.02 \mathrm{~mm}$. From the wire produced, we fabricated a number of Rutherford-type cables with packing factors ranging from $81 \%$ to $86 \%$; typical for Rutherford cables in accelerator magnets. Samples were heat treated at OST according to an optimized schedule. The transport performance at $4.2 \mathrm{~K}$ of round and extracted strands was measured up to $15 \mathrm{~T}$, and a number of cables were tested at selffield. SEM/EDS analysis were also performed. In the case of round strands (not made into cable), not much difference in $J_{\mathfrak{c}}$ was found between the two different annealing processes used. The plot in Figure 6.4.1 of the normalized $\mathrm{I}_{\mathrm{c}}(4.2 \mathrm{~K})$ and that in Figure 6.4.2 of the normalized $\mathrm{n}$-value, both as a function of cable packing factor, summarize the results obtained for all the extracted $\mathrm{Bi}-2212$ strands. There is no noticeable dependence on magnetic field for either $I_{c}$ or $n$-value. As can be seen, besides for one single case, which however was found to be reproducible, the $I_{c}$ degradation of the extracted strands 
is reasonable and typically less than $20 \%$ at least up to $85 \%$ packing factor. The n-value dependence with packing factor is stronger than for the $I_{c}$. Strands of different designs behave differently to cabling. For instance the $I_{c}$ degradation is larger for strand $B$, which is an old design that had not been optimized for cabling.

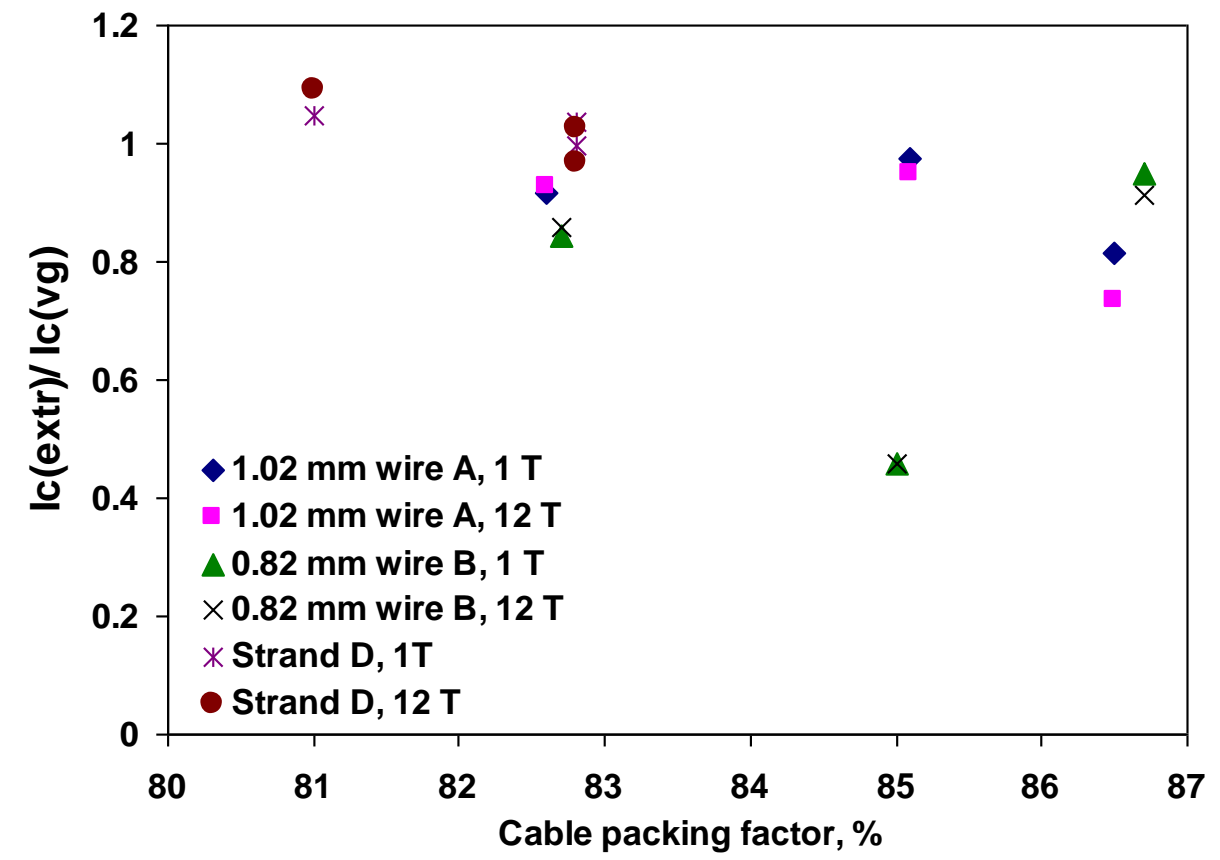

Figure 6.4.1. Normalized $I_{c}$ at $4.2 \mathrm{~K}$ as a function of cable packing factor for the extracted Bi-2212 strands.

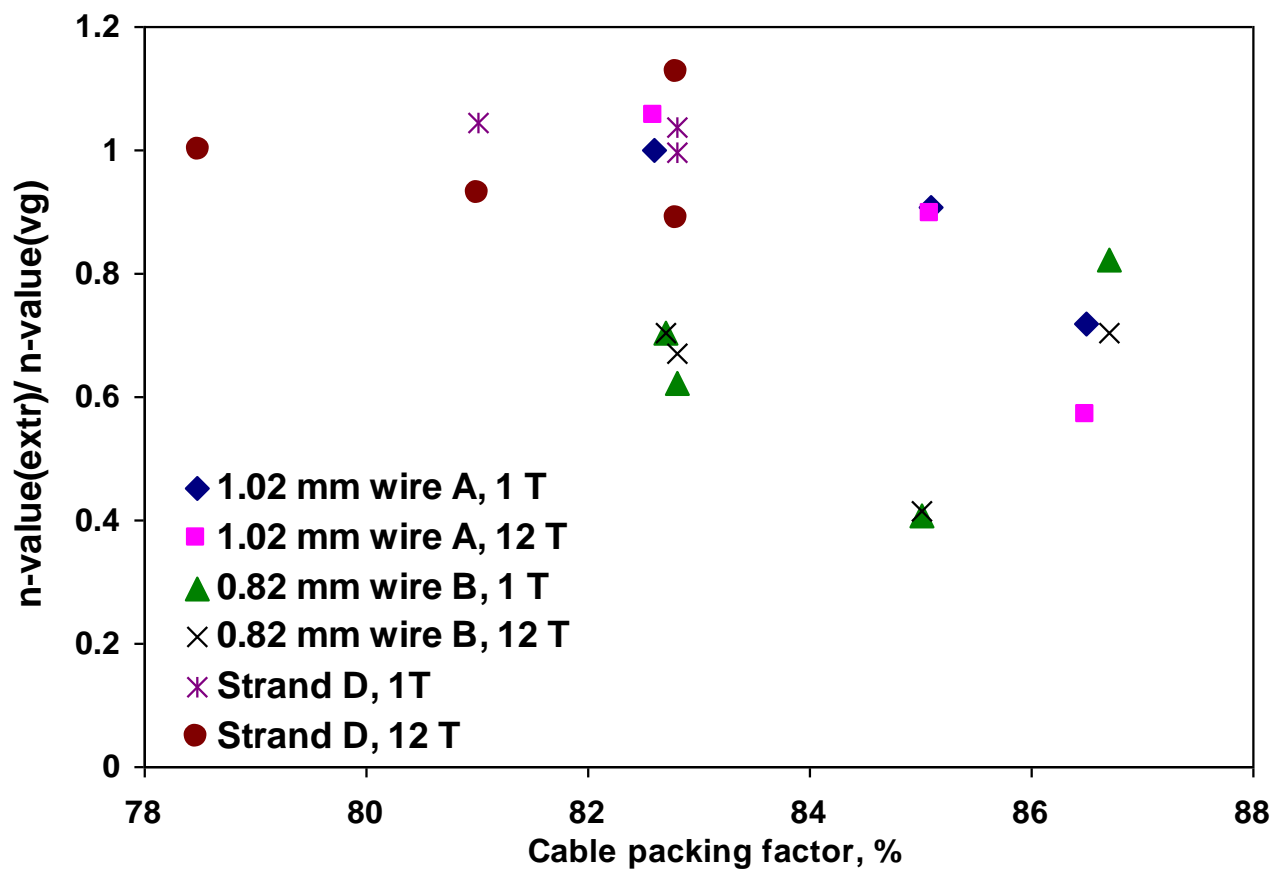

Figure 6.4.2. Normalized n-value at $4.2 \mathrm{~K}$ as a function of cable packing factor for the extracted Bi-2212 strands. 
To better understand the behavior of the extracted strand with larger degradation, SEM/EDS analysis was performed on the surface of tested samples with larger and smaller degradation. It was found that both samples had $\mathrm{MgO}$ precipitates on the surface of the silver sheath consistent with the original $\mathrm{Ag} 0.2 \% \mathrm{Mg}$ alloy composition. However, the distribution density of the fine $\mathrm{MgO}$ precipitates in the "large degradation cable" was larger than that in the "smaller degradation cable. In addition, many abnormally large $\mathrm{MgO}$ precipitates were found in the large degradation strand, whereas small degradation strand did not have any. These large precipitates may be caused by irregular grain growth, possibly due to heat treatment temperature inhomogeneities, but additional SEM studies of the cross section of the extracted strands should be performed to understand whether this effect is reproducible.

The cables were measured at self-field with a SC transformer equipped with a Rogowski coil to measure the secondary current. For all the cable, an $\mathrm{I}_{\mathrm{c}}$ degradation of about $50 \%$ was found. This current reduction on the cables was significantly and systematically larger than that of the extracted strands. To estimate the expected cable current, the average of the extracted strand critical currents at $0 \mathrm{~T}$ and $0.5 \mathrm{~T}$ was used, along with the cable strand composition, because the self-field produced by the transformer secondary is $0.18 \mathrm{~T}$ to $0.29 \mathrm{~T}$ for cable currents between $3000 \mathrm{~A}$ and $5000 \mathrm{~A}$. More accurate estimates could be made in the future.

The $I_{c}$ degradation measured on the cable samples was significantly and systematically larger than that of the extracted strands. From SEM analysis it was found that this was due to changes in the microstructure and chemical composition due to powder leaks during heat treatment. However, because no leaks were observed on the extracted strands, which performed well, this problem may not be as much related to the strand ability to withstand deformation as to the heat treatment of the cables itself. Given the excellent performance of the $\mathrm{Bi}-2212$ strands after cabling, it is worthwhile to devote future resources to solve this cable heat treatment problem.

\subsection{Angular measurements of HTS critical current}

A new sample holder was built to measure the $\mathrm{I}_{\mathrm{c}}$ angular dependence. The probe as used to measure the field dependence on BSSC-2223 and YBCO tape. A BSCCO-2223 Hermetic tape and a 2G 348 Coated conductor produced by AMSC were tested.
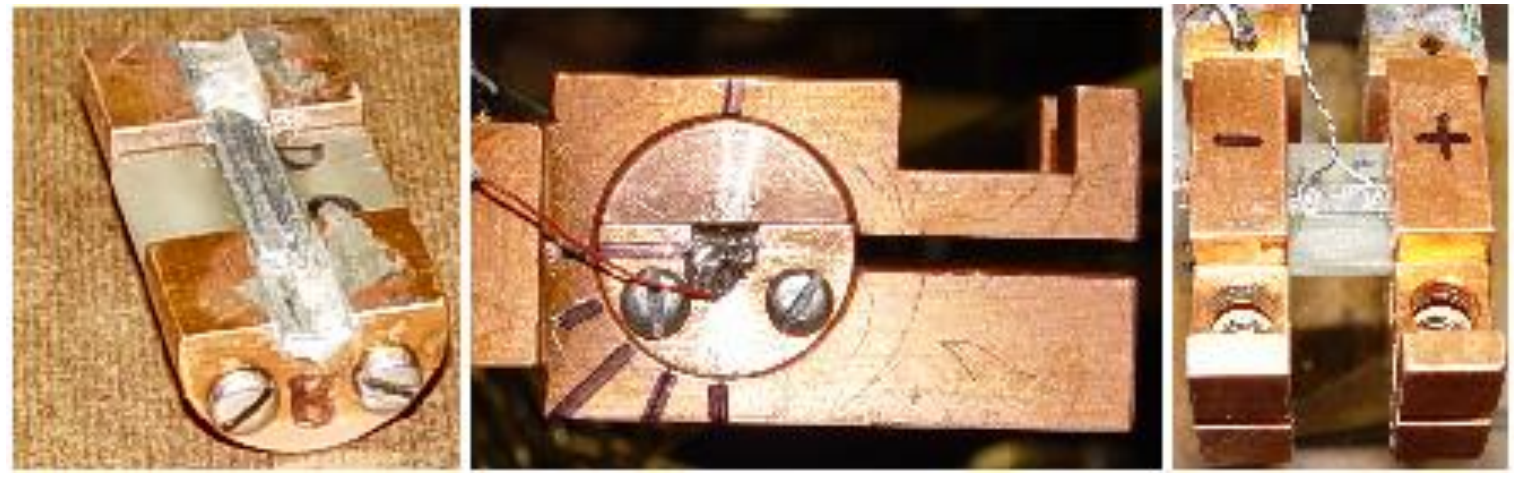

Figure 6.5.1. Sample holder (left), sample holder within the probe copper lugs (center), and instrumented sample (right). 
Samples were $38 \mathrm{~mm}$ long. Figure 6.5.1 shows the sample holder that was designed and used to change the direction of the c-axis of the tape with respect to the external magnetic field. The sample, supported in its middle part by G-10, was soldered within a groove on two $\mathrm{Cu}$ half cylinders (Figure 6.5.1, left). A splice length of $12 \mathrm{~mm}$ was used on each side of the sample to control contact resistance and heating power. The sample holder was placed at the desired angle within cylindrical holes in the probe $\mathrm{Cu}$ lugs (Figure 6.5.1, center). To provide electrical contact the lugs were then tightened using stainless steel screws. Current transfer length requirements determined the distance between the voltage taps, which was of $10 \mathrm{~mm}$ (Figure 6.5.1, right).

$\mathrm{I}_{\mathrm{c}}$ tests were performed as a function of magnetic field for the BSCCO-2223 tape, in the parallel and transverse field configurations, from $1.8 \mathrm{~K}$ to $33 \mathrm{~K}$. The $\mathrm{I}_{\mathrm{c}}$ performance at $1.8 \mathrm{~K}$ was more than $10 \%$ better up to $15 \mathrm{~T}$. It is also worth noting that these hermetic tapes were able to perform without any degradation after several thermal cycles.

To gauge field and temperature dependence of the anisotropy, we define the ratio of $\mathrm{I}_{\mathrm{c}}$, normalized to $I_{c}(77 \mathrm{~K}, 0 \mathrm{~T})$, in a parallel field to that in transverse field. This ratio was measured as a function of the applied magnetic field for various temperatures. The field dependence is consistent with being linear, with a slope that increases with temperature. Figures 6.5.2 and 6.5.3 show the angular dependence. The $I_{c}$ normalized to $I_{c}(77 \mathrm{~K}, 0 \mathrm{~T})$ as a function of the field angle at various magnetic fields is plotted at $4.2 \mathrm{~K}$ and $33 \mathrm{~K}$. The data taken at $0 \mathrm{~T}$ field are shown as representative of the measurement uncertainty. Most of the $I_{c}$ reduction occurs between 90 and 45 degree.
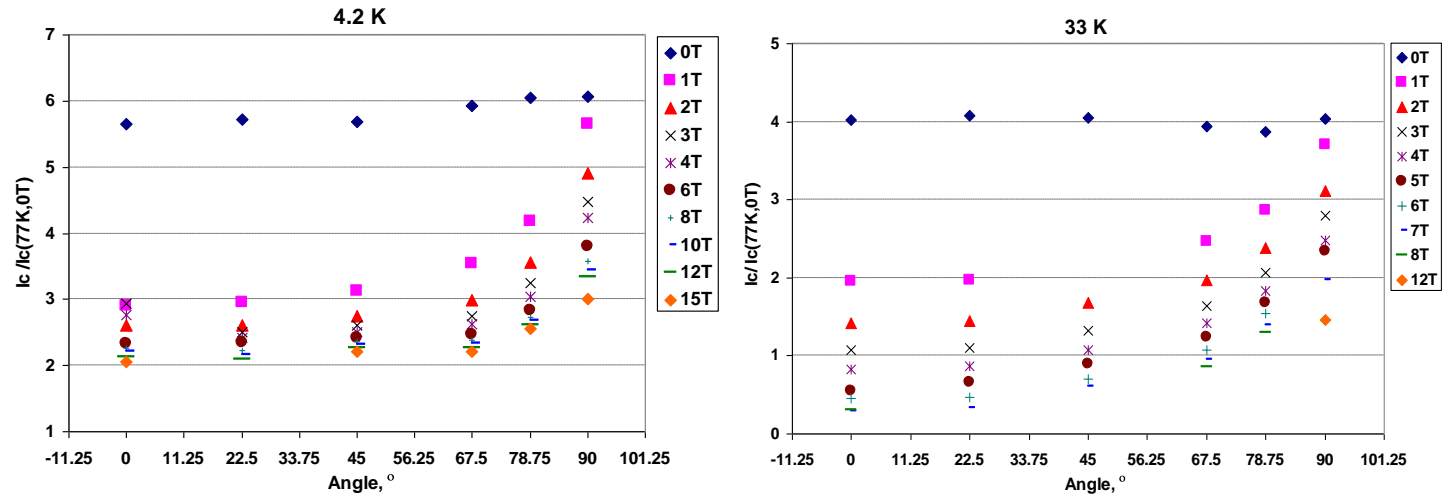

Figure 6.5.2. Normalized $I_{c}(4.2 \mathrm{~K})$ of the Bi-2223 hermetic conductor versus $B$ and $\Theta$.

Figure 6.5.3. Normalized $I_{c}(33 \mathrm{~K})$ of the Bi2223 hermetic conductor versus $B$ and $\Theta$.

Figures 6.5.4 and 6.5.5 show the $I_{c}$ normalized to $I_{c}(77 \mathrm{~K}, 0 \mathrm{~T})$ for $2 \mathrm{G} 348$ coated conductor, as a function of field and field angle at $4.2 \mathrm{~K}$ and $33 \mathrm{~K}$. Also for the $2 \mathrm{G}$, most of the $\mathrm{I}_{\mathrm{c}}$ reduction occurs between 90 and 45 degree, with a possible indication of a more gradual reduction with angle at $4.2 \mathrm{~K}$, but more data would be needed to confirm this. 


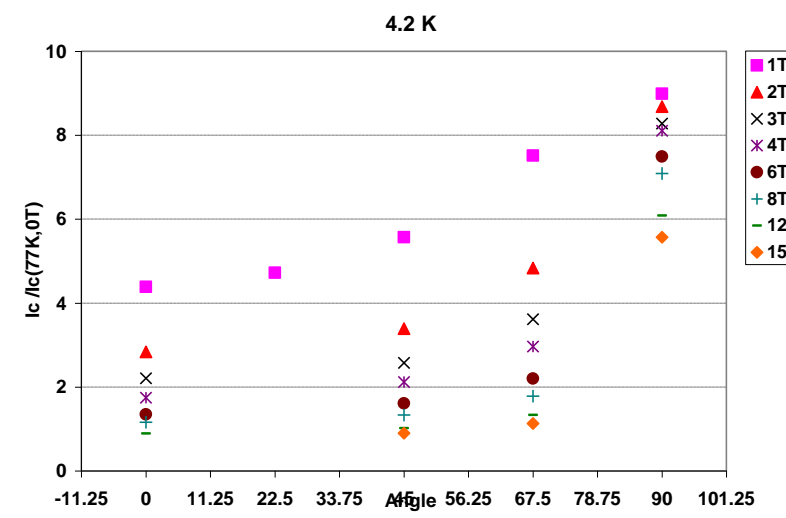

Figure 6.5.4. Normalized $I_{c}(4.2 \mathrm{~K})$ of the $2 G$ coated conductor versus $B$ and $\Theta$.

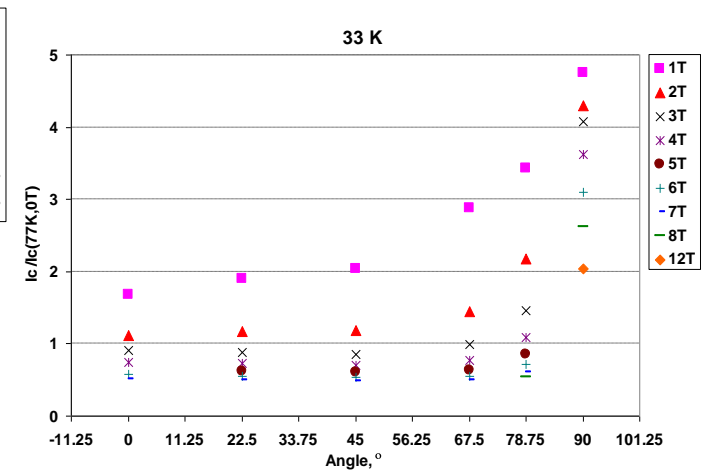

Figure 6.5.5. Normalized $I_{c}(33 \mathrm{~K})$ of the $2 G$ coated conductor versus $B$ and $\Theta$.

\subsection{Comparison with Low Temperature Superconductors}

Figure 6.6.1 shows the engineering critical current $\mathrm{J}_{\mathrm{E}}$ at $4.2 \mathrm{~K}$ and magnetic fields up to $15 \mathrm{~T}$, for $2 \mathrm{G} 348$ and $\mathrm{Bi}-2223$ superconductors, and round $\mathrm{Bi}-2212(\mathrm{OST}), \mathrm{Nb}_{3} \mathrm{Sn}$ and $\mathrm{NbTi}$ wires. For the calculation of $\mathrm{J}_{\mathrm{E}}$ of the $2 \mathrm{G} 348$ conductor the entire cross section was used. Below $15 \mathrm{~T}$, the $\mathrm{J}_{\mathrm{E}}$ of the RPP $\mathrm{Nb}_{3} \mathrm{Sn}$ wire exceeds that of all HTS at $4.2 \mathrm{~K}$. Above $17 \mathrm{~T}$ the Bi-2212 conductor shows an excellent overall performance. The 348 superconductors show a much higher current density in parallel fields, indicating excellent potential for high current density insert magnets for very high fields. For complete solenoids in which end sections operate with high perpendicular field components the $\mathrm{B}_{\text {perp }}$ performance is currently too low and needs further pinning enhancement for this field direction. The Bi-2223 conductor shows a lower engineering critical current density than either Bi-2212 or 348, but combines high strength, react and wind capability, and low angular field dependence of $\mathrm{J}_{\mathrm{c}}$.

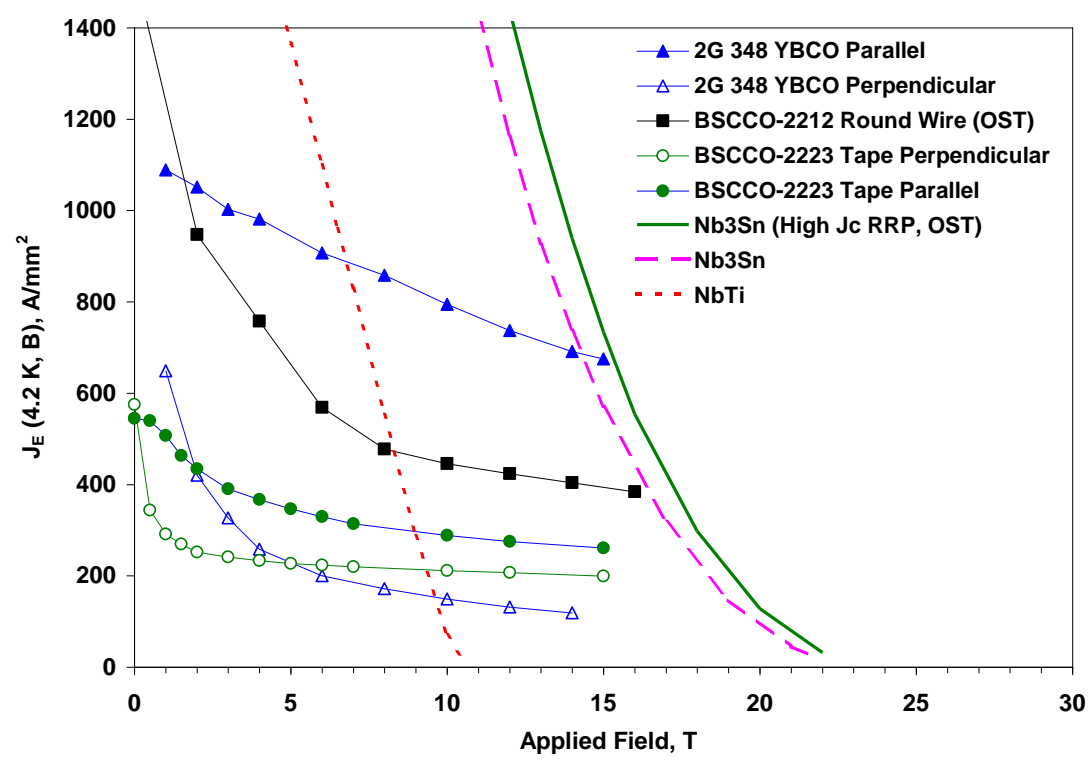

Figure 6.6.1. $J_{E}(B)$ at $4.2 \mathrm{~K}$ for 348 superconductors, Bi-2223 wire, round Bi-2212 wire (OST), and high current density $\mathrm{Nb}_{3} \mathrm{Sn}$ wire. 


\subsection{HTS material conclusions}

A major challenge for BSCCO-2212 are the powder leaks when heat treating cable. Given the excellent performance of the Bi-2212 OST strands after cabling, it is worthwhile to devote future resources to solve this cable heat treatment problem. A sample holder was designed to perform $\mathrm{I}_{\mathrm{c}}$ measurements of HTS tapes under externally applied magnetic fields, with orientations varying from zero to 90 degree with respect to the c-axis. This was performed from $1.8 \mathrm{~K}$ to $33 \mathrm{~K}$ up to $15 \mathrm{~T}$, while providing up to 1200 A of current. The data are consistent with similar measurements performed by AMSC in a perpendicular field at $\left.4.2 \mathrm{~K}^{35}\right]$. The engineering critical current for the new double layer hybrid 2G HTS superconductor competes well with existing conductors for parallel field orientations. With further c-axis pinning development, it is an excellent candidate for high field insert magnets operating above 16-18 T. For the design of high field solenoids, measurements at $4.2 \mathrm{~K}$ and at fields greater than $15 \mathrm{~T}$ should be addressed for the future, as well as the axial strain dependence.

\section{R\&D plan and goals}

It is anticipated that, by about 2012, there will be (a) initial results from the LHC, (b) a clarification of the ILC timeline, and (c) some sort of initial design report for a multi-TeV electron-positron collider (CLIC). It is desirable that, on this same timescale, Muon Collider R\&D progresses far enough for the community to be able to judge whether this new type of lepton collider is a viable option for the long-term future. Examples of the basic questions that we would like to address by 2012 are:

- Do high-pressure RF cavities work in a magnetic field with beam ?

- Can RF be integrated into a HCC without significantly degrading the cooling performance?

- Can a very long high-pressure hydrogen channel be safely operated, and will it need intermediate pressure windows ?

- Is there a solution for high gradient RF operating in appropriate magnetic fields for the phase rotation and Guggenheim-type cooling channels ?

- Can liquid hydrogen wedge absorbers and LiH absorbers be safely built and operated ?

- Is there a viable Parametric Ionization Cooling channel design with corrected chromatic and spherical aberrations?

- What is the maximum field that is plausible for an HTS solenoid cooling channel, how can quenches be handled, how can the magnet be fabricated, and is the technology radiation hard?

- How many muon bunches per cycle is optimum for a Muon Collider, and what minimum $\beta^{*}$ is achievable ? 
- How far in the forward direction can the detector operate, given the expected backgrounds?

- What collider magnet design can handle the decay electrons, and what do the beam collimators and radiation handling systems look like?

In the coming year, following the first year of MCTF activities, we intend to compile a complete list of the R\&D questions that we believe can be answered in the next 5 years with an appropriate $\mathrm{R} \& \mathrm{D}$ program. It is desirable that this important activity is pursued in close collaboration with the NFMCC. It is intended that this will lead us towards a 5 year R\&D plan that will employ the available Muon Collider R\&D support to address the most important open questions, and put us in a position to conduct an end-to-end Muon Collider simulation towards the end of this 5 year period. In the last 12 months an initial list of MCTF R\&D questions, focused on the cooling channel and collider ring, has already emerged, and provides the basis for the FY08 R\&D plan.

\subsection{FY08 R\&D Plan}

The main MCTF FY08 activities include (i) in collaboration with the NFMCC, compiling a complete list of R\&D questions to be addressed in the next 5 years, (ii) continued work on Muon Collider ring and cooling channel design and simulations, (iii) support of the continued NFMCC RF R\&D activities in the MTA, (iv) bringing beam to the MTA and supporting the first Muons Inc. HPRF cavity beam test, (v) construction of a four-coil HCC magnet prototype, (iv) continued HTS conductor studies and working towards a national HTS magnet collaboration, (v) continued studies of the options for a $6 \mathrm{D}$ cooling experiment and the development of HCC cooling channel components.

\subsubsection{Design and simulations}

The collider ring studies ultimately will define the required cooling channel performance. At present there are many open questions that must be addressed before the optimum collider design becomes clear. In FY08 the areas of study include:

- study of implications of the "dipole first" option for detector protection;

- feasibility study of electrostatic orbit separation for multi-bunch operation

- beam-beam simulations;

- detailing of the design with corrector circuits, injection and collimation systems;

- studying possible solutions for a low emittance collider ring.

In addition to the collider ring studies, there are many 6D cooling channel design questions that must be addressed. The areas of focus in FY08 include:

- more realistic modeling of the magnetic field for Guggenheim RFOFO channels;

- alternative Guggenheim design incorporating open cell RF cavities with solenoids in the irises;

- design of RF structure which can fit inside the helical solenoid;

- design and simulation of a segmented HCC channel;

- tracking simulations and optimization of a "FOFO snake";

- simulation of a 50T solenoid channel with required matching between the solenoids; 
- simulation of a channel incorporating Fernow's lattice with zero magnetic field at the RF;

- feasibility study of the PIC/REMEX scheme;

- more realistic modeling of the bunch merging process at initial stages of $6 \mathrm{D}$ cooling;

- alternative scheme with bunch coalescing at high energy $(\sim 30 \mathrm{GeV})$.

\subsubsection{High Pressure RF Beam Test and the MUCOOL MTA RF R\&D}

A very pressing question to answer is: What happens to pressurized RF cavities in the presence of an intense beam? Are cooling channel designs using high-pressure RF cavities viable? The first beam test of the Muons Inc HPRF cell will begin to address this question. Supporting this test is our highest hardware R\&D priority for FY08. This requires finishing the proton beamline to the MTA, which will require a number of accesses to the LINAC tunnel. The waveguides carrying RF power to the MTA must also be rerouted and additional shielding added before the enclosure is ready to accept beam. It is expected that the beamline will be ready for commissioning in the spring, and that the first cavity test will happen soon thereafter. For the initial test, it is foreseen to use the existing Muons Inc button cavity. This should give an indication of the scale of any effect. Depending on the outcome, more detailed studies will follow using purpose-build cavities. Although the HPRF test is our highest priority, we will continue to support, also with high priority, the continued MUCOOL RF tests in the MTA. The vacuum cavity program will be extended to study various configurations of $\mathrm{E}$ and $\mathrm{B}$ field directions, possibly using a rotatable cavity. Furthermore, a configuration change is foreseen to elevate the magnets and RF cavities to beam height, followed by the installation of a transfer line to the cryo refrigeration plant. Testing the existing $201 \mathrm{MHz}$ cavity in a new solenoid is also considered a priority activity, but the new coil will probably not be available until FY09. The planning requires coordination between the HPRF preparations and the ongoing MUCOOL test program.

\subsubsection{Helical Cooling Channel magnet}

The Helical Cooling Channel magnet design work will continue in FY08, with a focus on the helical solenoid concept. A key deliverable will be the design, construction and test of a four-coil subscale section of helical solenoid in a vertical Dewar at the Fermilab Technical Division. This test will give valuable input to the magnet design work. It is foreseen to have a design review in the late fall of '08, build the magnet by the summer ' 08 and test it towards the end of the fiscal year. In addition to the four-coil test, a key design issue to be tackled this year is how to incorporate RF cavities into the helical magnet. Three conceptual solutions exists, but input from both magnet, cryo and RF engineers is needed to determine which solution is the most promising. One issue to be resolved is the lack of an available cryo engineer in the Technical Division magnet group. Other things to be studied include quench protection, power leads, powering schemes, cryostat and cooling technology (e.g. whether cryo-coolers can be used). A system for field measurements inside the magnet will likely have to be developed, since there is no free line-of-sight thru the helical bore. 


\subsubsection{High field solenoid/HTS}

At least in some scenarios, the final section of the cooling channel consists of very high field ( 50T) solenoids. A pulsed hybrid (part superconducting, part normal-conducting) solenoid of comparable strength is already operating at the National High Magnetic Field Laboratory in Tallahassee, FL, so it could be argued that the technology already exists. However, the power consumption of this device is very high because of the heat dissipated in the normal conducting part. It is hoped that the required power could be greatly reduced using High Temperature Superconductor. In addition, other cooling scenarios, such as the final part of the Helical Cooling Channel also require very high fields, which may be best implemented using High Temperature Superconductor technology. So far, the conductor industry has focused on HTS for the power distribution industry, which requires high current density at essentially zero magnetic field. This type of conductor is not suited for magnet construction. In order to create an incentive for the conductor companies to work on conductor useful for magnet design, the plan for the coming year is to form a collaboration of national institutions (similar to what exists for the $\mathrm{Nb3Sn}$ ), and pool resources for material purchase to a level that makes it interesting to the conductor companies. By also coordinating the testing of the materials, one can also avoid expensive duplication of test equipment. Ideally, such a collaboration, once in place, would be able to attract additional resources from the funding agencies. Discussions regarding such a collaboration include:

- Identify common ground for conductor needs

- Consolidate existing data on conductor tests

- Identify facilities and resources

- Making near and longer term plans for magnet-related conductor development and testing, magnet development

- Organize strategy for purchasing HTS conductor.

Locally at Fermilab, the plan for the coming year is continue testing on available HTS material, develop probes required to characterize conductor and close the literature "gap". One particular area of interest is tensile strain probes. A small oven in the Technical Division will be converted for HTS reaction, and a modest program to react strands, characterize temperature/time cycles, and compare results to strands reacted in industry, will be carried out. Material to support this activity on the out years will be acquired though the National Collaboration. Paper studies on high field solenoids and performance comparisons of the commercially available conductors will also continue.

\subsubsection{D Cooling experiment and HCC Component Development}

The prospects for a muon beam test of a $6 \mathrm{D}$ cooling channel section will depend upon the available $M \& S$ in the coming years. The experiment is likely to require approaching, or exceeding, $10 \mathrm{M} \$$ of $\mathrm{M} \& \mathrm{~S}$, and take a few years to prepare. Eventually, testing a real 6Dcooling channel prototype in a muon beam will be a necessary step in building community confidence in the technology and simulations. However, we must balance this need with the additional need of developing and bench testing viable technology that can be used for a $6 \mathrm{D}$ cooling channel. A generic demonstration of $6 \mathrm{D}$ cooling will not be useful unless we also have the technical know-how to build a real cooling channel. The ideal situation would be to develop realistic technology and test a cooling section built from this technology in a beam. To understand whether this is a sensible path to follow, 
given realistic expectations for the level of support in the coming years, we must identify the most promising technical solution for a 6D cooling channel, and estimate the costs of prototyping a short cooling channel section, preparing an appropriate beamline, experimental hall, utilities, and instrumentation. We plan to make these cost estimates during FY08 so that, at the end of the year, we will be able to decide on the best plan. At that time we expect to also have results from the high-pressure RF cavity beam test, and from the four-coil HCC magnet test.

\section{References}

${ }^{1}$ C. Ankenbrandt et al, Muon Collider Advanced Accelerator R\&D Proposal, Fermilab, 2006

${ }^{2}$ R.Palmer et al, "A Complete Scheme of Ionization Cooling for a Muon Collider", PAC07, Albuqueraue, NM.

${ }^{3}$ R.Johnson, Y. Derbenev, "Low emittance muon colliders", PAC07, Albuquerque, NM.

4 “ $\mu+\mu$ - collider - A feasibility study", BNL-52503, Fermi Lab Conf 96/092, LBNL-39046, 1996.

5 A. Garren and C. Johnstone, "A Ring Lattice for a 2-TeV Muon Collider”, P. 411-413, Proc. PAC 97, Vancouver.

${ }^{6}$ J. S. Berg et al., Phys. Rev. ST Accel. Beams 9, 011001 (2006).

${ }^{7}$ R. Palmer et al., Phys. Rev. ST Accel. Beams 8, 061003 (2005).

${ }^{8}$ A.Bogacz, PIC/REMEX Workshop, Fermilab, May 21-22, 2007

${ }^{9}$ Y.Derbenev "Ionization Cooling on Spiral Orbit", MUCOOL Note 108, 2000

${ }^{10}$ V.Balbekov et al, "Geant4 simulation and theoretical studies of a helical cooling channel", PAC01, Chiacgo, IL.

${ }^{11}$ Y. Derbenev and R. P. Johnson; PRSTAB 8, 041002 (2005)

12 Y. Derbenev, R.P. Johnson, "Parametric-resonance ionization cooling and reverse emittance exchange for muon colliders", COOL05, Galena, IL, AIP Conf.Proc.821:420-426 (2006).

${ }^{13}$ D.B. Cline, A. Garren, Y. Fukui, H. Kirk, "Comparison of 6D Ring Cooler Schemes and Dipole Coooler for $\mu+\mu$-Collider Development", PAC07, Albuquerque NM.

${ }^{14}$ Y. Fukui, D.B. Cline, A. Garren, H. Kirk, "6 Dimensional Muon Phase Space Cooling By Using Curved Lithium Lenses,” PAC07, Albuquerque NM.

${ }^{15}$ R.P. Johnson et al, "Mark II High-Pressure RF Test Cell Measurements with Molybdenum Electrodes at Lab G”, MuCoolNote-285

16 R.P.Johnson et al, "High pressure, high gradient RF cavities for muon beam cooling, LINAC2004, Lubeck, Germany..

17 R.P.Johnson et al, "Recent innovations in muon beam cooling and prospects for muon colliders", PAC05, Knoxville, TN.

18 P.Hanlet et al, "High pressureRF cavities in magnetic fields", EPAC06, Edinburgh, UK

19 R.P.Johnson, "Low emittance muon colliders", PAC07, Albuquerque, NM.

20 "Beam-Cavity Interaction for High Pressure Hydrogen Gas Filled Cavities", A.V. Tollestrup, MuCoolNote-514

${ }^{21}$ Y. Derbenev and R.P. Johnson, PRSTAB 8, 041002 (2005)

${ }^{22}$ K.Yonehara et al, "Simulations of a gas-filled helical muon beam cooling channel", PAC05, Knoxville, TN.

${ }^{23} \mathrm{~K}$. Yonehara et al, "Simulations of MANX, a practical six dimensional muon beam cooling experiment", COOL05, Galena, IL, AIP Conf. Proc. 821, 458 (2006)

${ }^{24}$ K.Yonehara et al, "Studies of a gas-filled helical muon beam cooling channel", Edinburgh, UK.

${ }^{25}$ V. S. Kashikhin, et. al., "Magnets for the MANX 6-D cooling demonstration experiment", PAC07, Albuquerque NM.

${ }^{26}$ Vl. Kashikhin et al, "Superconducting Helical Solenoid Systems for Muon Cooling Experiment at Fermilab", Magnet Technology Conference 2008 (MT-20), Philadelphia PA.

${ }^{27}$ C. Ankenbrandt et al, "Delivering Muons to the MTA area", Fermilab Beamsdoc-2716

${ }^{28}$ A. Jansson et al, "A Muon Beam for Cooling Experiments", PAC'07, Albuquerque, NM.

${ }^{29}$ S.A. Kahn et al., "High Field Solenoid Magnets for Muon Cooling”, PAC07, Albuquerque NM.

${ }^{30}$ V.V. Kashikhin et al., "Study of High Field Superconducting Solenoinds for Muon Beam Cooling", Magnet Technology Conference 2008 (MT-20), Philadelphia PA. 
31 TD-07-018 Fermilab Technical Division Internal Note "Summary of HTS Work at TD", Emanuela Barzi et al.

${ }^{32}$ http://www.magnet.fsu.edu/mediacenter/news/pressreleases/2007august7.html, http://www.superpowerinc.com/20070807.aspx

${ }^{33}$ H. W. Weijers et al., Supercond. Sci. Technol. 17, 636 (2003).

${ }^{34}$ H. Miao, K.R. Marken, M. Meinesz, B. Czabaj and S. Hong, "Development of Round Multifilament Bi2212/Ag Wires for high Field Magnet Applications", IEEE Trans. Appl. Sup., vol. 15, p. 2554, 2005.

${ }^{35}$ C.L.H. Thieme, et al," Non-magnetic Substrates for Low Cost YBCO Coated Conductors", IEEE Trans. Appl. Superc. 11, p. 3329 (2001). 